\title{
ANGPTL3 deficiency alters the lipid profile and metabolism of cultured hepatocytes and human lipoproteins
}

\section{Ruhanen, Hanna}

2020-07

Ruhanen , H, Haridas , P A N , Minicocci , I Taskinen , J H , Palmas , F , di Costanzo , A , D'Erasmo , L, Metso , J , Partanen, J , Dalli , J , Zhou, Y, Arca , M , Jauhiainen, M , Käkelä , R \& Olkkonen , V M 2020 , ' ANGPTL3 deficiency alters the lipid profile and metabolism of cultured hepatocytes and human lipoproteins ', Biochimica and Biophysica Acta. Molecular and Cell Biology of Lipids, vol. 1865 , no. 7 , 158679 . https://doi.org/10.1016/j.bbalip.2020.158679

http://hdl.handle.net/10138/332411

https://doi.org/10.1016/j.bbalip.2020.158679

cc_by_nc_nd

acceptedVersion

Downloaded from Helda, University of Helsinki institutional repository.

This is an electronic reprint of the original article.

This reprint may differ from the original in pagination and typographic detail.

Please cite the original version. 


\section{ANGPTL3 deficiency alters the lipid profile and metabolism of cultured hepatocytes and human lipoproteins}

Hanna Ruhanen ${ }^{1,2,3}$, Nidhina Haridas P.A. ${ }^{1}$, Ilenia Minicocci ${ }^{4}$, Juuso H. Taskinen ${ }^{1}$, Francesco Palmas ${ }^{5}$, Alessia di Costanzo ${ }^{4}$, Laura D’Erasmo ${ }^{4}$, Jari Metso ${ }^{1}$, Jennimari Partanen ${ }^{1}$, Jesmond Dalli, ${ }^{5,6}$, You Zhou ${ }^{7}$, Marcello Arca ${ }^{4}$, Matti Jauhiainen ${ }^{1}$, Reijo Käkelä ${ }^{2,3}$ \& Vesa M. Olkkonen ${ }^{1,8, *}$

${ }^{1}$ Minerva Foundation Institute for Medical Research, Helsinki, Finland; ${ }^{2}$ Molecular and Integrative Biosciences, University of Helsinki, Helsinki, Finland; ${ }^{3}$ Helsinki University Lipidomics Unit (HiLIPID), Helsinki Institute for Life Science (HiLIFE), Helsinki, Finland; ${ }^{4}$ Department of Translational and Precision Medicine, Sapienza University of Rome, Italy, ${ }^{5}$ Lipid Mediator Unit, William Harvey Research Institute, Barts and the London School of Medicine and Dentistry, Queen Mary University of London, London, United Kingdom; ${ }^{6}$ Centre for Inflammation and Therapeutic Innovation, Queen Mary University of London, London, UK;

${ }^{7}$ Systems Immunity University Research Institute and Division of Infection \& Immunity, Cardiff University, Cardiff, United Kingdom, ${ }^{8}$ Department of Anatomy, University of Helsinki, Finland.

${ }^{*}$ Corresponding author at: Minerva Foundation Institute for Medical Research, Biomedicum 2U, Tukholmankatu 8, FI-00290 Helsinki, Finland; Tel +358-2-94125705, e-mail vesa.olkkonen@helsinki.fi 


\section{ABSTRACT}

Loss-of-function (LOF) mutations in ANGPTL3, an inhibitor of lipoprotein lipase (LPL), cause a drastic reduction of serum lipoproteins and protect against the development of atherosclerotic cardiovascular disease. Therefore, ANGPTL3 is a promising therapy target. We characterized the impacts of ANGPTL3 depletion on the immortalized human hepatocyte $(\mathrm{IHH})$ transcriptome, lipidome and human plasma lipoprotein lipidome. The transcriptome of ANGPTL3 knock-down (KD) cells showed altered expression of several pathways related to lipid metabolism. Accordingly, ANGPTL3 depleted IHH displayed changes in cellular overall fatty acid (FA) composition and in the lipid species composition of several lipid classes, characterized by abundant $n-6$ and $n-3$ polyunsaturated FAs (PUFAs). This PUFA increase coincided with an elevation of lipid mediators, among which there were species relevant for resolution of inflammation, protection from lipotoxic and hypoxia-induced ER stress, hepatic steatosis and insulin resistance or for the recovery from cardiovascular events. Cholesterol esters were markedly reduced in ANGPTL3 KD $\mathrm{IHH}$, coinciding with suppression of the SOAT1 mRNA and protein. ANGPTL3 LOF caused alterations in plasma lipoprotein FA and lipid species composition. All lipoprotein fractions of the ANGPTL3 LOF subjects displayed a marked drop of 18:2n-6, while several highly unsaturated triacylglycerol (TAG) species were enriched. The present work reveals distinct impacts of ANGPTL3 depletion on the hepatocellular lipidome, transcriptome and lipid mediators, as well as on the lipidome of lipoproteins isolated from plasma of ANGPTL3deficient human subjects. It is important to consider these lipidomics and transcriptomics findings when targeting ANGPTL3 for therapy and translating it to the human context.

Keywords: Angiopoietin like 3, lipoprotein lipase, lipidomics, lipid metabolism, cholesterol ester, polyunsaturated fatty acid 


\section{Introduction}

Cardiovascular diseases (CVD) are one of the leading causes of death worldwide [1]. Dyslipidemia, mainly elevated low-density-lipoprotein (LDL) cholesterol and triglyceride rich lipoproteins (TRLs), is the major risk factor for the development of CVD. Statins and ezetimibe, which reduce LDL-cholesterol, are used as the most common standard therapeutics for CVD treatment [2]. However due to a high level of residual CVD risk refractory to these therapies, efforts have been made to identify novel targets and treatment modalities for CVD [3]. Angiopoietin like protein 3 (ANGPTL3) is one of the main targets in this line of research. Among the ANGPTL family of proteins ANGPTL3, 4 and 8, play crucial roles in lipoprotein metabolism. They are potent inhibitors of LPL, which hydrolyzes triglyceride rich lipoproteins (TRLs) [4;5]. Loss of function (LOF) mutations of the ANGPTL3 gene were initially found in human subjects with extremely low serum triglycerides [6] and were later found to confer protection from coronary artery disease [7]. The ANGPTL3 LOF variant carriers exhibit hypolipidemia and high insulin sensitivity [8;9]. They have low levels of all major plasma lipoprotein classes (VLDL, LDL and HDL) [8;9]. Accordingly, this disorder was designated as a familial combined hypolipidemia (FHBL2; OMIM \#605019) [6;8]. Moreover, plasma free fatty acid (free FA) level is reduced in the ANGPTL3 LOF carriers [9]. ANGPTL3 LOF variant carriers are not known to exhibit any adverse health effects and they seem to display an increased life expectancy [10;11]. ANGPTL3 deficient subjects have been reported to lack significant coronary atherosclerotic plaques and also heterozygous ANGPTL3 LOF variant carriers display 35$40 \%$ attenuated risk of CAD compared with the general population [7;12]. ANGPTL3 has therefore emerged as a potential therapeutic target for cardiovascular disorders. A human monoclonal antibody against ANGPTL3, REGN1500, was shown to lower plasma lipids in monkeys [13]. In addition, human subjects as well as mice treated with the anti-ANGPTL3 
antibody Evinacumab exhibited hypolipidemia [12]. Similar results were obtained in both humans and mice when ANGPTL3 was silenced using an antisense-oligonucleotide approach [14].

ANGPTL3 is a protein secreted by hepatocytes [15], and insulin downregulates its expression in liver and plasma [16]. Of note, ANGPTL3 acts as an inhibitor of both LPL and endothelial lipase (EL) [17;18]. Inactivation or suppression of ANGPTL3 reduces plasma lipids by enhancing LPL activity, reducing hepatic VLDL-triglyceride secretion and enhancing LDL/VLDL uptake by the liver [19;20]. The enhancement of EL activity upon ANGPTL3 inhibition may explain the reduction of HDL observed in ANGPTL3 LOF carriers [18]. ANGPTL3 was also reported to induce adipocyte lipolysis [21], which provides a putative explanation for the reduction of FAs in the plasma of the LOF carriers [9].

Even though the functions of ANGPTL3 in the circulation are relatively well characterized, many mechanistic questions regarding the molecular consequences of ANGPTL3 LOF and protection against CVD remain unanswered. Although recent metabolic signature of lipoprotein classes from ANGPTL3 deficient subjects based on NMR [22] introduced some novel observations, the detailed lipid composition of the plasma lipoproteins of these subjects and how it may be linked to CVD protection are unclear. Likewise, the intracellular role of ANGPTL3 as a regulator of the hepatic lipid composition and function have not been studied in detail. To aid in understanding the molecular mechanisms exerted by ANGPTL3 deficiency, we addressed the alterations of hepatocellular and plasma lipid molecular species profiles caused by ANGPTL3 LOF. 


\section{Material and Methods}

2.1 Cell culture and generation of immortalized human hepatocytes (IHHs) with ANGPTL3 stably knocked down

IHHs immortalized by SV40 large T-Antigen (IHH, ATCC® PTA-5565TM) were cultured in Williams E medium (Gibco by Life Technologies, 22551-022) with added 10\%(v/v) fetal bovine serum (FBS), $0.2 \mathrm{mg} / \mathrm{ml}$ glutamine, $100 \mu \mathrm{g} / \mathrm{ml}$ streptomycin and $100 \mathrm{U} / \mathrm{ml}$ penicillin. The cells were transduced with MISSION ${ }^{T M}$ shRNA (short hairpin RNA) Lentiviral Vector particles (TRCN0000242782, Sigma Aldrich) targeting ANGPTL3 (NM_014495.2), validated in Tikka et al. [23], or with a non-targeting shRNA (SHCOO2, Sigma Aldrich) [MOI (multiplicity of infection) 1]. Transduced cells were selected with $5 \mu \mathrm{g} / \mathrm{ml}$ puromycin and cultured further in the above medium containing puromycin.

\subsection{Gene expression analysis (qPCR)}

Expression of ANGPTL3 and SOAT1 genes were analyzed by quantitative polymerase chain reaction ( $q P C R$ ). Total RNA was extracted from the ANGPTL3 KD and control IHH cells using PureLink ${ }^{\circledR}$ RNA Mini Kit according to the manufacturer's protocol. cDNA synthesis was carried out using SuperScript® ${ }^{\circledR}$ ILO $^{\mathrm{TM}}$ reverse transcriptase kit (Invitrogen, 11754050). Quantitative real time PCR was performed with gene-specific primers (Supplementary table 1) and LightCyclerß 480 SYBR Green I Master mix (Roche, 04707516001) using Light Cycler 480 II instrument (Roche Applied Science, Penzberg, Germany). $\beta$-actin and RPLP0 (Large Ribosomal Protein) were used as housekeeping references to which the data were normalized. 


\subsection{Western blotting}

ANGPTL3 silenced IHH hepatocytes and control cells were lysed in RIPA buffer (15 mM Tris- $\mathrm{HCl}, \mathrm{pH} 7.4,1.25 \%$ sodium deoxycholate, 1\% NP40, 1\% SDS, 1 mM EDTA, $150 \mathrm{mM}$ $\mathrm{NaCl})$. Proteins were resolved on $12 \%$ sodium dodecyl sulfate polyacrylamide gels and blotted onto PVDF membrane using BioRad transblot system. Blots were probed with antiANGPTL3 (R332), an antibody developed in New Zealand White rabbits against a peptide EDQYKQLNQQHSQIKEIENQ of human ANGPTL3, or anti-SOAT1 (Santa Cruz sc69836), in Tris buffered saline containing 5\% bovine serum albumin and $0.1 \%$ Tween 20 . Enhanced chemiluminescence reagent (BioRad Clarity Max ${ }^{\mathrm{TM}}$ or Thermo Scientific, Waltham, MA) was used to develop and BioRad Chemidoc imaging system was used to capture the signals. Protein bands were normalized to the total protein content of the corresponding lane using Image Lab software (BioRad).

\subsection{Next generation RNA sequencing}

$\mathrm{IHH}$ cells were cultured on 6 -well plates $(n=4)$ in the conditions specified above for $48 \mathrm{~h}$, after which they were changed into fresh growth medium without puromycin and incubated for $4 \mathrm{~h}$. RNA was extracted from the ANGPTL3 KD and control IHH cells using RNeasy ${ }^{\circledR}$ Mini Kit (Qiagen, 74104) according to manufacturer's protocol.

NEBNext Ultra Directional RNA Library Prep Kit for Illumina was used to generate cDNA libraries for next generation sequencing. The mRNA was purified using magnetic beads and then fragmented in order to generate inserts of approximately $200 \mathrm{bp}$, and primed with random primers. The first strand cDNA synthesis utilized Actinomycin D, which inhibits the DNA polymerase activity of the reverse transcriptase increasing strand specificity. In the second strand cDNA synthesis dUTP labelled oligo nucleotides were incorporated to mark 
the second strand with uracils $(U)$. The cDNA synthesis product was purified with Agencourt AMPure XP beads. Next, the CDNA was end-repaired, and adapter ligated utilizing dA-tailing. The adaptor ligated DNA went through a bead-based size selection after which the final PCR enrichment took place. Each sample was given a unique index to enable pooling of multiple samples (multiplexing) for sequencing. During the high-fidelity PCR, USER (Uracil-specific Excision Reagent) enzyme cut away the uracil strand preserving only the first strand. In addition, the loop adaptor was cut open to enable the PCR. The amplified library was then purified using AMPure XP Beads. Library quality was assessed by Bioanalyzer (Agilent DNA High Sensitivity chip) and library quantity by Qubit (Invitrogen). Sequencing was performed with Illumina NextSeq system, High Output 75 cycles kit.

STAR 2.5.1b (PMID: 23104886) was used for aligning output sequencing data to human reference genome (GRCh38) and "featureCounts" program from the Subread 1.5.0 (PMID:24227677) to count uniquely mapped fragment against genomic features defined by the GENCODE annotation file (Homo_sapiens.GRCh38.89.gtf). Differential gene expression (PMID: 25516281) was analyzed with Deseq2 1.22.1 and Benjamini-Hochberg method was used to control for false discovery rate. The transcripts with no counts in all samples were removed. Ensembl version 98 was used for gene annotation.

Gene set enrichment analysis and gene set over-representation analysis were performed using the clusterProfiler R package [24]. Overrepresentation analysis was performed using all genes, which had an adjusted p-value $<0.05$ (Benjamini-Hochberg). Network graphs were produced using igraph [25] and the results were visualized using Cytoscape [26]. 


\subsection{Study participants and lipoprotein isolation from human plasma}

The clinical characteristics of the study participants are summarized in Supplementary table 2. The study protocol was approved by the Ethical Committee of Sapienza University of Rome, and informed consent was obtained from all the study participants. ANGPTL3 LOF homozygotes and controls were matched for age, sex and BMI. The participants selected for this study are a subpopulation of a larger cohort described in previous studies [22;27]; The groups have been reported to have comparable dietary intake, physical activity, smoking prevalence, and use of anti-inflammatory medications.

Lipoprotein fractions (VLDL, LDL and HDL) were isolated from plasma by sequential density ultracentrifugation as described previously [28].

\subsection{Lipidomics of IHH cells and lipoprotein fractions by ESI-MS/MS and gas chromatography (GC)}

For IHH lipidomics, ANGPTL3 KD and control IHH cells were first cultured on 6-well $(n=6)$ plates for $48 \mathrm{~h}$ as mentioned above. Fresh medium without puromycin was changed into the wells and cells were incubated for $24 \mathrm{~h}$, washed with ice-cold PBS and finally scraped into $1 \mathrm{ml}$ of ice-cold $0.25 \mathrm{M}$ sucrose. An aliquot of $100 \mu \mathrm{l}$ was taken from each sample for total protein analysis using BCA assay (Thermo Fisher Scientific, 23227). The samples were stored in $-80^{\circ} \mathrm{C}$ for 7 weeks before lipid extraction.

Lipids of IHH cell samples and lipoprotein fractions were extracted according to Folch at al. [29]. Solvents were evaporated and the lipid extracts immediately dissolved in chloroform/methanol 1:2 (by vol) and right before mass spectrometry $1 \% \mathrm{NH}_{4} \mathrm{OH}$ was added together with SPLASH® LIPIDOMIX® Mass Spec Standard (Avanti Polar Lipids, 330707). The samples were injected into the electrospray source of a triple quadrupole 
mass spectrometer (Agilent 6490 Triple Quad LC/MS with iFunnel Technology; Agilent Technologies, Santa Clara, CA) and their lipids species were identified and quantified using lipid class specific detection modes, as previously described [30]. Retrieved spectra were processed by MassHunter Workstation qualitative analysis software (Agilent Technologies, Inc.), and individual lipid species were quantified using the internal standards and LIMSA software [31].

An aliquot of the lipid extracts were used for determining the FA composition of total lipids of the samples by GC according to the principles described in Käkelä et al. [32]. The extracted lipids were transmethylated by heating with $1 \% \mathrm{H}_{2} \mathrm{SO}_{4}$ in methanol under nitrogen atmosphere, and the formed FA methyl esters (FAME) extracted twice with hexane, dried with anhydrous $\mathrm{Na}_{2} \mathrm{SO}_{4}$ and concentrated. The FA structures were identified using GCMS-QP2010 Ultra (Shimadzu Scientific Instruments, Kyoto, Japan) with mass selective detector (MSD) and the FA proportions quantified using Shimadzu GC-2010 Plus equipment with flame-ionization detector (FID). Both instruments were equipped with Zebron ZB-wax capillary columns $(30 \mathrm{~m}, 0.25 \mathrm{~mm}$ ID and film thickness $0.25 \mu \mathrm{m}$; Phenomenex, Torrence CA, USA).

\subsection{Metabolic labeling of cholesterol ester}

Control IHH and ANGPTL3 KD cells were grown on 6-well plates to confluency. Cells were then incubated with $\left[{ }^{3} \mathrm{H}\right]$ acetic acid $(50 \mu \mathrm{Ci} /$ well; Perkin Elmer, NET003025MC) in medium without puromycin for $3 \mathrm{~h}$, washed with cold PBS and scraped into cold $2 \% \mathrm{NaCl}$. Total lipids were extracted according to Bligh and Dyer [33]. The lipid classes of the samples were separated by thin layer chromatography on silica gels and hexane/diethyl ether/acetic acid $/ \mathrm{H}_{2} \mathrm{O}(65: 15: 1: 0.25, \mathrm{vol} / \mathrm{vol})$ as the solvent system. CE standard was run 
along with the samples, and lipid spots were stained with iodine vapor. CE bands were scraped and their $\left[{ }^{3} \mathrm{H}\right]$ radioactivity was measured by liquid scintillation counting, and the results normalized for total cell protein. Additionally, $\left[{ }^{3} \mathrm{H}\right]$ oleic acid labeling $(2.5 \mu \mathrm{Ci} /$ well; Perkin Elmer, NET289005MC) was carried out as described above for $\left[{ }^{3} \mathrm{H}\right]$ acetic acid.

\subsection{Lipid mediator analysis}

Control IHH and ANGPTL3 KD cells were cultured for $48 \mathrm{~h}$ on 6 -well plates $(n=3)$ in complete Williams E medium with puromycin after which the medium was changed to William's E medium (Gibco, A1217601, no phenol red) with added 10\% (v/v) FBS, 0.2 $\mathrm{mg} / \mathrm{ml}$ glutamine, $100 \mu \mathrm{g} / \mathrm{ml}$ streptomycin and $100 \mathrm{U} / \mathrm{ml}$ penicillin. After $24 \mathrm{~h}$ incubation the medium was changed to one containing $1 \%$ FBS. After $1 \mathrm{~h}$ the plates were frozen and stored in $-80^{\circ} \mathrm{C}$ before analysis. Also a control sample without cells was prepared simultaneously using the same conditions.

Subsequently, two volumes of cold $\mathrm{MeOH}$ containing deuterium-labelled synthetic $\mathrm{d}_{4}$ PGE $2, d_{8}-5-H E T E, d_{4}-L T B_{4}, d_{5}-L X A_{4}, d_{5}-R v D 2, d_{5}-L_{T C}, d_{5}-L^{2} D_{4}, d_{5}-L_{T E}$ (500 pg each), $d_{5}-M a R 1, d_{5}-M a R 2, d_{5}-R v D 3$ (250 pg each), $d_{5}-\operatorname{RvE1}(100 \mathrm{pg})$, and $d_{5}-17 R-R v D 1$ (25 pg) were added onto the wells. Cells were lifted and transferred together with the supernatant. These were then stored at $-20^{\circ} \mathrm{C}$ for at least $45 \mathrm{~min}$ and then centrifuged at $2500 \mathrm{rpm}$ for $10 \mathrm{~min}$. Supernatant was collected and concentrated to $\sim 1.0 \mathrm{ml}$ using a gentle stream of nitrogen gas (TurboVap LV system, Biotage). Solid phase extraction (SPE) was then performed through ExtraHera automated extraction system (Biotage) adding $9 \mathrm{ml}$ of aqueous pH 3.5 HCl solution. The acidified samples were then loaded onto conditioned C18 $500 \mathrm{mg}$ 200-0050-B cartridges (Biotage). Samples were washed with $4.0 \mathrm{ml}$ of $\mathrm{H}_{2} \mathrm{O}$ and $5.0 \mathrm{ml}$ of hexane, and products eluted using $4.0 \mathrm{ml}$ of methyl formate. Solvent was 
evaporated using a gentle stream of nitrogen (TurboVap LV, Biotage) and samples resuspended in $40 \mu \mathrm{l}$ of $\mathrm{MeOH}: \mathrm{H}_{2} \mathrm{O}(1: 1, \mathrm{vol} / \mathrm{vol})$ solution. Samples were centrifuged at 2500 rpm for $5 \mathrm{~min}$ and the supernatant centrifuged again at $9900 \mathrm{rpm}$ for $10 \mathrm{~s}, 4^{\circ} \mathrm{C}$ [34].

LC-MS-MS profiling was performed as described previously [34]. Following C18 SPE and concentration, samples were analyzed using QTrap 6500+ (ABSciex) MS system, coupled with a Shimadzu SIL-20AC HT auto sampler, and LC-20AD LC pumps. Agilent C18 Poroshell column $(150 \mathrm{~mm} \times 4.6 \mathrm{~mm} \times 2.7 \mu \mathrm{m})$ was used to separate lipid mediators. Using a constant flow rate of $0.5 \mathrm{ml} / \mathrm{min}$, eluents gradient started at 20:80:0.01 (vol/vol/vol) in $\mathrm{MeOH} / \mathrm{H}_{2} \mathrm{O} /$ acetic acid for $0.2 \mathrm{~min}$ that was ramped to 50:50:0.01 ( $\mathrm{vol} / \mathrm{vol} / \mathrm{vol}$ ) over $12 \mathrm{~s}$, maintained for $2 \mathrm{~min}$, ramped to 80:20:0.01 ( $\mathrm{vol} / \mathrm{vol} / \mathrm{vol}$ ) over $9 \mathrm{~min}$ and maintained for 3.5 $\min$, then ramped to $98: 2: 0.01(\mathrm{vol} / \mathrm{vol} / \mathrm{vol})$ and maintained for $5.5 \mathrm{~min}$. Mediators were identified by matching retention time to synthetic and authentic materials using a MRM developed with signature parent ion and characteristic daughter ions coupled with an Enhanced Product Ion (EPI) for matching mass spectra. Quantification was obtained through calibration curves from synthetic or authentic compounds and deuterium-labeled lipid mediators.

\subsection{Statistical analysis}

For univariate comparisons statistical differences were tested using a two-tailed Student's t-test. Principal Component Analysis (PCA) (Sirius, PRS, Bergen, Norway) was applied for multivariate comparisons of detailed lipid profiles. PCA reveals compositional differences between the samples, and also shows the lipid species mainly responsible for the variation in the data. Data used for the PCA were arcsine transformed to improve data normality and biplots showing the relative positions of the samples and variables were created using 
the first two principal components. The differences between the groups seen in the PCA were further quantitatively analyzed using Soft Independent Modeling of Class Analogy (SIMCA; Sirius) [35]. For lipid mediator statistics multivariate Partial Least Square Discriminant Analysis (PLS-DA) was performed by means of the software SIMCA (Umetrics), building a model with $\mathrm{R} 2 \mathrm{X}=1, \mathrm{R} 2 \mathrm{Y}=1$, and $\mathrm{Q} 2=1$. Variable Importance in Projection (VIP) score was used to highlight the most important lipid mediators discriminating the two groups.

\section{RESULTS AND DISCUSSION}

\subsection{Several genetic pathways related to lipid metabolism are altered in ANGPTL3 knock-down hepatocytes}

ANGPTL3 KD hepatocytes $(\mathrm{IHH})$ were created using an shRNA lentiviral vector. An approximately $90 \% \mathrm{KD}$ efficiency was observed at mRNA level when compared to control cells transduced with non-targeting shRNA (Figure 1A), and the KD effect was evident also at the protein level (Figure 1 B and C). KEGG pathway analysis of the next generation RNA sequencing (RNAseq) data of the ANGPTL3 KD and control IHH cells revealed that several pathways related to lipid metabolism are affected upon the knockdown (Table 1). Similarly, when Reactome pathway analysis was performed using only the statistically significantly up/downregulated genes, all of the ten most affected pathways in the ANGPTL3 KD cells were related to lipid and FA metabolism (Supplementary Figure 1). Thus we chose to study the cells further using lipidomics approaches to address hepatocyte lipid composition and metabolism. 
When looking at the other pathways significantly affected in the KEGG enrichment analysis, two pathways related to longevity emerged and were among the 50 most altered pathways: Longevity regulating pathway - multiple species, and Longevity regulating pathway (Supplementary table 3). This is an interesting and relevant observation when considering that subjects homozygous for LOF variants of ANGPTL3 reach older age than the average population [11]. The Campodimele region, where the ANGPTL3 loss-offunction mutations are found enriched, is known for the longevity of its residents, with the town's population now expected to live to an average age of $95[10 ; 36]$. Moreover, insulin sensitivity is increased in the ANGPTL3 LOF subjects [9]. According to the present KEGG enrichment analysis, also pathways related to insulin resistance/sensitivity, signaling and secretion are altered in the ANGPTL3 KD cells. In a previous study by Tikka et al. [23], it was reported that the KD of ANGPTL3 in IHH improves glucose uptake and that during insulin stimulation the cells secrete TAG-poor VLDL instead of TAG-enriched large VLDL particles. They concluded that silencing of ANGPTL3 improves insulin sensitivity of the $\mathrm{IHH}$ and suggested a liver-specific mechanism to be involved in the insulin-sensitive phenotype of ANGPTL3 LOF subjects. The present RNAseq observations support this notion.

\subsection{The ANGPTL3 knock-down cells are enriched in n-3 and n-6 polyunsaturated and depleted in monounsaturated fatty acids}

As the first lipidomic approach we determined the total FA profile of ANGPTL3 KD and control IHHs by GC. The reported profile represents FAs derived from all lipid species of the cell and the results are presented as molar percentages (Supplementary table 4). In ANGPTL3 KD cells the sum of monounsaturated FAs (MUFAs) was significantly decreased and the sums of both $n-3$ and n-6 polyunsaturated fatty acids (PUFAs) were 
increased when compared to controls (Figure 2A). However, there was no difference in the $n-3 / n-6$ FA ratio or the unsaturated/saturated FA ratio between control and ANGPTL3 KD cells. When the FA data were subjected to principal component analysis (PCA) (Figure 2B), the difference between control and ANGPTL3 KD cell groups reached the statistical significance when tested with SIMCA $(p<0.05)$. The groups separated from each other in the direction of principal component 2 (PC 2), which explained $25.3 \%$ of the total variation. The FAs influencing PC2 the most were 20:5n-3 (eicosapentaenoic acid, EPA) and 20:4n-6 (arachidonic acid, AA) furthest from the origin in the upward direction, and relatively enriched in the ANGPTL3 KD cells, and 20:3n-9 (eicosatrienoic acid) furthest from the origin in the downward direction, and enriched in the controls. EPA and AA can be produced from essential FAs 18:3n-3 (a-linolenic acid) and 18:2n-6 (linoleic acid) via desaturation/elongation processes, respectively, or taken up from the culture medium. FA 20:3n-9 is considered a marker of essential fatty acid deficiency: It is a PUFA synthesized from the non-essential 18:1n-9 (oleic acid) [37]. Of note, we observed in the RNAseq an upregulation of CD36, FABP3, FABP5 and FABP6 mRNAs in the ANGPTL3 KD cells. Since FABP3, -5 and -6 facilitate the cellular uptake of long chain FAs and PUFAs [38-41], we consider it possible that the uptake of FAs and especially PUFAs from the culture medium is enhanced in the ANGPTL3 KD cells.

We next analyzed the IHH lipids at the class level (Supplementary tables 5-8) by mass spectrometry. There were no differences between the control and ANGPTL3 KD cells in the concentrations of the major phospholipid classes PC, PE and PI when normalized to total cellular protein (data not shown). PCA of lipid species profiles in these classes of lipids showed similar separation between control and ANGPTL3 KD cells largely driven by MUFA- (relative reduction in the KD cells) and PUFA- (enrichment in the KD cells) containing lipids as seen in the fatty acid profile (Supplementary Figure 2). PUFAs are 
released from glycerophospholipids through the action of phospholipases such as cytosolic PLA2 (cPLA2) [42;43], two isoforms of which, PLA2G4A and PLA2G4C, are upregulated in our RNAseq data. Cytosolic PLA2 shows specificity for PUFAs, especially for 20:4n-6, 22:6n-3 (docosahexaenoic acid, DHA) and 20:5n-3 [42;43], and plays an important role in the initiation of cellular signaling, hydrolyzing the ester bond at the sn-2 position of glycerophospholipids. Thereby the released PUFAs can be used for the synthesis of bioactive lipid mediators [44]. Phosphatidylinositol (PI) is considered a major source of $20: 4 n-6$ released by cPLA2 since $20: 4 n-6$ is the most abundant PUFA in this PL class, the largest PI component being the 20:4n-6-containing species $38: 4$ (m/z 885 [45;46]. Indeed, in the PCA of our data PI 38:4 was the variable responsible for the largest part of the data variation on PC1 in the leftward direction, and the high level of PI38:4 separated ANGPTL3 KD samples from the control cells. Similarily, the PI 38:5 (likely a mixture of FAs 20:4, 22:5, 20:5 in the sn-2 position) was a characteristic of the ANGPTL3 KD cells.

Interestingly, it has been established that the relative FA compositions of the PI-derived signaling mediators phosphatidylinositol-phosphates (PIPs) reflect that of PI [47] and that polyunsaturated diacylglycerol (DAG) species derived from $\mathrm{PI}(4,5) \mathrm{P}_{2}$ can activate the PKC $\alpha, \varepsilon$, and $\delta$ isoforms [46]. This may also in part explain why the PIP signaling KEGG pathways are significantly affected in the present transcriptomics data (Table 1): The increase of PUFAs in the ANGPTL3 KD cells may through this mechanism impact $\mathrm{PIP}_{2}$ signaling. Closer examination of the KEGG phosphatidylinositol signaling pathway (Supplementary Figure 3) shows that several of its genes are upregulated in the KD cells, but phospholipase C (PLC), a key component in the pathway, is strongly downregulated. This could represent an allostatic mechanism aimed at maintaining a balanced pathway activity. 


\subsection{Knocking down ANGPTL3 changes the lipid mediator profile of hepatocytes}

Prompted by analyses showing changes in several KEGG pathways related to FA and glycerophospholipid metabolism as well as the increase of PUFAs in the ANGPTL3 KD cells, we decided to explore the bioactive PUFA-derived lipid mediators produced by the cells (Supplementary table 9). The results shown represent the sum of intracellular and secreted lipid mediators since both the cells and the medium were analyzed as one sample. There was a clear trend towards an increase of lipid mediators in the ANGPTL3 KD cells, and PLS-DA analysis separated the ANGPTL3 KD and control samples (Figure 3A). Figure 3B displays VIP scores of the 15 most important lipid mediators affecting separation of the groups in the PLS-DA analysis. The mediator with the highest VIP score was RvD6, an important specialized pro-resolving mediator (SPM) with a suggested role in coronary artery disease (CAD). RvD6 was shown to be elevated in CAD patients receiving n-3 FA supplement and it promoted macrophage uptake of blood clots in vitro [48]. Maresin 2 (MaR2) and 22-OH-MaR1, which also showed high VIP scores, are SPMs characterized in macrophages [49] and neutrophils [50], respectively. Of note MaR1, the precursor to 22-OH-MaR1, protects hepatocytes from lipotoxic and hypoxia-induced ER stress [51] suggesting that $22-\mathrm{OH}-\mathrm{MaR} 1$ may display similar biological actions given that this further metabolite retains the potent biological actions of its parent SPM [50]. The protectin pathway products from both n-3 docosapentaenoic acid (DPA) and DHA, i.e. 10S,17S-diHDPA and 10S,17S-diHDHA (protectin DX), were also increased in ANGPTL3 KD cells. PDX prevents lipid-induced ER stress, thereby ameliorating hepatic steatosis and hepatic insulin resistance [52;53]. Another mediator with a high VIP score, 15-epi$\mathrm{LXA}_{4}$ is reported to down-regulate pro-inflammatory eicosanoids and potentiate the production of SPMs [54;55]. 
Prostaglandins (PG) $\mathrm{PGD}_{2}, \mathrm{PGF}_{2 \mathrm{a}}$ and $\mathrm{PGE}_{2}$ all had a high VIP score in the present data. They are generally referred to as pro-inflammatory mediators [56], but especially $\mathrm{PGE}_{2}$ is also important for the lipid mediator class switching at the beginning of the resolution phase as it decreases the production of pro-inflammatory leukotriene $B_{4}\left(L_{T B}\right)$ species [57]. The increased production of lipid mediators in the ANGPTL3 KD cells is consistent with the findings of Holopainen et al. [58], who concluded that elevated amounts of PUFAs (AA, EPA and DHA) in membrane phospholipids lead to increased production of lipid mediators. Increased substrate availability, PLA 2 upregulation, and also the substrate preference of $\mathrm{PLA}_{2}$ towards PUFA- containing phospholipids [42;43] provide plausible explanations for the observed increase of PUFA-derived lipid mediators in the ANGPTL3 KD cells.

\subsection{Cholesterol ester synthesis is reduced in ANGPTL3 KD cells}

In addition to elucidating the effects of ANGPTL3 KD on phospholipid and FA metabolism, we also investigated cholesterol metabolism by multiple approaches. First, we looked at the profile (Supplementary table 8) and total level (Figure 4A) of cholesterol esters (CEs) in the IHH by using ESI-MS/MS. The relative CE species profile of the ANGPLT3 KD cells showed similar alterations in the MUFA and PUFA-containing species as described for total cellular FAs and phospholipids (Figure 2; Supplementary tables 4-7). The level of the major CE species 18:1 was significantly $(p<0.001)$ reduced and that of the second largest component, the DHA-containing CE 22:6n-3, increased by $74 \%$. The latter finding appears as a class-specific change since DHA was not increased but rather decreased in the total IHH FA profile, driven by altered FA composition of glycerophospholipids. The total level of CE was significantly $(p<0.001)$ decreased in the ANGPTL3 KD cells when compared to controls (Figure 4A). Consistent with this finding, the expression of SOAT1, 
encoding ACAT1, an enzyme synthesizing CEs [59], was decreased in the KD cells both at the mRNA (Figure 4B) and protein (Figure 4C-D) levels. Accordingly, we next investigated CE synthesis and FA incorporation into CEs in the $\mathrm{IHH}$ by $\left[{ }^{3} \mathrm{H}\right]$ acetic acid and $\left[{ }^{3} \mathrm{H}\right]$ oleic acid labeling, respectively, and also analyzed the synthesized unesterified cholesterol in the $\left[{ }^{3} \mathrm{H}\right]$ acetic acid labeled samples. With both radioisotope precursors the level of labeled CEs was significantly $(p<0.001)$ reduced in the ANGPTL3 KD cells (Figure 4E-F), without a change in the labeling of unesterified cholesterol in cells treated with $\left[{ }^{3} \mathrm{H}\right]$ acetic acid (data not shown). Thus, knocking down ANGPTL3 in IHH significantly reduces the esterification of cholesterol. The reduction of CEs putatively mediated by suppression of ACAT1 function might affect the packaging of CEs to nascent VLDL and thereby their secretion. In agreement with these data we previously reported that there was a reduction of $\mathrm{CE} / \mathrm{apoB}$ ratio in plasma VLDL and $\mathrm{LDL}$ particles derived from ANGPTL3 LOF homozygote carriers compared with noncarrier subjects [9;22]. Decreases of VLDL and LDL were observed in pigs treated with avasimibe, an inhibitor of SOAT [60]. Consistently, overexpression of human SOAT1 and 2 in rat hepatoma McA-RH7777 cells was shown to increase CE synthesis and secretion [59]. Another inhibitor of SOATs, pactimibe sulfate, reduced plasma total cholesterol and stabilized atherosclerotic plaques in apolipoprotein E-deficient mice [61].

\subsection{ANGPTL3 deficiency alters the fatty acid profile of lipoproteins}

Since there is no indication of liver disease in ANGPTL3 LOF carriers [11], it is for ethical reasons impossible to obtain liver biopsies from these subjects. We were therefore unable to compare the human LOF mutation carriers' hepatic lipidome to that of the ANGPTL3 KD $\mathrm{IHH}$. However, we analyzed in detail their plasma lipoproteins, the composition of which is determined by both the physiology of hepatocytes secreting VLDL and HDL and the 
modifications mediated by plasma enzymes and lipid transfer proteins. We consequently studied how ANGPTL3 deficiency affects the lipid molecular species profile of lipoproteins, and whether any parallels can be found between the profiles of $\mathrm{IHH}$ subjected to ANGPTL3 KD and lipoproteins of subjects lacking ANGPTL3.

FA analysis of VLDL, LDL and HDL particles isolated from the plasma of ANGPTL3 LOF homozygotes $(n=5)$ and control subjects $(n=10)$ was performed by GC using the same approach as for the $\mathrm{IHH}$. Complete FA profiles of the lipoprotein fractions are found in Supplementary table 10. When inspecting individual FAs, the most prominent difference between the groups was a higher proportion of $18: 2 n-6$ in lipoproteins of the control subjects. The effect remained statistically significant in all lipoprotein fractions. In order to grasp the changes occurring in all the FAs and not merely in the largest components of the profile, we performed a PCA of the entire data using standardized mol\% values. A clear separation of the groups can be seen in the PCA biplot (Figure 5). PC1 explains $31.7 \%$ of the total variation and separates the different fractions (VLDL on the right, LDL in the middle and HDL on the left), whereas PC2 explains $21.3 \%$ of the variation and separates the control subjects (at the bottom) and the ANGPTL3 LOF homozygotes (at the top). The VLDL fractions contained relatively more MUFAs and FAs having <18-carbons, while the HDL fractions were enriched in 20-22-carbon PUFAs, >20-24-carbon SFAs or MUFAs (ANGPTL3 LOF homozygotes) and saturated dimethyl acetals derived from plasmalogens (controls). The right to left change (PC1) in the quality of the FAs is likely affected by the activity of LPL, which is higher in ANGPTL3 LOF homozygotes [9]. The efficiency of LPLmediated hydrolysis of ester bonds decreases with increasing chain length and unsaturation [62;63], and thus relatively short SFAs would be hydrolyzed first, followed by MUFAs, leaving the longest PUFAs to be hydrolyzed last. Importantly the FA profile of the different lipoprotein fractions is not influenced by the action of two lipid transfer proteins, 
CETP and PLTP, since no significant differences in their activities were observed between homozygous ANGPTL3 LOF variant carriers and control subjects [27]. We think that the impact of diet can be ruled out, as the comparison of dietary intakes between ANGPTL3 LOF subjects and controls did not reveal appreciable differences (data not shown).

\subsection{PUFA-containing TAGs are relatively increased in the lipoproteins of ANGPTL3 LOF homozygotes}

We next analyzed the lipid components of lipoprotein particles derived from ANGPTL3 LOF homozygotes and control subjects by ESI-MS/MS. The lipoproteins of the ANGPTL3 LOF homozygotes have been reported to contain less TAG and CEs when compared to those of control subjects [9]; Our results are consistent with these findings (fold changes of lipids at the class level are listed in Supplementary table 11 and species profiles of each class in Supplementary tables 12-16). At the species level the findings in CEs and TAGs were, however, opposite to each other. Compared to the controls, the CEs in the lipoproteins of ANGPTL3 LOF homozygous subjects included larger proportions of the species with 16:1 and 18:1 FAs (Supplementary table 13), whereas in TAGs the relative levels of PUFA-containing species were elevated (Figure 6, Supplementary table 12). The latter result is likely due to the increased activity of LPL in ANGPTL3 LOF homozygotes, since long PUFAs are poor substrates for LPL [62;63], and are thus retained. Interestingly, $\mathrm{Xu}$ et al. [20] found that knocking down ANGPTL3 in HuH7 cells increased the cellular concentration of long-chain TAGs, so it is possible that TAGs in VLDL particles of ANGPTL3-deficient subjects could be somewhat enriched in PUFAs already as they are secreted. The cellular concentrations of TAGs in the IHH model employed here were unfortunately too low to be reliably profiled at species level. Thus, we 
are unable to draw conclusions on the relationship of TAG molecular species composition in hepatocytes and plasma lipoproteins.

CEs are not substrates of LPL, since LPL is a serine-histidine hydrolase and its active site favors especially oxyester bond on the glycerol backbone [64-66]. Thus, CEs are not affected by the increased activity of LPL but another, unknown mechanism must modify their composition in the LOF mutation carriers. One putative explanation for these CE species shown in lipoproteins is altered substrate specificity of LCAT. Most of the CEs in

human plasma lipoproteins are derived from the function of LCAT [67]. Although LCAT displays specificity for the sn-2 position of PC, the composition of the CEs in human plasma does not match that of the sn-2 acyl group [68]. We can speculate that ANGPTL3 deficiency creates changes in FA distribution in PC molecules and therefore LCAT FA specificity or the positional specificity in PC [69] is altered and leads to generation of the CE species shown in the present study.

\subsection{ANGPTL3 deficiency changes the quality of SM and its ratio to PC}

The surface monolayer of lipoprotein particles consists mainly of PCs, sphingomyelins (SMs) and lysoPCs. We found intriguing changes between ANGPTL3 LOF subjects and controls in the species profiles of SM, PC and lysoPC in all the lipoprotein fractions (Figure 7A-C, Supplementary tables 14-16). SMs of the lipoproteins obtained from ANGPTL3 LOF homozygous subjects contained relatively more of the long SM species, especially $24: 1$ and $24: 2$, and less of short saturated SMs than the controls. The changes in lysoPCs were similar to those seen in TAGs (enrichment of PUFAs) and could be explained by the increased LPL-facilitated PLA1 activity shown to be able to release the 
FA in the sn-1 position of PC[70]. The study by McLean et al. [70] also indicated that the longer the FA in the sn-2 position, the higher activity LPL has against the sn-1 FA, resulting in lysoPCs enriched in sn-2 long chain FAs. Moreover, in the lipoproteins of ANGPLT3 LOF homozygous subjects there was an enrichment of alkyl ether PCs (Supplementary table 16). Since these species are synthesized in peroxisomes [71], the finding could imply that the peroxisomes of the LOF subjects are more active. However, to our knowledge nothing has been reported on the peroxisomal function in ANGPTL3 LOF mutation carriers. Another plausible explanation for the increase of alkyl ether PCs in the LOF lipoproteins is that LPL and EL, which are subject to regulation by ANGPTL3, are only hydrolyzing ester and not ether bonds [64;65;72]. Thereby, the elevated phopholipase activity of these enzymes in LOF subjects could result in a relative enrichment of nonsubstrate ether phospholipid species.

The SM/PC ratio was increased in all lipoprotein fractions derived from ANGPTL3 LOF homozygous subjects when compared to controls (Figure 7A). This could result from several different mechanisms. We did not see changes in the SM/PC ratio in the $\mathrm{IHH}$ cell model (data not shown). However, this does not rule out the possibility that nascent VLDLs may be enriched in SM; Once entering general circulation these particles could be rapidly lipolyzed by LPL, and the extra surface SM together with some PCs could be transported by PLTP to HDL and LDL, therefore increasing the proportion of SM in these particles. Indeed, it has been shown that SM is very efficiently transferred by PLTP [73].

The enrichment of SMs in lipoproteins obtained from ANGPTL3 LOF homozygotes is highly interesting in the light of the study of Ruuth et al. [74], where the saturated SM species and SM 16:1 were associated with LDL aggregation, which increases the risk of cardiovascular death. Thus, even though the SM/PC ratio is increased in the LDL of ANGPTL3 LOF homozygotes, the quality of SM (elevation of 24:1 and 24:2 species) in 
these subjects could make their LDL less prone to aggregation as compared to controls. It should also be noted that in HDLs an increased SM/PC ratio leads to an increased ability to receive cholesterol from cells, improving the cardioprotective capacity of the lipoprotein [75]. On the other hand, we have to consider that enrichment of HDL with SM inhibits cholesterol esterification, and this inhibition can be reversed by the degradation of SM, which demonstrates that $\mathrm{SM}$ is a physiological inhibitor of cholesterol esterification in circulation and competes with the PC acyl donor on the surface of HDL particle [76]. This would attenuate reverse cholesterol transport process which needs LCAT facilitated step of free cholesterol esterification. When interpreting the above data, one should also keep in mind that the absolute amounts of cholesterol and TAGs in the lipoproteins of ANGPTL3 deficient subjects are strongly reduced, which is by itself beneficial for the cardiovascular health of these individuals.

\section{CONCLUSIONS}

Loss-of-function mutations in ANGPTL3, an inhibitor of lipoprotein lipase (LPL), cause a drastic reduction of all major serum lipoproteins and are protective against the development of atherosclerotic CVD. Therefore, ANGPTL3 is emerging as a therapy target; Clinical trials with agents targeting ANGPTL3 have already reached phase 3. Although ANGPTL3 is produced almost exclusively by the liver, there is no data on its function in determining the hepatocellular gene expression patterns and lipidome. In the present study we characterized the impacts of ANGPTL3 depletion on the hepatocyte $(\mathrm{IHH})$ transcriptome and lipidome and whether there are parallels between the lipidomes of ANGPTL3-deficient hepatocytes and of plasma VLDL, LDL, and HDL isolated from homozygous ANGPTL3 LOF mutation carriers. 
$\mathrm{IHH}$ depleted of ANGPTL3 displayed distinct changes in total FA composition and in the molecular species composition of several lipid classes, characterized by an increase in the mol\% of $n-6$ and $n-3$ polyunsaturated FAs (PUFAs). The VLDL, LDL and HDL of the ANGPTL3 LOF subjects showed a significant drop of 18:2n-6, while several polyunsaturated TAG species were enriched, most likely due to elevated activity of LPL which prefers saturated and shorter chain FAs esterified to the glycerol backbone [62;63]. The LOF subjects' lipoproteins displayed an increased SM/PC molar ratio and enrichment of 24:1 and 24:2 SM species. Since the SM/PC ratio is increased in all lipoprotein fractions of $\mathrm{t}$ LOF subjects, it cardiometabolic implications are controversial. Similar to the $\mathrm{IHH}$, increased mol\% of PUFAs was found in LysoPCs, which could result from increased PLA1 activity of LPL [70].

Cholesterol esters were markedly reduced in the ANGPTL3 KD hepatocytes, coinciding with suppression of SOAT1/ACAT1. However, we did not see any change in the expression of SOAT2, which is the major isoform in human liver in vivo [77]. Previously, we have also detected a low CE/apoB100 molar ratio in the ANGPTL3 LOF homozygote subjects [9] as well as reduced CEs in VLDL remnants of these subjects [22]. However, the putative causality between the cultured hepatocyte and in vivo observations requires further investigation.

The PUFA increase in the ANGPTL3-depleted IHH coincided with an elevation of PUFAderived lipid mediators. Among these there were several mediators with documented functions in resolution of inflammation, protection from lipotoxic and hypoxia-induced ER stress, hepatic steatosis and insulin resistance $[52 ; 53]$ or in mediating the recovery from cardiovascular events [48;55]. Notably, the resolution of inflammation has emerged as a 
crucial physiological mechanism affecting atherogenesis [78-80]. Consistent with the lipid mediator data, altered expression of several KEGG pathways related to fatty acid metabolism was observed. The impact of ANGPTL3 inhibition on synthesis of the PUFAderived mediators deserves further investigation; when fresh plasma becomes available from the ANGPTL3 LOF carriers, it will be extremely interesting to study the lipid mediators in these subjects.

To conclude, the present work reveals distinct impacts of ANGPTL3 depletion on the hepatocellular lipidome, transcriptome and lipid mediators, as well as on the lipidome of lipoproteins isolated from the plasma of ANGPTL3-deficient human subjects. This lipidomic/transcriptomic data is important to consider when targeting ANGPTL3 for therapy and translating it to the human context.

\section{Acknowledgements}

We thank all the volunteers who participated in this study. We also thank Meghana Nagaraj, MSc, as well as Riikka Kosonen, MSc, and Sanna Sihvo for the technical assistance and Anna Montali, BSc, for her assistance in recruiting study subjects.

\section{Funding}

Novo Nordisk Foundation (V.M. Olkkonen), the Paavo Nurmi Foundation (V.M. Olkkonen), the Liv och Hälsa Foundation (V.M. Olkkonen), the Finnish Foundation for Cardiovascular Research (M. Jauhiainen, V.M. Olkkonen), the Jane and Aatos Erkko Foundation (M. Jauhiainen), the Magnus Ehrnrooth Foundation (M. Jauhiainen), and Progetto Ateneo 2006 and Progetto Ateneo 2011 from Sapienza University of Rome (M. Arca) are 
acknowledged for financial support. J.D is funded by European Research Council (ERC) under the European Union's Horizon 2020 research and innovation programme (grant no: 677542) and the Barts Charity (grant no: MGU0343) to J.D. J.D. is also supported by a Sir Henry Dale Fellowship jointly funded by the Wellcome Trust and the Royal Society (grant 107613/Z/15/Z)

\section{Conflicts of interest}

None.

\section{REFERENCES}

[1] WHO, 2018. World Health Organization Fact Sheet: The Top 10 Causes of Death 2019. https://www.who.int/news-room/fact-sheets/detail/the-top-10-causes-of-death. 24 May 2018 (accessed 2 January 2020).

[2] Stone, N.J., Robinson, J.G., Lichtenstein, A.H., Bairey Merz, C.N., Blum, C.B., Eckel, R.H., et al., 2014. 2013 ACC/AHA guideline on the treatment of blood cholesterol to reduce atherosclerotic cardiovascular risk in adults: a report of the American College of Cardiology/American Heart Association Task Force on Practice Guidelines. Circulation 129:S1-45.

[3] Hegele, R.A., Tsimikas, S., 2019. Lipid-Lowering Agents. Circulation research 124:386-404.

[4] Köster, A., Chao, Y.B., Mosior, M., Ford, A., Gonzalez-DeWhitt, P.A., Hale, J.E., et al., 2005. Transgenic angiopoietin-like (angptl)4 overexpression and targeted disruption of angpt/4 and angpt|3: regulation of triglyceride metabolism. Endocrinology 146:4943-4950.

[5] Gusarova, V., Banfi, S., Alexa-Braun, C.A., Shihanian, L.M., Mintah, I.J., Lee, J.S., et al., 2017. ANGPTL8 Blockade With a Monoclonal Antibody Promotes Triglyceride Clearance, Energy Expenditure, and Weight Loss in Mice. Endocrinology 158:1252-1259. 
[6] Musunuru, K., Pirruccello, J.P., Do, R., Peloso, G.M., Guiducci, C., Sougnez, C., et al., 2010. Exome sequencing, ANGPTL3 mutations, and familial combined hypolipidemia. The New England Journal of Medicine 363:2220-2227.

[7] Stitziel, N.O., Khera, A.V., Wang, X., Bierhals, A.J., Vourakis, A.C., Sperry, A.E., et al., 2017. ANGPTL3 Deficiency and Protection Against Coronary Artery Disease. Journal of the American College of Cardiology 69:2054-2063.

[8] Minicocci, I., Santini, S., Cantisani, V., Stitziel, N., Kathiresan, S., Arroyo, J.A., et al., 2013. Clinical characteristics and plasma lipids in subjects with familial combined hypolipidemia: a pooled analysis. Journal of Lipid Research 54:3481-3490.

[9] Robciuc, M.R., Maranghi, M., Lahikainen, A., Rader, D., Bensadoun, A., Oorni, K., et al., 2013. Angptl3 deficiency is associated with increased insulin sensitivity, lipoprotein lipase activity, and decreased serum free fatty acids. Arteriosclerosis, Thrombosis, and Vascular Biology 33:17061713.

[10] Lawson, T., 2011. A Year in the Village of Eternity: the Lifestyle of Longevity in Campodimele, Italy. 1st ed. New York: Bloomsbury.

[11] Minicocci, I., Montali, A., Robciuc, M.R., Quagliarini, F., Censi, V., Labbadia, G., et al., 2012. Mutations in the ANGPTL3 gene and familial combined hypolipidemia: a clinical and biochemical characterization. The Journal of Clinical Endocrinology and Metabolism 97:E1266-75.

[12] Dewey, F.E., Gusarova, V., Dunbar, R.L., O'Dushlaine, C., Schurmann, C., Gottesman, O., et al., 2017. Genetic and Pharmacologic Inactivation of ANGPTL3 and Cardiovascular Disease. The New England Journal of Medicine 377:211-221.

[13] Gusarova, V., Alexa, C.A., Wang, Y., Rafique, A., Kim, J.H., Buckler, D., et al., 2015. ANGPTL3 blockade with a human monoclonal antibody reduces plasma lipids in dyslipidemic mice and monkeys. Journal of Lipid Research 56:1308-1317. 
[14] Graham, M.J., Lee, R.G., Brandt, T.A., Tai, L.J., Fu, W., Peralta, R., et al., 2017. Cardiovascular and Metabolic Effects of ANGPTL3 Antisense Oligonucleotides. The New England Journal of Medicine 377:222-232.

[15] Romeo, S., Yin, W., Kozlitina, J., Pennacchio, L.A., Boerwinkle, E., Hobbs, H.H., et al., 2009. Rare loss-of-function mutations in ANGPTL family members contribute to plasma triglyceride levels in humans. The Journal of Clinical Investigation 119:70-79.

[16] Nidhina Haridas, P.A., Soronen, J., Sädevirta, S., Mysore, R., Quagliarini, F., Pasternack, A., et al., 2015. Regulation of Angiopoietin-Like Proteins (ANGPTLs) 3 and 8 by Insulin. The Journal of Clinical Endocrinology and Metabolism 100:E1299-307.

[17] Shimizugawa, T., Ono, M., Shimamura, M., Yoshida, K., Ando, Y., Koishi, R., et al., 2002. ANGPTL3 decreases very low density lipoprotein triglyceride clearance by inhibition of lipoprotein lipase. The Journal of Biological Chemistry 277:33742-33748.

[18] Shimamura, M., Matsuda, M., Yasumo, H., Okazaki, M., Fujimoto, K., Kono, K., et al., 2007. Angiopoietin-like protein3 regulates plasma HDL cholesterol through suppression of endothelial lipase. Arteriosclerosis, Thrombosis, and Vascular Biology 27:366-372.

[19] Wang, Y., Gusarova, V., Banfi, S., Gromada, J., Cohen, J.C., Hobbs, H.H., 2015. Inactivation of ANGPTL3 reduces hepatic VLDL-triglyceride secretion. Journal of Lipid Research 56:12961307.

[20] Xu, Y.X., Redon, V., Yu, H., Querbes, W., Pirruccello, J., Liebow, A., et al., 2018. Role of angiopoietin-like 3 (ANGPTL3) in regulating plasma level of low-density lipoprotein cholesterol. Atherosclerosis 268:196-206.

[21] Shimamura, M., Matsuda, M., Kobayashi, S., Ando, Y., Ono, M., Koishi, R., et al., 2003. Angiopoietin-like protein 3, a hepatic secretory factor, activates lipolysis in adipocytes. Biochemical and Biophysical Research Communications 301:604-609. 
[22] Tikkanen, E., Minicocci, I., Hällfors, J., Di Costanzo, A., D'Erasmo, L., Poggiogalle, E., et al., 2019. Metabolomic Signature of Angiopoietin-Like Protein 3 Deficiency in Fasting and Postprandial State. Arteriosclerosis, Thrombosis, and Vascular Biology 39:665-674.

[23] Tikka, A., Soronen, J., Laurila, P.P., Metso, J., Ehnholm, C., Jauhiainen, M., 2014. Silencing of ANGPTL 3 (angiopoietin-like protein 3) in human hepatocytes results in decreased expression of gluconeogenic genes and reduced triacylglycerol-rich VLDL secretion upon insulin stimulation. Bioscience Reports 34:e00160.

[24] Yu, G., Wang, L.G., Han, Y., He, Q.Y., 2012. clusterProfiler: an R package for comparing biological themes among gene clusters. Omics : a journal of integrative biology 16:284-287.

[25] Csárdi, G., Nepusz, T., 2006. The igraph software package for complex network research. InterJournal Complex Systems 1695:1-9.

[26] Shannon, P., Markiel, A., Ozier, O., Baliga, N.S., Wang, J.T., Ramage, D., et al., 2003. Cytoscape: a software environment for integrated models of biomolecular interaction networks. Genome Research 13:2498-2504.

[27] Minicocci, I., Tikka, A., Poggiogalle, E., Metso, J., Montali, A., Ceci, F., et al., 2016. Effects of angiopoietin-like protein 3 deficiency on postprandial lipid and lipoprotein metabolism. Journal of Lipid Research 57:1097-1107.

[28] Hilvo, M., Simolin, H., Metso, J., Ruuth, M., Öörni, K., Jauhiainen, M., et al., 2018. PCSK9 inhibition alters the lipidome of plasma and lipoprotein fractions. Atherosclerosis 269:159-165.

[29] Folch, J., Lees, M., Sloane Stanley, G.H., 1957. A simple method for the isolation and purification of total lipides from animal tissues. The Journal of Biological Chemistry 226:497-509.

[30] Ruhanen, H., Nidhina Haridas, P.A., Eskelinen, E.L., Eriksson, O., Olkkonen, V.M., Käkelä, R., 2017. Depletion of TM6SF2 disturbs membrane lipid composition and dynamics in HuH7 hepatoma cells. Biochimica et Biophysica Acta - Molecular and Cell Biology of Lipids 1862:676-685.

[31] Haimi, P., Uphoff, A., Hermansson, M., Somerharju, P., 2006. Software tools for analysis of mass spectrometric lipidome data. Analytical Chemistry 78:8324-8331. 
[32] Käkelä, R., Käkelä, A., Kahle, S., Becker, B.H., Kelly, A., Furness, R.W., 2005. Fatty acid signatures in plasma of captive herring gulls as indicators of demersal or pelagic fish diet. Marine Ecology Progress Series. 293:191-200.

[33] Bligh, E.G., Dyer, W.J., 1959. A rapid method of total lipid extraction and purification. Canadian Journal of Biochemistry and Physiology 37:911.

[34] Dalli, J., Colas, R.A., Walker, M.E., Serhan, C.N., 2018. Lipid Mediator Metabolomics Via LCMS/MS Profiling and Analysis. Methods in molecular biology (Clifton, N.J.) 1730:59-72.

[35] Wold, S., Sjöström, M., 1977. SIMCA: A Method for Analyzing Chemical Data in Terms of Similarity and Analogy. In: Kowalski, B., editor. Chemometrics: Theory and Application, Washington D.C.: American Chemical Society, p. 243-282.

[36] Cugini, P., Leone, G., Lucia, P., Sepe, F.A., Pelosio, A., Caparelli, T., et al., 1994. Campodimele study: blood pressure and heart rate pattern in clinically healthy elderly subjects. Chronobiology International 11:381-392.

[37] Ichi, I., Kono, N., Arita, Y., Haga, S., Arisawa, K., Yamano, M., et al., 2014. Identification of genes and pathways involved in the synthesis of Mead acid (20:3n-9), an indicator of essential fatty acid deficiency. Biochimica et Biophysica Acta - Molecular and Cell Biology of Lipids $1841: 204-213$.

[38] Kane, C.D., Coe, N.R., Vanlandingham, B., Krieg, P., Bernlohr, D.A., 1996. Expression, purification, and ligand-binding analysis of recombinant keratinocyte lipid-binding protein (MAL-1), an intracellular lipid-binding found overexpressed in neoplastic skin cells. Biochemistry 35:28942900.

[39] Murphy, E.J., Owada, Y., Kitanaka, N., Kondo, H., Glatz, J.F., 2005. Brain arachidonic acid incorporation is decreased in heart fatty acid binding protein gene-ablated mice. Biochemistry 44:6350-6360. 
[40] Ehehalt, R., Sparla, R., Kulaksiz, H., Herrmann, T., Fullekrug, J., Stremmel, W., 2008. Uptake of long chain fatty acids is regulated by dynamic interaction of FAT/CD36 with cholesterol/sphingolipid enriched microdomains (lipid rafts). BMC Cell Biology 9:45-2121-9-45.

[41] Islam, A., Kagawa, Y., Sharifi, K., Ebrahimi, M., Miyazaki, H., Yasumoto, Y., et al., 2014. Fatty Acid Binding Protein 3 Is Involved in $n-3$ and $n-6$ PUFA transport in mouse trophoblasts. The Journal of Nutrition 144:1509-1516.

[42] Shikano, M., Masuzawa, Y., Yazawa, K., Takayama, K., Kudo, I., Inoue, K., 1994. Complete discrimination of docosahexaenoate from arachidonate by $85 \mathrm{kDa}$ cytosolic phospholipase A2 during the hydrolysis of diacyl- and alkenylacylglycerophosphoethanolamine. Biochimica et Biophysica Acta - Lipids and Lipid Metabolism 1212:211-216.

[43] Batchu, K.C., Hänninen, S., Jha, S.K., Jeltsch, M., Somerharju, P., 2016. Factors regulating the substrate specificity of cytosolic phospholipase A2-alpha in vitro. Biochimica et Biophysica Acta - Molecular and Cell Biology of Lipids 1861:1597-1604.

[44] Norris, P.C., Gosselin, D., Reichart, D., Glass, C.K., Dennis, E.A., 2014. Phospholipase A2 regulates eicosanoid class switching during inflammasome activation. Proceedings of the National Academy of Sciences of the United States of America 111:12746-12751.

[45] Hicks, A.M., DeLong, C.J., Thomas, M.J., Samuel, M., Cui, Z., 2006. Unique molecular signatures of glycerophospholipid species in different rat tissues analyzed by tandem mass spectrometry. Biochimica et Biophysica Acta - Molecular and Cell Biology of Lipids 1761:10221029.

[46] D'Souza, K., Epand, R.M., 2014. Enrichment of phosphatidylinositols with specific acyl chains. Biochimica et Biophysica Acta - Biomembranes 1838:1501-1508.

[47] Traynor-Kaplan, A., Kruse, M., Dickson, E.J., Dai, G., Vivas, O., Yu, H., et al., 2017. Fatty-acyl chain profiles of cellular phosphoinositides. Biochimica et Biophysica Acta - Molecular and Cell Biology of Lipids 1862:513-522. 
[48] Elajami, T.K., Colas, R.A., Dalli, J., Chiang, N., Serhan, C.N., Welty, F.K., 2016. Specialized proresolving lipid mediators in patients with coronary artery disease and their potential for clot remodeling. FASEB journal: official publication of the Federation of American Societies for Experimental Biology 30:2792-2801.

[49] Deng, B., Wang, C.W., Arnardottir, H.H., Li, Y., Cheng, C.Y., Dalli, J., et al., 2014. Maresin biosynthesis and identification of maresin 2, a new anti-inflammatory and pro-resolving mediator from human macrophages. PloS one 9:e102362.

[50] Colas, R.A., Dalli, J., Chiang, N., Vlasakov, I., Sanger, J.M., Riley, I.R., et al., 2016. Identification and Actions of the Maresin 1 Metabolome in Infectious Inflammation. Journal of Immunology (Baltimore, Md.: 1950) 197:4444-4452.

[51] Rius, B., Duran-Guell, M., Flores-Costa, R., Lopez-Vicario, C., Lopategi, A., Alcaraz-Quiles, J., et al., 2017. The specialized proresolving lipid mediator maresin 1 protects hepatocytes from lipotoxic and hypoxia-induced endoplasmic reticulum stress. FASEB journal: official publication of the Federation of American Societies for Experimental Biology 31:5384-5398.

[52] Jung, T.W., Kyung, E.J., Kim, H.C., Shin, Y.K., Lee, S.H., Park, E.S., et al., 2018. Protectin DX Ameliorates Hepatic Steatosis by Suppression of Endoplasmic Reticulum Stress via AMPKInduced ORP150 Expression. The Journal of Pharmacology and Experimental Therapeutics 365:485-493.

[53] Jung, T.W., Ahn, S.H., Shin, J.W., Kim, H.C., Park, E.S., Abd El-Aty, A.M., et al., 2019. Protectin DX ameliorates palmitate-induced hepatic insulin resistance through AMPK/SIRT1mediated modulation of fetuin-A and SeP expression. Clinical and Experimental Pharmacology \& Physiology 46:898-909.

[54] Dakin, S.G., Colas, R.A., Newton, J., Gwilym, S., Jones, N., Reid, H.A.B., et al., 2019. 15-EpiLXA4 and MaR1 counter inflammation in stromal cells from patients with Achilles tendinopathy and rupture. FASEB journal: official publication of the Federation of American Societies for Experimental Biology 33:8043-8054. 
[55] Kain, V., Liu, F., Kozlovskaya, V., Ingle, K.A., Bolisetty, S., Agarwal, A., et al., 2017. Resolution Agonist 15-epi-Lipoxin A4 Programs Early Activation of Resolving Phase in PostMyocardial Infarction Healing. Scientific Reports 7:9999-017-10441-8.

[56] Ricciotti, E., FitzGerald, G.A., 2011. Prostaglandins and inflammation. Arteriosclerosis, Thrombosis, and Vascular Biology 31:986-1000.

[57] Levy, B.D., Clish, C.B., Schmidt, B., Gronert, K., Serhan, C.N., 2001. Lipid mediator class switching during acute inflammation: signals in resolution. Nature Immunology 2:612-619.

[58] Holopainen, M., Colas, R.A., Valkonen, S., Tigistu-Sahle, F., Hyvärinen, K., Mazzacuva, F., et al., 2019. Polyunsaturated fatty acids modify the extracellular vesicle membranes and increase the production of proresolving lipid mediators of human mesenchymal stromal cells. Biochimica et Biophysica Acta - Molecular and Cell Biology of Lipids 1864:1350-1362.

[59] Liang, J.J., Oelkers, P., Guo, C., Chu, P.C., Dixon, J.L., Ginsberg, H.N., et al., 2004. Overexpression of human diacylglycerol acyltransferase 1, acyl-coa:cholesterol acyltransferase 1, or acyl-CoA:cholesterol acyltransferase 2 stimulates secretion of apolipoprotein B-containing lipoproteins in McA-RH7777 cells. The Journal of Biological Chemistry 279:44938-44944.

[60] Burnett, J.R., Wilcox, L.J., Telford, D.E., Kleinstiver, S.J., Barrett, P.H., Newton, R.S., et al., 1999. Inhibition of ACAT by avasimibe decreases both VLDL and LDL apolipoprotein B production in miniature pigs. Journal of Lipid Research 40:1317-1327.

[61] Terasaka, N., Miyazaki, A., Kasanuki, N., Ito, K., Ubukata, N., Koieyama, T., et al., 2007. ACAT inhibitor pactimibe sulfate (CS-505) reduces and stabilizes atherosclerotic lesions by cholesterol-lowering and direct effects in apolipoprotein E-deficient mice. Atherosclerosis 190:239247.

[62] Wang, C.S., Bass, H., Whitmer, R., McConathy, W.J., 1993. Effects of albumin and apolipoprotein C-II on the acyl-chain specificity of lipoprotein lipase catalysis. Journal of Lipid Research 34:2091-2098. 
[63] Sato, K., Takahashi, T., Takahashi, Y., Shiono, H., Katoh, N., Akiba, Y., 1999. Preparation of chylomicrons and VLDL with monoacid-rich triacylglycerol and characterization of kinetic parameters in lipoprotein lipase-mediated hydrolysis in chickens. The Journal of Nutrition 129:126131.

[64] McLean, L.R., Demel, R.A., Socorro, L., Shinomiya, M., Jackson, R.L., 1986. Mechanism of action of lipoprotein lipase. Methods in Enzymology 129:738-763.

[65] Olivecrona, T., Bengtsson-Olivecrona, G., 1987. Lipoprotein lipase from milk - The model enzyme in lipoprotein lipase research. In: Borensztajn, J., editor. Lipoprotein lipase, Chicago: Evener Publishers, p. 15-58.

[66] Dugi, K.A., Dichek, H.L., Talley, G.D., Brewer, H.B.,Jr, Santamarina-Fojo, S., 1992. Human lipoprotein lipase: the loop covering the catalytic site is essential for interaction with lipid substrates. The Journal of Biological Chemistry 267:25086-25091.

[67] Glomset, J.A., 1979. Lecithin: cholesterol acyltransferase. An exercise in comparative biology. Progress in Biochemical Pharmacology 15:41-66.

[68] Liu, M., Bagdade, J.D., Subbaiah, P.V., 1995. Specificity of lecithin:cholesterol acyltransferase and atherogenic risk: comparative studies on the plasma composition and in vitro synthesis of cholesteryl esters in 14 vertebrate species. Journal of Lipid Research 36:1813-1824.

[69] Subbaiah, P.V., Sowa, J.M., Davidson, M.H., 2004. Evidence for altered positional specificity of LCAT in vivo: studies with docosahexaenoic acid feeding in humans. Journal of Lipid Research $45: 2245-2251$.

[70] McLean, L.R., Best, S., Balasubramaniam, A., Jackson, R.L., 1986. Fatty acyl chain specificity of phosphatidylcholine hydrolysis catalyzed by lipoprotein lipase. Effect of apolipoprotein C-II and its (56-79) synthetic fragment. Biochimica et Biophysica Acta - Lipids and Lipid Metabolism 878:446-449.

[71] van den Bosch, H., Schrakamp, G., Hardeman, D., Zomer, A.W., Wanders, R.J., Schutgens, R.B., 1993. Ether lipid synthesis and its deficiency in peroxisomal disorders. Biochimie 75:183-189. 
[72] Griffon, N., Budreck, E.C., Long, C.J., Broedl, U.C., Marchadier, D.H., Glick, J.M., et al., 2006. Substrate specificity of lipoprotein lipase and endothelial lipase: studies of lid chimeras. Journal of Lipid Research 47:1803-1811.

[73] Huuskonen, J., Olkkonen, V.M., Jauhiainen, M., Metso, J., Somerharju, P., Ehnholm, C., 1996. Acyl chain and headgroup specificity of human plasma phospholipid transfer protein. Biochimica et Biophysica Acta - Lipids and Lipid Metabolism 1303:207-214.

[74] Ruuth, M., Nguyen, S.D., Vihervaara, T., Hilvo, M., Laajala, T.D., Kondadi, P.K., et al., 2018. Susceptibility of low-density lipoprotein particles to aggregate depends on particle lipidome, is modifiable, and associates with future cardiovascular deaths. European Heart Journal 39:25622573.

[75] Horter, M.J., Sondermann, S., Reinecke, H., Bogdanski, J., Woltering, A., Kerber, S., et al., 2002. Associations of HDL phospholipids and paraoxonase activity with coronary heart disease in postmenopausal women. Acta Physiologica Scandinavica 176:123-130.

[76] Subbaiah, P.V., Liu, M., 1993. Role of sphingomyelin in the regulation of cholesterol esterification in the plasma lipoproteins. Inhibition of lecithin-cholesterol acyltransferase reaction. The Journal of Biological Chemistry 268:20156-20163.

[77] Parini, P., Davis, M., Lada, A.T., Erickson, S.K., Wright, T.L., Gustafsson, U., et al., 2004. ACAT2 is localized to hepatocytes and is the major cholesterol-esterifying enzyme in human liver. Circulation 110:2017-2023.

[78] Akagi, D., Chen, M., Toy, R., Chatterjee, A., Conte, M.S., 2015. Systemic delivery of proresolving lipid mediators resolvin D2 and maresin 1 attenuates intimal hyperplasia in mice. FASEB journal: official publication of the Federation of American Societies for Experimental Biology 29:2504-2513.

[79] Fredman, G., Hellmann, J., Proto, J.D., Kuriakose, G., Colas, R.A., Dorweiler, B., et al., 2016. An imbalance between specialized pro-resolving lipid mediators and pro-inflammatory leukotrienes promotes instability of atherosclerotic plaques. Nature Communications 7:12859. 
[80] Gerlach, B.D., Marinello, M., Heinz, J., Rymut, N., Sansbury, B.E., Riley, C.O., et al., 2020. Resolvin D1 promotes the targeting and clearance of necroptotic cells. Cell Death \& differentiation 27:525-539. 
Table 1. Several KEGG pathways related to lipid metabolism are altered in ANGPLT3 knock-down hepatocytes. A total of 171 pathways were obtained in the KEGG pathway enrichment analysis, and a full list of pathways and statistical values is available as Supplementary table 3.

\begin{tabular}{llll}
$\begin{array}{l}\text { Ranking } \\
(/ \mathbf{1 7 1})\end{array}$ & Pathway & p value & $\begin{array}{l}\text { Adjusted } \\
\text { p value }\end{array}$ \\
\hline $\mathbf{1}$ & Inositol phosphate metabolism & $2.329 \mathrm{E}-25$ & $7.196 \mathrm{E}-23$ \\
$\mathbf{2}$ & Phosphatidylinositol signaling system & $1.581 \mathrm{E}-23$ & $2.442 \mathrm{E}-21$ \\
$\mathbf{3}$ & Fatty acid metabolism & $1.198 \mathrm{E}-22$ & $1.234 \mathrm{E}-20$ \\
$\mathbf{4}$ & Glycerophospholipid metabolism & $1.172 \mathrm{E}-19$ & $9.055 \mathrm{E}-18$ \\
$\mathbf{6}$ & AMPK signaling pathway & $3.653 \mathrm{E}-18$ & $1.881 \mathrm{E}-16$ \\
$\mathbf{7}$ & Biosynthesis of unsaturated fatty acids & $1.133 \mathrm{E}-17$ & $5.002 \mathrm{E}-16$ \\
$\mathbf{1 0}$ & Phospholipase D signaling pathway & $2.606 \mathrm{E}-16$ & $8.053 \mathrm{E}-15$ \\
$\mathbf{1 3}$ & PPAR signaling pathway & $6.831 \mathrm{E}-16$ & $1.624 \mathrm{E}-14$ \\
$\mathbf{1 4}$ & Sphingolipid signaling pathway & $1.780 \mathrm{E}-15$ & $3.929 \mathrm{E}-14$ \\
$\mathbf{1 7}$ & Sphingolipid metabolism & $1.107 \mathrm{E}-14$ & $2.012 \mathrm{E}-13$ \\
$\mathbf{1 9}$ & Fatty acid elongation & $2.556 \mathrm{E}-13$ & $4.157 \mathrm{E}-12$ \\
$\mathbf{2 9}$ & Peroxisome & $1.579 \mathrm{E}-10$ & $1.683 \mathrm{E}-09$ \\
$\mathbf{3 1}$ & Cholesterol metabolism & $4.352 \mathrm{E}-10$ & $4.338 \mathrm{E}-09$ \\
$\mathbf{4 4}$ & Ether lipid metabolism & $2.027 \mathrm{E}-08$ & $1.423 \mathrm{E}-07$ \\
$\mathbf{4 5}$ & Fatty acid degradation & $2.497 \mathrm{E}-08$ & $1.715 \mathrm{E}-07$ \\
$\mathbf{4 8}$ & Glycerolipid metabolism & $8.699 \mathrm{E}-08$ & $5.600 \mathrm{E}-07$ \\
$\mathbf{9 0}$ & Steroid biosynthesis & $1.397 \mathrm{E}-05$ & $4.795 \mathrm{E}-05$ \\
$\mathbf{1 1 2}$ & ABC transporters & $3.483 \mathrm{E}-04$ & $9.608 \mathrm{E}-04$ \\
$\mathbf{1 1 3}$ & Fatty acid biosynthesis & $3.866 \mathrm{E}-04$ & $1.057 \mathrm{E}-03$ \\
$\mathbf{1 2 1}$ & Arachidonic acid metabolism & $1.075 \mathrm{E}-03$ & $2.744 \mathrm{E}-03$
\end{tabular}




\section{Figure legends}

Figure 1. ANGPTL3 knock-down efficiency in immortalized human hepatocytes. (A) ANGPTL3 mRNA expression of $\mathrm{IHH}$ cells treated with non-targeting shRNA (control) and shRNA targeting ANGPTL3 (shANGPTL3). The values represent mean $\pm S D, n=3$. (B) ANGPTL3 protein levels in control and ANGPTL3 knock-down cells (data represent mean fold change $\pm S D, n=3$ ). (C) Representative western blot image of ANGPTL3 expression in control and knock-down cells with corresponding total protein. ${ }^{* *} p<0.001,{ }^{*} p<0.05$.

Figure 2. MUFAs are decreased and PUFAs increased in ANGPTL3 knock-down cells. (A) Sums of saturated and unsaturated fatty acids determined by gas chromatography. The values represent mean $\pm S D, n=6 .{ }^{* *} p<0.01,{ }^{* * *} p<0.001$. (B) PCA of individual FA species. The samples located furthest from the origin of the PCA biplot (marked as + ) on one side contain relatively more of the lipid species furthest on that same side. The longer the distance between two samples on the plot the more their lipid profiles differ from each other. The percentages represent the proportion of the variation in the data each principal component axis explains. Ctrl=control cells treated with nontargeting RNA, shANGPTL3/ShA3=cells treated with shRNA against ANGTPL3.

Figure 3. Knocking down ANGPTL3 enhances the production of lipid mediators. (A) PLS-DA analysis of lipid mediator concentrations. The score plot shows clear separation between the control (red dots) and knock-down (shANGPTL3; green dots) samples. Coloured spherical areas display the $95 \%$ confidence region. (B) VIP scores of lipid mediator variables that contribute most to the separation in the PLS-DA model. All of these mediators are more abundant in the ANGPTL3 knock-down (shA3) cells as indicated by the colour scale. RvD6=resolvin D6, $\mathrm{PGD}_{2}=$ prostaglandin $D_{2}, T_{x} B_{2}=$ thromboxane $B_{2}, H D P A=$ hydroxydocosapentaenoic acid, MaR=maresin, $L X A_{4}=$ lipoxin $A_{4}$, $\mathrm{LTB}_{4}=$ leukotriene $\mathrm{B}_{4}, \mathrm{HDHA}=$ hydroxydocosahexaenoic acid, HETE=hydroxyeicosatetraenoic acid.

Figure 4. Cholesterol ester synthesis and SOAT1 expression are reduced in ANGPTL3 cells. (A) Total cholesterol esters (CE) in control and ANGPTL3 knock-down cells (data represent mean \pm SD, $n=6$ ). (B) SOAT1 mRNA expression in control and ANGPTL3 knock-down cells (mean \pm SD, 
$\mathrm{n}=6$ ) (C) representative Western blot of SOAT1 in control and ANGPTL3 knock-down cells (D) Quantification of protein expression of SOAT1 in ANGPTL3 knock-down and control cells (mean \pm $\mathrm{SD}, \mathrm{n}=4)$. (E) $\left[{ }^{3} \mathrm{H}\right]$ acetic acid labeled CE in control and ANGPTL3 knock-down cells (mean $\pm \mathrm{SD}$, $\mathrm{n}=12$ ). (F) $\left[{ }^{3} \mathrm{H}\right.$ ]oleic acid incorporation into CE in control and ANGPTL3 knock-down cells (mean \pm $\mathrm{SD}, \mathrm{n}=18) .{ }^{* *} \mathrm{p}<0.001,{ }^{* *} \mathrm{p}<0.001$.

Figure 5. ANGPTL3 LOF carriers have altered lipoprotein fatty acid profiles. PCA of the lipoprotein fatty acid profiles of control and ANGPTL3 LOF subjects. Lipoprotein fractions were isolated from the plasma of ANGPTL3 LOF homozygote and control subjects by sequential density ultracentrifugation and fatty acids were analyzed by gas chromatography. Sample markings: Group+Fraction; $\mathrm{C}=$ control, $\mathrm{HZ}=\mathrm{ANGPTL3}$ LOF homozygote, $\mathrm{V}=\mathrm{VLDL}, \quad \mathrm{L}=\mathrm{LDL}, \quad \mathrm{H}=\mathrm{HDL}$. $\mathrm{DMA}=$ dimethylacetal.

Figure 6. PUFAs accumulate in TAGs of lipoproteins derived from ANGPTL3 LOF homozygotes. TAG of lipoproteins from ANGPTL3 loss-of-function and control subjects was analyzed by ESIMS/MS. Bar graphs are shown for VLDL (upper panel) and LDL (lower panel) fractions, but also in HDL the effect was clear and very similar to that seen in LDL (Supplementary table 12). The values represent mean $\pm S D, n=4-10 .{ }^{*} p<0.05,{ }^{* *} p<0.01,{ }^{* * *} p<0.001$.

Figure 7. ANGPTL3 deficiency alters the surface lipids of lipoprotein particles. (A) SM/PC ratio of different lipoprotein fractions. ANGPTL3=ANGPTL3 LOF homozygote. (B) PCA of the relative profile of SM in LDL. C=control, HZ=ANGPTL3 LOF homozygote. All species have a sphingosine 18:1 backbone, except SM 24:2 is likely a mixture of 18:1/24:2 and 18:2/24:1. (C) LysoPC species profile of LDL derived from control subjects and ANGPTL3 LOF homozygotes. The values represent mean $\pm S D, n=5-10 .{ }^{*} p<0.05,{ }^{* *} p<0.01,{ }^{* * *} p<0.001$. 


\section{ANGPTL3 deficiency alters the lipid profile and metabolism of cultured hepatocytes and human lipoproteins}

Hanna Ruhanen ${ }^{1,2,3}$, Nidhina Haridas P.A. ${ }^{1}$, Ilenia Minicocci ${ }^{4}$, Juuso H. Taskinen ${ }^{1}$, Francesco Palmas ${ }^{5}$, Alessia di Costanzo ${ }^{4}$, Laura D’Erasmo ${ }^{4}$, Jari Metso ${ }^{1}$, Jennimari Partanen ${ }^{1}$, Jesmond Dalli, ${ }^{5,6}$, You Zhou ${ }^{7}$, Marcello Arca ${ }^{4}$, Matti Jauhiainen ${ }^{1}$, Reijo Käkelä ${ }^{2,3}$ \& Vesa M. Olkkonen ${ }^{1,8, *}$

${ }^{1}$ Minerva Foundation Institute for Medical Research, Helsinki, Finland; ${ }^{2}$ Molecular and Integrative Biosciences, University of Helsinki, Helsinki, Finland; ${ }^{3}$ Helsinki University Lipidomics Unit (HiLIPID), Helsinki Institute for Life Science (HiLIFE), Helsinki, Finland; ${ }^{4}$ Department of Translational and Precision Medicine, Sapienza University of Rome, Italy, ${ }^{5}$ Lipid Mediator Unit, William Harvey Research Institute, Barts and the London School of Medicine and Dentistry, Queen Mary University of London, London, United Kingdom; ${ }^{6}$ Centre for Inflammation and Therapeutic Innovation, Queen Mary University of London, London, UK;

${ }^{7}$ Systems Immunity University Research Institute and Division of Infection \& Immunity, Cardiff University, Cardiff, United Kingdom, ${ }^{8}$ Department of Anatomy, University of Helsinki, Finland.

${ }^{*}$ Corresponding author at: Minerva Foundation Institute for Medical Research, Biomedicum 2U, Tukholmankatu 8, FI-00290 Helsinki, Finland; Tel +358-2-94125705, e-mail vesa.olkkonen@helsinki.fi 


\section{ABSTRACT}

Loss-of-function (LOF) mutations in ANGPTL3, an inhibitor of lipoprotein lipase (LPL), cause a drastic reduction of serum lipoproteins and protect against the development of atherosclerotic cardiovascular disease. Therefore, ANGPTL3 is a promising therapy target. We characterized the impacts of ANGPTL3 depletion on the immortalized human hepatocyte $(\mathrm{IHH})$ transcriptome, lipidome and human plasma lipoprotein lipidome. The transcriptome of ANGPTL3 knock-down (KD) cells showed altered expression of several pathways related to lipid metabolism. Accordingly, ANGPTL3 depleted IHH displayed changes in cellular overall fatty acid (FA) composition and in the lipid species composition of several lipid classes, characterized by abundant $n-6$ and $n-3$ polyunsaturated FAs (PUFAs). This PUFA increase coincided with an elevation of lipid mediators, among which there were species relevant for resolution of inflammation, protection from lipotoxic and hypoxia-induced ER stress, hepatic steatosis and insulin resistance or for the recovery from cardiovascular events. Cholesterol esters were markedly reduced in ANGPTL3 KD $\mathrm{IHH}$, coinciding with suppression of the SOAT1 mRNA and protein. ANGPTL3 LOF caused alterations in plasma lipoprotein FA and lipid species composition. All lipoprotein fractions of the ANGPTL3 LOF subjects displayed a marked drop of 18:2n-6, while several highly unsaturated triacylglycerol (TAG) species were enriched. The present work reveals distinct impacts of ANGPTL3 depletion on the hepatocellular lipidome, transcriptome and lipid mediators, as well as on the lipidome of lipoproteins isolated from plasma of ANGPTL3deficient human subjects. It is important to consider these lipidomics and transcriptomics findings when targeting ANGPTL3 for therapy and translating it to the human context.

Keywords: Angiopoietin like 3, lipoprotein lipase, lipidomics, lipid metabolism, cholesterol ester, polyunsaturated fatty acid 


\section{Introduction}

Cardiovascular diseases (CVD) are one of the leading causes of death worldwide [1]. Dyslipidemia, mainly elevated low-density-lipoprotein (LDL) cholesterol and triglyceride rich lipoproteins (TRLs), is the major risk factor for the development of CVD. Statins and ezetimibe, which reduce LDL-cholesterol, are used as the most common standard therapeutics for CVD treatment [2]. However due to a high level of residual CVD risk refractory to these therapies, efforts have been made to identify novel targets and treatment modalities for CVD [3]. Angiopoietin like protein 3 (ANGPTL3) is one of the main targets in this line of research. Among the ANGPTL family of proteins ANGPTL3, 4 and 8, play crucial roles in lipoprotein metabolism. They are potent inhibitors of LPL, which hydrolyzes triglyceride rich lipoproteins (TRLs) [4;5]. Loss of function (LOF) mutations of the ANGPTL3 gene were initially found in human subjects with extremely low serum triglycerides [6] and were later found to confer protection from coronary artery disease [7]. The ANGPTL3 LOF variant carriers exhibit hypolipidemia and high insulin sensitivity [8;9]. They have low levels of all major plasma lipoprotein classes (VLDL, LDL and HDL) [8;9]. Accordingly, this disorder was designated as a familial combined hypolipidemia (FHBL2; OMIM \#605019) [6;8]. Moreover, plasma free fatty acid (free FA) level is reduced in the ANGPTL3 LOF carriers [9]. ANGPTL3 LOF variant carriers are not known to exhibit any adverse health effects and they seem to display an increased life expectancy [10;11]. ANGPTL3 deficient subjects have been reported to lack significant coronary atherosclerotic plaques and also heterozygous ANGPTL3 LOF variant carriers display 35$40 \%$ attenuated risk of CAD compared with the general population [7;12]. ANGPTL3 has therefore emerged as a potential therapeutic target for cardiovascular disorders. A human monoclonal antibody against ANGPTL3, REGN1500, was shown to lower plasma lipids in monkeys [13]. In addition, human subjects as well as mice treated with the anti-ANGPTL3 
antibody Evinacumab exhibited hypolipidemia [12]. Similar results were obtained in both humans and mice when ANGPTL3 was silenced using an antisense-oligonucleotide approach [14].

ANGPTL3 is a protein secreted by hepatocytes [15], and insulin downregulates its expression in liver and plasma [16]. Of note, ANGPTL3 acts as an inhibitor of both LPL and endothelial lipase (EL) [17;18]. Inactivation or suppression of ANGPTL3 reduces plasma lipids by enhancing LPL activity, reducing hepatic VLDL-triglyceride secretion and enhancing LDL/VLDL uptake by the liver [19;20]. The enhancement of EL activity upon ANGPTL3 inhibition may explain the reduction of HDL observed in ANGPTL3 LOF carriers [18]. ANGPTL3 was also reported to induce adipocyte lipolysis [21], which provides a putative explanation for the reduction of FAs in the plasma of the LOF carriers [9].

Even though the functions of ANGPTL3 in the circulation are relatively well characterized, many mechanistic questions regarding the molecular consequences of ANGPTL3 LOF and protection against CVD remain unanswered. Although recent metabolic signature of lipoprotein classes from ANGPTL3 deficient subjects based on NMR [22] introduced some novel observations, the detailed lipid composition of the plasma lipoproteins of these subjects and how it may be linked to CVD protection are unclear. Likewise, the intracellular role of ANGPTL3 as a regulator of the hepatic lipid composition and function have not been studied in detail. To aid in understanding the molecular mechanisms exerted by ANGPTL3 deficiency, we addressed the alterations of hepatocellular and plasma lipid molecular species profiles caused by ANGPTL3 LOF. 


\section{Material and Methods}

2.1 Cell culture and generation of immortalized human hepatocytes (IHHs) with ANGPTL3 stably knocked down

IHHs immortalized by SV40 large T-Antigen (IHH, ATCC® PTA-5565TM) were cultured in Williams E medium (Gibco by Life Technologies, 22551-022) with added 10\%(v/v) fetal bovine serum (FBS), $0.2 \mathrm{mg} / \mathrm{ml}$ glutamine, $100 \mu \mathrm{g} / \mathrm{ml}$ streptomycin and $100 \mathrm{U} / \mathrm{ml}$ penicillin. The cells were transduced with MISSION ${ }^{T M}$ shRNA (short hairpin RNA) Lentiviral Vector particles (TRCN0000242782, Sigma Aldrich) targeting ANGPTL3 (NM_014495.2), validated in Tikka et al. [23], or with a non-targeting shRNA (SHCOO2, Sigma Aldrich) [MOI (multiplicity of infection) 1]. Transduced cells were selected with $5 \mu \mathrm{g} / \mathrm{ml}$ puromycin and cultured further in the above medium containing puromycin.

\subsection{Gene expression analysis (qPCR)}

Expression of ANGPTL3 and SOAT1 genes were analyzed by quantitative polymerase chain reaction ( $q P C R$ ). Total RNA was extracted from the ANGPTL3 KD and control IHH cells using PureLink ${ }^{\circledR}$ RNA Mini Kit according to the manufacturer's protocol. cDNA synthesis was carried out using SuperScript ${ }^{\circledR}$ VILO ${ }^{T M}$ reverse transcriptase kit (Invitrogen, 11754050). Quantitative real time PCR was performed with gene-specific primers (Supplementary table 1) and LightCyclerß 480 SYBR Green I Master mix (Roche, 04707516001) using Light Cycler 480 II instrument (Roche Applied Science, Penzberg, Germany). $\beta$-actin and RPLP0 (Large Ribosomal Protein) were used as housekeeping references to which the data were normalized. 


\subsection{Western blotting}

ANGPTL3 silenced IHH hepatocytes and control cells were lysed in RIPA buffer (15 mM Tris- $\mathrm{HCl}, \mathrm{pH} 7.4,1.25 \%$ sodium deoxycholate, 1\% NP40, 1\% SDS, 1 mM EDTA, $150 \mathrm{mM}$ $\mathrm{NaCl})$. Proteins were resolved on $12 \%$ sodium dodecyl sulfate polyacrylamide gels and blotted onto PVDF membrane using BioRad transblot system. Blots were probed with antiANGPTL3 (R332), an antibody developed in New Zealand White rabbits against a peptide EDQYKQLNQQHSQIKEIENQ of human ANGPTL3, or anti-SOAT1 (Santa Cruz sc69836), in Tris buffered saline containing 5\% bovine serum albumin and $0.1 \%$ Tween 20 . Enhanced chemiluminescence reagent (BioRad Clarity Max ${ }^{\mathrm{TM}}$ or Thermo Scientific, Waltham, MA) was used to develop and BioRad Chemidoc imaging system was used to capture the signals. Protein bands were normalized to the total protein content of the corresponding lane using Image Lab software (BioRad).

\subsection{Next generation RNA sequencing}

$\mathrm{IHH}$ cells were cultured on 6 -well plates $(n=4)$ in the conditions specified above for $48 \mathrm{~h}$, after which they were changed into fresh growth medium without puromycin and incubated for $4 \mathrm{~h}$. RNA was extracted from the ANGPTL3 KD and control IHH cells using RNeasy ${ }^{\circledR}$ Mini Kit (Qiagen, 74104) according to manufacturer's protocol.

NEBNext Ultra Directional RNA Library Prep Kit for Illumina was used to generate cDNA libraries for next generation sequencing. The mRNA was purified using magnetic beads and then fragmented in order to generate inserts of approximately $200 \mathrm{bp}$, and primed with random primers. The first strand cDNA synthesis utilized Actinomycin $\mathrm{D}$, which inhibits the DNA polymerase activity of the reverse transcriptase increasing strand specificity. In the second strand cDNA synthesis dUTP labelled oligo nucleotides were incorporated to mark 
the second strand with uracils $(U)$. The cDNA synthesis product was purified with Agencourt AMPure XP beads. Next, the cDNA was end-repaired, and adapter ligated utilizing dA-tailing. The adaptor ligated DNA went through a bead-based size selection after which the final PCR enrichment took place. Each sample was given a unique index to enable pooling of multiple samples (multiplexing) for sequencing. During the high-fidelity PCR, USER (Uracil-specific Excision Reagent) enzyme cut away the uracil strand preserving only the first strand. In addition, the loop adaptor was cut open to enable the PCR. The amplified library was then purified using AMPure XP Beads. Library quality was assessed by Bioanalyzer (Agilent DNA High Sensitivity chip) and library quantity by Qubit (Invitrogen). Sequencing was performed with Illumina NextSeq system, High Output 75 cycles kit.

STAR 2.5.1b (PMID: 23104886) was used for aligning output sequencing data to human reference genome (GRCh38) and "featureCounts" program from the Subread 1.5.0 (PMID:24227677) to count uniquely mapped fragment against genomic features defined by the GENCODE annotation file (Homo_sapiens.GRCh38.89.gtf). Differential gene expression (PMID: 25516281) was analyzed with Deseq2 1.22.1 and Benjamini-Hochberg method was used to control for false discovery rate. The transcripts with no counts in all samples were removed. Ensembl version 98 was used for gene annotation.

Gene set enrichment analysis and gene set over-representation analysis were performed using the clusterProfiler R package [24]. Overrepresentation analysis was performed using all genes, which had an adjusted p-value $<0.05$ (Benjamini-Hochberg). Network graphs were produced using igraph [25] and the results were visualized using Cytoscape [26]. 


\subsection{Study participants and lipoprotein isolation from human plasma}

The clinical characteristics of the study participants are summarized in Supplementary table 2. The study protocol was approved by the Ethical Committee of Sapienza University of Rome, and informed consent was obtained from all the study participants. ANGPTL3 LOF homozygotes and controls were matched for age, sex and BMI. The participants selected for this study are a subpopulation of a larger cohort described in previous studies [22;27]; The groups have been reported to have comparable dietary intake, physical activity, smoking prevalence, and use of anti-inflammatory medications.

Lipoprotein fractions (VLDL, LDL and HDL) were isolated from plasma by sequential density ultracentrifugation as described previously [28].

\subsection{Lipidomics of IHH cells and lipoprotein fractions by ESI-MS/MS and gas chromatography (GC)}

For IHH lipidomics, ANGPTL3 KD and control IHH cells were first cultured on 6-well $(n=6)$ plates for $48 \mathrm{~h}$ as mentioned above. Fresh medium without puromycin was changed into the wells and cells were incubated for $24 \mathrm{~h}$, washed with ice-cold PBS and finally scraped into $1 \mathrm{ml}$ of ice-cold $0.25 \mathrm{M}$ sucrose. An aliquot of $100 \mu \mathrm{l}$ was taken from each sample for total protein analysis using BCA assay (Thermo Fisher Scientific, 23227). The samples were stored in $-80^{\circ} \mathrm{C}$ for 7 weeks before lipid extraction.

Lipids of IHH cell samples and lipoprotein fractions were extracted according to Folch at al. [29]. Solvents were evaporated and the lipid extracts immediately dissolved in chloroform/methanol 1:2 (by vol) and right before mass spectrometry $1 \% \mathrm{NH}_{4} \mathrm{OH}$ was added together with SPLASH® LIPIDOMIX® Mass Spec Standard (Avanti Polar Lipids, 330707). The samples were injected into the electrospray source of a triple quadrupole 
mass spectrometer (Agilent 6490 Triple Quad LC/MS with iFunnel Technology; Agilent Technologies, Santa Clara, CA) and their lipids species were identified and quantified using lipid class specific detection modes, as previously described [30]. Retrieved spectra were processed by MassHunter Workstation qualitative analysis software (Agilent Technologies, Inc.), and individual lipid species were quantified using the internal standards and LIMSA software [31].

An aliquot of the lipid extracts were used for determining the FA composition of total lipids of the samples by GC according to the principles described in Käkelä et al. [32]. The extracted lipids were transmethylated by heating with $1 \% \mathrm{H}_{2} \mathrm{SO}_{4}$ in methanol under nitrogen atmosphere, and the formed FA methyl esters (FAME) extracted twice with hexane, dried with anhydrous $\mathrm{Na}_{2} \mathrm{SO}_{4}$ and concentrated. The FA structures were identified using GCMS-QP2010 Ultra (Shimadzu Scientific Instruments, Kyoto, Japan) with mass selective detector (MSD) and the FA proportions quantified using Shimadzu GC-2010 Plus equipment with flame-ionization detector (FID). Both instruments were equipped with Zebron ZB-wax capillary columns $(30 \mathrm{~m}, 0.25 \mathrm{~mm}$ ID and film thickness $0.25 \mu \mathrm{m}$; Phenomenex, Torrence CA, USA).

\subsection{Metabolic labeling of cholesterol ester}

Control IHH and ANGPTL3 KD cells were grown on 6-well plates to confluency. Cells were then incubated with $\left[{ }^{3} \mathrm{H}\right]$ acetic acid $(50 \mu \mathrm{Ci} /$ well; Perkin Elmer, NET003025MC) in medium without puromycin for $3 \mathrm{~h}$, washed with cold PBS and scraped into cold $2 \% \mathrm{NaCl}$. Total lipids were extracted according to Bligh and Dyer [33]. The lipid classes of the samples were separated by thin layer chromatography on silica gels and hexane/diethyl ether/acetic acid $/ \mathrm{H}_{2} \mathrm{O}(65: 15: 1: 0.25, \mathrm{vol} / \mathrm{vol})$ as the solvent system. CE standard was run 
along with the samples, and lipid spots were stained with iodine vapor. CE bands were scraped and their $\left[{ }^{3} \mathrm{H}\right]$ radioactivity was measured by liquid scintillation counting, and the results normalized for total cell protein. Additionally, $\left[{ }^{3} \mathrm{H}\right]$ oleic acid labeling $(2.5 \mu \mathrm{Ci} /$ well; Perkin Elmer, NET289005MC) was carried out as described above for $\left[{ }^{3} \mathrm{H}\right]$ acetic acid.

\subsection{Lipid mediator analysis}

Control IHH and ANGPTL3 KD cells were cultured for $48 \mathrm{~h}$ on 6 -well plates $(n=3)$ in complete Williams E medium with puromycin after which the medium was changed to William's E medium (Gibco, A1217601, no phenol red) with added 10\% (v/v) FBS, 0.2 $\mathrm{mg} / \mathrm{ml}$ glutamine, $100 \mu \mathrm{g} / \mathrm{ml}$ streptomycin and $100 \mathrm{U} / \mathrm{ml}$ penicillin. After $24 \mathrm{~h}$ incubation the medium was changed to one containing $1 \%$ FBS. After $1 \mathrm{~h}$ the plates were frozen and stored in $-80^{\circ} \mathrm{C}$ before analysis. Also a control sample without cells was prepared simultaneously using the same conditions.

Subsequently, two volumes of cold $\mathrm{MeOH}$ containing deuterium-labelled synthetic $\mathrm{d}_{4}$ PGE $2, d_{8}-5-H E T E, d_{4}-L T B_{4}, d_{5}-L X A_{4}, d_{5}-R v D 2, d_{5}-L_{T C}, d_{5}-L^{2} D_{4}, d_{5}-L_{T E}$ (500 pg each), $d_{5}-M a R 1, d_{5}-M a R 2, d_{5}-R v D 3$ (250 pg each), $d_{5}-\operatorname{RvE1}(100 \mathrm{pg})$, and $d_{5}-17 R-R v D 1$ (25 pg) were added onto the wells. Cells were lifted and transferred together with the supernatant. These were then stored at $-20^{\circ} \mathrm{C}$ for at least $45 \mathrm{~min}$ and then centrifuged at $2500 \mathrm{rpm}$ for $10 \mathrm{~min}$. Supernatant was collected and concentrated to $\sim 1.0 \mathrm{ml}$ using a gentle stream of nitrogen gas (TurboVap LV system, Biotage). Solid phase extraction (SPE) was then performed through ExtraHera automated extraction system (Biotage) adding $9 \mathrm{ml}$ of aqueous pH 3.5 HCl solution. The acidified samples were then loaded onto conditioned C18 $500 \mathrm{mg}$ 200-0050-B cartridges (Biotage). Samples were washed with $4.0 \mathrm{ml}$ of $\mathrm{H}_{2} \mathrm{O}$ and $5.0 \mathrm{ml}$ of hexane, and products eluted using $4.0 \mathrm{ml}$ of methyl formate. Solvent was 
evaporated using a gentle stream of nitrogen (TurboVap LV, Biotage) and samples resuspended in $40 \mu \mathrm{l}$ of $\mathrm{MeOH}: \mathrm{H}_{2} \mathrm{O}(1: 1, \mathrm{vol} / \mathrm{vol})$ solution. Samples were centrifuged at 2500 rpm for $5 \mathrm{~min}$ and the supernatant centrifuged again at $9900 \mathrm{rpm}$ for $10 \mathrm{~s}, 4^{\circ} \mathrm{C}$ [34].

LC-MS-MS profiling was performed as described previously [34]. Following C18 SPE and concentration, samples were analyzed using QTrap 6500+ (ABSciex) MS system, coupled with a Shimadzu SIL-20AC HT auto sampler, and LC-20AD LC pumps. Agilent C18 Poroshell column $(150 \mathrm{~mm} \times 4.6 \mathrm{~mm} \times 2.7 \mu \mathrm{m})$ was used to separate lipid mediators. Using a constant flow rate of $0.5 \mathrm{ml} / \mathrm{min}$, eluents gradient started at 20:80:0.01 (vol/vol/vol) in $\mathrm{MeOH} / \mathrm{H}_{2} \mathrm{O} /$ acetic acid for $0.2 \mathrm{~min}$ that was ramped to 50:50:0.01 (vol/vol/vol) over $12 \mathrm{~s}$, maintained for $2 \mathrm{~min}$, ramped to 80:20:0.01 (vol/vol/vol) over $9 \mathrm{~min}$ and maintained for 3.5 $\min$, then ramped to $98: 2: 0.01(\mathrm{vol} / \mathrm{vol} / \mathrm{vol})$ and maintained for $5.5 \mathrm{~min}$. Mediators were identified by matching retention time to synthetic and authentic materials using a MRM developed with signature parent ion and characteristic daughter ions coupled with an Enhanced Product Ion (EPI) for matching mass spectra. Quantification was obtained through calibration curves from synthetic or authentic compounds and deuterium-labeled lipid mediators.

\subsection{Statistical analysis}

For univariate comparisons statistical differences were tested using a two-tailed Student's t-test. Principal Component Analysis (PCA) (Sirius, PRS, Bergen, Norway) was applied for multivariate comparisons of detailed lipid profiles. PCA reveals compositional differences between the samples, and also shows the lipid species mainly responsible for the variation in the data. Data used for the PCA were arcsine transformed to improve data normality and biplots showing the relative positions of the samples and variables were created using 
the first two principal components. The differences between the groups seen in the PCA were further quantitatively analyzed using Soft Independent Modeling of Class Analogy (SIMCA; Sirius) [35]. For lipid mediator statistics multivariate Partial Least Square Discriminant Analysis (PLS-DA) was performed by means of the software SIMCA (Umetrics), building a model with $\mathrm{R} 2 \mathrm{X}=1, \mathrm{R} 2 \mathrm{Y}=1$, and $\mathrm{Q} 2=1$. Variable Importance in Projection (VIP) score was used to highlight the most important lipid mediators discriminating the two groups.

\section{RESULTS AND DISCUSSION}

\subsection{Several genetic pathways related to lipid metabolism are altered in ANGPTL3 knock-down hepatocytes}

ANGPTL3 KD hepatocytes $(\mathrm{IHH})$ were created using an shRNA lentiviral vector. An approximately $90 \% \mathrm{KD}$ efficiency was observed at mRNA level when compared to control cells transduced with non-targeting shRNA (Figure 1A), and the KD effect was evident also at the protein level (Figure 1 B and C). KEGG pathway analysis of the next generation RNA sequencing (RNAseq) data of the ANGPTL3 KD and control IHH cells revealed that several pathways related to lipid metabolism are affected upon the knockdown (Table 1). Similarly, when Reactome pathway analysis was performed using only the statistically significantly up/downregulated genes, all of the ten most affected pathways in the ANGPTL3 KD cells were related to lipid and FA metabolism (Supplementary Figure 1). Thus we chose to study the cells further using lipidomics approaches to address hepatocyte lipid composition and metabolism. 
When looking at the other pathways significantly affected in the KEGG enrichment analysis, two pathways related to longevity emerged and were among the 50 most altered pathways: Longevity regulating pathway - multiple species, and Longevity regulating pathway (Supplementary table 3). This is an interesting and relevant observation when considering that subjects homozygous for LOF variants of ANGPTL3 reach older age than the average population [11]. The Campodimele region, where the ANGPTL3 loss-offunction mutations are found enriched, is known for the longevity of its residents, with the town's population now expected to live to an average age of $95[10 ; 36]$. Moreover, insulin sensitivity is increased in the ANGPTL3 LOF subjects [9]. According to the present KEGG enrichment analysis, also pathways related to insulin resistance/sensitivity, signaling and secretion are altered in the ANGPTL3 KD cells. In a previous study by Tikka et al. [23], it was reported that the KD of ANGPTL3 in IHH improves glucose uptake and that during insulin stimulation the cells secrete TAG-poor VLDL instead of TAG-enriched large VLDL particles. They concluded that silencing of ANGPTL3 improves insulin sensitivity of the $\mathrm{IHH}$ and suggested a liver-specific mechanism to be involved in the insulin-sensitive phenotype of ANGPTL3 LOF subjects. The present RNAseq observations support this notion.

\subsection{The ANGPTL3 knock-down cells are enriched in n-3 and n-6 polyunsaturated and depleted in monounsaturated fatty acids}

As the first lipidomic approach we determined the total FA profile of ANGPTL3 KD and control IHHs by GC. The reported profile represents FAs derived from all lipid species of the cell and the results are presented as molar percentages (Supplementary table 4). In ANGPTL3 KD cells the sum of monounsaturated FAs (MUFAs) was significantly decreased and the sums of both $n-3$ and n-6 polyunsaturated fatty acids (PUFAs) were 
increased when compared to controls (Figure 2A). However, there was no difference in the $n-3 / n-6$ FA ratio or the unsaturated/saturated FA ratio between control and ANGPTL3 KD cells. When the FA data were subjected to principal component analysis (PCA) (Figure 2B), the difference between control and ANGPTL3 KD cell groups reached the statistical significance when tested with SIMCA $(p<0.05)$. The groups separated from each other in the direction of principal component 2 (PC 2), which explained $25.3 \%$ of the total variation. The FAs influencing PC2 the most were 20:5n-3 (eicosapentaenoic acid, EPA) and 20:4n-6 (arachidonic acid, AA) furthest from the origin in the upward direction, and relatively enriched in the ANGPTL3 KD cells, and 20:3n-9 (eicosatrienoic acid) furthest from the origin in the downward direction, and enriched in the controls. EPA and AA can be produced from essential FAs 18:3n-3 (a-linolenic acid) and 18:2n-6 (linoleic acid) via desaturation/elongation processes, respectively, or taken up from the culture medium. FA 20:3n-9 is considered a marker of essential fatty acid deficiency: It is a PUFA synthesized from the non-essential 18:1n-9 (oleic acid) [37]. Of note, we observed in the RNAseq an upregulation of CD36, FABP3, FABP5 and FABP6 mRNAs in the ANGPTL3 KD cells. Since FABP3, -5 and -6 facilitate the cellular uptake of long chain FAs and PUFAs [38-41], we consider it possible that the uptake of FAs and especially PUFAs from the culture medium is enhanced in the ANGPTL3 KD cells.

We next analyzed the IHH lipids at the class level (Supplementary tables 5-8) by mass spectrometry. There were no differences between the control and ANGPTL3 KD cells in the concentrations of the major phospholipid classes PC, PE and PI when normalized to total cellular protein (data not shown). PCA of lipid species profiles in these classes of lipids showed similar separation between control and ANGPTL3 KD cells largely driven by MUFA- (relative reduction in the KD cells) and PUFA- (enrichment in the KD cells) containing lipids as seen in the fatty acid profile (Supplementary Figure 2). PUFAs are 
released from glycerophospholipids through the action of phospholipases such as cytosolic PLA2 (cPLA2) [42;43], two isoforms of which, PLA2G4A and PLA2G4C, are upregulated in our RNAseq data. Cytosolic PLA2 shows specificity for PUFAs, especially for 20:4n-6, 22:6n-3 (docosahexaenoic acid, DHA) and 20:5n-3 [42;43], and plays an important role in the initiation of cellular signaling, hydrolyzing the ester bond at the sn-2 position of glycerophospholipids. Thereby the released PUFAs can be used for the synthesis of bioactive lipid mediators [44]. Phosphatidylinositol (PI) is considered a major source of $20: 4 n-6$ released by cPLA2 since $20: 4 n-6$ is the most abundant PUFA in this PL class, the largest PI component being the 20:4n-6-containing species $38: 4$ (m/z 885 [45;46]. Indeed, in the PCA of our data PI 38:4 was the variable responsible for the largest part of the data variation on PC1 in the leftward direction, and the high level of PI38:4 separated ANGPTL3 KD samples from the control cells. Similarily, the PI 38:5 (likely a mixture of FAs 20:4, 22:5, 20:5 in the sn-2 position) was a characteristic of the ANGPTL3 KD cells.

Interestingly, it has been established that the relative FA compositions of the PI-derived signaling mediators phosphatidylinositol-phosphates (PIPs) reflect that of PI [47] and that polyunsaturated diacylglycerol (DAG) species derived from $\mathrm{PI}(4,5) \mathrm{P}_{2}$ can activate the PKC $\alpha, \varepsilon$, and $\delta$ isoforms [46]. This may also in part explain why the PIP signaling KEGG pathways are significantly affected in the present transcriptomics data (Table 1): The increase of PUFAs in the ANGPTL3 KD cells may through this mechanism impact $\mathrm{PIP}_{2}$ signaling. Closer examination of the KEGG phosphatidylinositol signaling pathway (Supplementary Figure 3) shows that several of its genes are upregulated in the KD cells, but phospholipase C (PLC), a key component in the pathway, is strongly downregulated. This could represent an allostatic mechanism aimed at maintaining a balanced pathway activity. 


\subsection{Knocking down ANGPTL3 changes the lipid mediator profile of hepatocytes}

Prompted by analyses showing changes in several KEGG pathways related to FA and glycerophospholipid metabolism as well as the increase of PUFAs in the ANGPTL3 KD cells, we decided to explore the bioactive PUFA-derived lipid mediators produced by the cells (Supplementary table 9). The results shown represent the sum of intracellular and secreted lipid mediators since both the cells and the medium were analyzed as one sample. There was a clear trend towards an increase of lipid mediators in the ANGPTL3 KD cells, and PLS-DA analysis separated the ANGPTL3 KD and control samples (Figure 3A). Figure 3B displays VIP scores of the 15 most important lipid mediators affecting separation of the groups in the PLS-DA analysis. The mediator with the highest VIP score was RvD6, an important specialized pro-resolving mediator (SPM) with a suggested role in coronary artery disease (CAD). RvD6 was shown to be elevated in CAD patients receiving n-3 FA supplement and it promoted macrophage uptake of blood clots in vitro [48]. Maresin 2 (MaR2) and 22-OH-MaR1, which also showed high VIP scores, are SPMs characterized in macrophages [49] and neutrophils [50], respectively. Of note MaR1, the precursor to 22-OH-MaR1, protects hepatocytes from lipotoxic and hypoxia-induced ER stress [51] suggesting that $22-\mathrm{OH}-\mathrm{MaR} 1$ may display similar biological actions given that this further metabolite retains the potent biological actions of its parent SPM [50]. The protectin pathway products from both n-3 docosapentaenoic acid (DPA) and DHA, i.e. 10S,17S-diHDPA and 10S,17S-diHDHA (protectin DX), were also increased in ANGPTL3 KD cells. PDX prevents lipid-induced ER stress, thereby ameliorating hepatic steatosis and hepatic insulin resistance [52;53]. Another mediator with a high VIP score, 15-epi$\mathrm{LXA}_{4}$ is reported to down-regulate pro-inflammatory eicosanoids and potentiate the production of SPMs [54;55]. 
Prostaglandins (PG) $\mathrm{PGD}_{2}, \mathrm{PGF}_{2 \mathrm{a}}$ and $\mathrm{PGE}_{2}$ all had a high VIP score in the present data. They are generally referred to as pro-inflammatory mediators [56], but especially $\mathrm{PGE}_{2}$ is also important for the lipid mediator class switching at the beginning of the resolution phase as it decreases the production of pro-inflammatory leukotriene $B_{4}\left(L_{T B}\right)$ species [57]. The increased production of lipid mediators in the ANGPTL3 KD cells is consistent with the findings of Holopainen et al. [58], who concluded that elevated amounts of PUFAs (AA, EPA and DHA) in membrane phospholipids lead to increased production of lipid mediators. Increased substrate availability, PLA 2 upregulation, and also the substrate preference of PLA 2 towards PUFA- containing phospholipids [42;43] provide plausible explanations for the observed increase of PUFA-derived lipid mediators in the ANGPTL3 KD cells.

\subsection{Cholesterol ester synthesis is reduced in ANGPTL3 KD cells}

In addition to elucidating the effects of ANGPTL3 KD on phospholipid and FA metabolism, we also investigated cholesterol metabolism by multiple approaches. First, we looked at the profile (Supplementary table 8) and total level (Figure 4A) of cholesterol esters (CEs) in the IHH by using ESI-MS/MS. The relative CE species profile of the ANGPLT3 KD cells showed similar alterations in the MUFA and PUFA-containing species as described for total cellular FAs and phospholipids (Figure 2; Supplementary tables 4-7). The level of the major CE species 18:1 was significantly $(p<0.001)$ reduced and that of the second largest component, the DHA-containing CE 22:6n-3, increased by $74 \%$. The latter finding appears as a class-specific change since DHA was not increased but rather decreased in the total IHH FA profile, driven by altered FA composition of glycerophospholipids. The total level of CE was significantly $(p<0.001)$ decreased in the ANGPTL3 KD cells when compared to controls (Figure 4A). Consistent with this finding, the expression of SOAT1, 
encoding ACAT1, an enzyme synthesizing CEs [59], was decreased in the KD cells both at the mRNA (Figure 4B) and protein (Figure 4C-D) levels. Accordingly, we next investigated CE synthesis and FA incorporation into CEs in the $\mathrm{IHH}$ by $\left[{ }^{3} \mathrm{H}\right]$ acetic acid and $\left[{ }^{3} \mathrm{H}\right]$ oleic acid labeling, respectively, and also analyzed the synthesized unesterified cholesterol in the $\left[{ }^{3} \mathrm{H}\right]$ acetic acid labeled samples. With both radioisotope precursors the level of labeled CEs was significantly $(p<0.001)$ reduced in the ANGPTL3 KD cells (Figure 4E-F), without a change in the labeling of unesterified cholesterol in cells treated with $\left[{ }^{3} \mathrm{H}\right]$ acetic acid (data not shown). Thus, knocking down ANGPTL3 in IHH significantly reduces the esterification of cholesterol. The reduction of CEs putatively mediated by suppression of ACAT1 function might affect the packaging of CEs to nascent VLDL and thereby their secretion. In agreement with these data we previously reported that there was a reduction of $\mathrm{CE} / \mathrm{apoB}$ ratio in plasma VLDL and $\mathrm{LDL}$ particles derived from ANGPTL3 LOF homozygote carriers compared with noncarrier subjects [9;22]. Decreases of VLDL and LDL were observed in pigs treated with avasimibe, an inhibitor of SOAT [60]. Consistently, overexpression of human SOAT1 and 2 in rat hepatoma McA-RH7777 cells was shown to increase CE synthesis and secretion [59]. Another inhibitor of SOATs, pactimibe sulfate, reduced plasma total cholesterol and stabilized atherosclerotic plaques in apolipoprotein E-deficient mice [61].

\subsection{ANGPTL3 deficiency alters the fatty acid profile of lipoproteins}

Since there is no indication of liver disease in ANGPTL3 LOF carriers [11], it is for ethical reasons impossible to obtain liver biopsies from these subjects. We were therefore unable to compare the human LOF mutation carriers' hepatic lipidome to that of the ANGPTL3 KD $\mathrm{IHH}$. However, we analyzed in detail their plasma lipoproteins, the composition of which is determined by both the physiology of hepatocytes secreting VLDL and HDL and the 
modifications mediated by plasma enzymes and lipid transfer proteins. We consequently studied how ANGPTL3 deficiency affects the lipid molecular species profile of lipoproteins, and whether any parallels can be found between the profiles of $\mathrm{IHH}$ subjected to ANGPTL3 KD and lipoproteins of subjects lacking ANGPTL3.

FA analysis of VLDL, LDL and HDL particles isolated from the plasma of ANGPTL3 LOF homozygotes $(n=5)$ and control subjects $(n=10)$ was performed by GC using the same approach as for the $\mathrm{IHH}$. Complete FA profiles of the lipoprotein fractions are found in Supplementary table 10. When inspecting individual FAs, the most prominent difference between the groups was a higher proportion of $18: 2 n-6$ in lipoproteins of the control subjects. The effect remained statistically significant in all lipoprotein fractions. In order to grasp the changes occurring in all the FAs and not merely in the largest components of the profile, we performed a PCA of the entire data using standardized mol\% values. A clear separation of the groups can be seen in the PCA biplot (Figure 5). PC1 explains $31.7 \%$ of the total variation and separates the different fractions (VLDL on the right, LDL in the middle and HDL on the left), whereas PC2 explains $21.3 \%$ of the variation and separates the control subjects (at the bottom) and the ANGPTL3 LOF homozygotes (at the top). The VLDL fractions contained relatively more MUFAs and FAs having <18-carbons, while the HDL fractions were enriched in 20-22-carbon PUFAs, >20-24-carbon SFAs or MUFAs (ANGPTL3 LOF homozygotes) and saturated dimethyl acetals derived from plasmalogens (controls). The right to left change (PC1) in the quality of the FAs is likely affected by the activity of LPL, which is higher in ANGPTL3 LOF homozygotes [9]. The efficiency of LPLmediated hydrolysis of ester bonds decreases with increasing chain length and unsaturation [62;63], and thus relatively short SFAs would be hydrolyzed first, followed by MUFAs, leaving the longest PUFAs to be hydrolyzed last. Importantly the FA profile of the different lipoprotein fractions is not influenced by the action of two lipid transfer proteins, 
CETP and PLTP, since no significant differences in their activities were observed between homozygous ANGPTL3 LOF variant carriers and control subjects [27]. We think that the impact of diet can be ruled out, as the comparison of dietary intakes between ANGPTL3 LOF subjects and controls did not reveal appreciable differences (data not shown).

\subsection{PUFA-containing TAGs are relatively increased in the lipoproteins of ANGPTL3 LOF homozygotes}

We next analyzed the lipid components of lipoprotein particles derived from ANGPTL3 LOF homozygotes and control subjects by ESI-MS/MS. The lipoproteins of the ANGPTL3 LOF homozygotes have been reported to contain less TAG and CEs when compared to those of control subjects [9]; Our results are consistent with these findings (fold changes of lipids at the class level are listed in Supplementary table 11 and species profiles of each class in Supplementary tables 12-16). At the species level the findings in CEs and TAGs were, however, opposite to each other. Compared to the controls, the CEs in the lipoproteins of ANGPTL3 LOF homozygous subjects included larger proportions of the species with 16:1 and 18:1 FAs (Supplementary table 13), whereas in TAGs the relative levels of PUFA-containing species were elevated (Figure 6, Supplementary table 12). The latter result is likely due to the increased activity of LPL in ANGPTL3 LOF homozygotes, since long PUFAs are poor substrates for LPL [62;63], and are thus retained. Interestingly, $\mathrm{Xu}$ et al. [20] found that knocking down ANGPTL3 in HuH7 cells increased the cellular concentration of long-chain TAGs, so it is possible that TAGs in VLDL particles of ANGPTL3-deficient subjects could be somewhat enriched in PUFAs already as they are secreted. The cellular concentrations of TAGs in the IHH model employed here were unfortunately too low to be reliably profiled at species level. Thus, we 
are unable to draw conclusions on the relationship of TAG molecular species composition in hepatocytes and plasma lipoproteins.

CEs are not substrates of LPL, since LPL is a serine-histidine hydrolase and its active site favors especially oxyester bond on the glycerol backbone [64-66]. Thus, CEs are not affected by the increased activity of LPL but another, unknown mechanism must modify their composition in the LOF mutation carriers. One putative explanation for these CE species shown in lipoproteins is altered substrate specificity of LCAT. Most of the CEs in

human plasma lipoproteins are derived from the function of LCAT [67]. Although LCAT displays specificity for the sn-2 position of PC, the composition of the CEs in human plasma does not match that of the sn-2 acyl group [68]. We can speculate that ANGPTL3 deficiency creates changes in FA distribution in PC molecules and therefore LCAT FA specificity or the positional specificity in PC [69] is altered and leads to generation of the CE species shown in the present study.

\subsection{ANGPTL3 deficiency changes the quality of SM and its ratio to PC}

The surface monolayer of lipoprotein particles consists mainly of PCs, sphingomyelins (SMs) and lysoPCs. We found intriguing changes between ANGPTL3 LOF subjects and controls in the species profiles of SM, PC and lysoPC in all the lipoprotein fractions (Figure 7A-C, Supplementary tables 14-16). SMs of the lipoproteins obtained from ANGPTL3 LOF homozygous subjects contained relatively more of the long SM species, especially $24: 1$ and $24: 2$, and less of short saturated SMs than the controls. The changes in lysoPCs were similar to those seen in TAGs (enrichment of PUFAs) and could be explained by the increased LPL-facilitated PLA1 activity shown to be able to release the 
FA in the sn-1 position of PC[70]. The study by McLean et al. [70] also indicated that the longer the FA in the sn-2 position, the higher activity LPL has against the sn-1 FA, resulting in lysoPCs enriched in sn-2 long chain FAs. Moreover, in the lipoproteins of ANGPLT3 LOF homozygous subjects there was an enrichment of alkyl ether PCs (Supplementary table 16). Since these species are synthesized in peroxisomes [71], the finding could imply that the peroxisomes of the LOF subjects are more active. However, to our knowledge nothing has been reported on the peroxisomal function in ANGPTL3 LOF mutation carriers. Another plausible explanation for the increase of alkyl ether PCs in the LOF lipoproteins is that LPL and EL, which are subject to regulation by ANGPTL3, are only hydrolyzing ester and not ether bonds [64;65;72]. Thereby, the elevated phopholipase activity of these enzymes in LOF subjects could result in a relative enrichment of nonsubstrate ether phospholipid species.

The SM/PC ratio was increased in all lipoprotein fractions derived from ANGPTL3 LOF homozygous subjects when compared to controls (Figure 7A). This could result from several different mechanisms. We did not see changes in the SM/PC ratio in the $\mathrm{IHH}$ cell model (data not shown). However, this does not rule out the possibility that nascent VLDLs may be enriched in SM; Once entering general circulation these particles could be rapidly lipolyzed by LPL, and the extra surface SM together with some PCs could be transported by PLTP to HDL and LDL, therefore increasing the proportion of SM in these particles. Indeed, it has been shown that SM is very efficiently transferred by PLTP [73].

The enrichment of SMs in lipoproteins obtained from ANGPTL3 LOF homozygotes is highly interesting in the light of the study of Ruuth et al. [74], where the saturated SM species and SM 16:1 were associated with LDL aggregation, which increases the risk of cardiovascular death. Thus, even though the SM/PC ratio is increased in the LDL of ANGPTL3 LOF homozygotes, the quality of SM (elevation of 24:1 and 24:2 species) in 
these subjects could make their LDL less prone to aggregation as compared to controls. It should also be noted that in HDLs an increased SM/PC ratio leads to an increased ability to receive cholesterol from cells, improving the cardioprotective capacity of the lipoprotein [75]. On the other hand, we have to consider that enrichment of HDL with SM inhibits cholesterol esterification, and this inhibition can be reversed by the degradation of SM, which demonstrates that $\mathrm{SM}$ is a physiological inhibitor of cholesterol esterification in circulation and competes with the PC acyl donor on the surface of HDL particle [76]. This would attenuate reverse cholesterol transport process which needs LCAT facilitated step of free cholesterol esterification. When interpreting the above data, one should also keep in mind that the absolute amounts of cholesterol and TAGs in the lipoproteins of ANGPTL3 deficient subjects are strongly reduced, which is by itself beneficial for the cardiovascular health of these individuals.

\section{CONCLUSIONS}

Loss-of-function mutations in ANGPTL3, an inhibitor of lipoprotein lipase (LPL), cause a drastic reduction of all major serum lipoproteins and are protective against the development of atherosclerotic CVD. Therefore, ANGPTL3 is emerging as a therapy target; Clinical trials with agents targeting ANGPTL3 have already reached phase 3. Although ANGPTL3 is produced almost exclusively by the liver, there is no data on its function in determining the hepatocellular gene expression patterns and lipidome. In the present study we characterized the impacts of ANGPTL3 depletion on the hepatocyte $(\mathrm{IHH})$ transcriptome and lipidome and whether there are parallels between the lipidomes of ANGPTL3-deficient hepatocytes and of plasma VLDL, LDL, and HDL isolated from homozygous ANGPTL3 LOF mutation carriers. 
$\mathrm{IHH}$ depleted of ANGPTL3 displayed distinct changes in total FA composition and in the molecular species composition of several lipid classes, characterized by an increase in the mol\% of $n-6$ and $n-3$ polyunsaturated FAs (PUFAs). The VLDL, LDL and HDL of the ANGPTL3 LOF subjects showed a significant drop of 18:2n-6, while several polyunsaturated TAG species were enriched, most likely due to elevated activity of LPL which prefers saturated and shorter chain FAs esterified to the glycerol backbone [62;63]. The LOF subjects' lipoproteins displayed an increased SM/PC molar ratio and enrichment of 24:1 and 24:2 SM species. Since the SM/PC ratio is increased in all lipoprotein fractions of $\mathrm{t}$ LOF subjects, it cardiometabolic implications are controversial. Similar to the $\mathrm{IHH}$, increased mol\% of PUFAs was found in LysoPCs, which could result from increased PLA1 activity of LPL [70].

Cholesterol esters were markedly reduced in the ANGPTL3 KD hepatocytes, coinciding with suppression of SOAT1/ACAT1. However, we did not see any change in the expression of SOAT2, which is the major isoform in human liver in vivo [77]. Previously, we have also detected a low CE/apoB100 molar ratio in the ANGPTL3 LOF homozygote subjects [9] as well as reduced CEs in VLDL remnants of these subjects [22]. However, the putative causality between the cultured hepatocyte and in vivo observations requires further investigation.

The PUFA increase in the ANGPTL3-depleted IHH coincided with an elevation of PUFAderived lipid mediators. Among these there were several mediators with documented functions in resolution of inflammation, protection from lipotoxic and hypoxia-induced ER stress, hepatic steatosis and insulin resistance $[52 ; 53]$ or in mediating the recovery from cardiovascular events [48;55]. Notably, the resolution of inflammation has emerged as a 
crucial physiological mechanism affecting atherogenesis [78-80]. Consistent with the lipid mediator data, altered expression of several KEGG pathways related to fatty acid metabolism was observed. The impact of ANGPTL3 inhibition on synthesis of the PUFAderived mediators deserves further investigation; when fresh plasma becomes available from the ANGPTL3 LOF carriers, it will be extremely interesting to study the lipid mediators in these subjects.

To conclude, the present work reveals distinct impacts of ANGPTL3 depletion on the hepatocellular lipidome, transcriptome and lipid mediators, as well as on the lipidome of lipoproteins isolated from the plasma of ANGPTL3-deficient human subjects. This lipidomic/transcriptomic data is important to consider when targeting ANGPTL3 for therapy and translating it to the human context.

\section{Acknowledgements}

We thank all the volunteers who participated in this study. We also thank Meghana Nagaraj, MSc, as well as Riikka Kosonen, MSc, and Sanna Sihvo for the technical assistance and Anna Montali, BSc, for her assistance in recruiting study subjects.

\section{Funding}

Novo Nordisk Foundation (V.M. Olkkonen), the Paavo Nurmi Foundation (V.M. Olkkonen), the Liv och Hälsa Foundation (V.M. Olkkonen), the Finnish Foundation for Cardiovascular Research (M. Jauhiainen, V.M. Olkkonen), the Jane and Aatos Erkko Foundation (M. Jauhiainen), the Magnus Ehrnrooth Foundation (M. Jauhiainen), and Progetto Ateneo 2006 and Progetto Ateneo 2011 from Sapienza University of Rome (M. Arca) are 
acknowledged for financial support. J.D is funded by European Research Council (ERC) under the European Union's Horizon 2020 research and innovation programme (grant no: 677542) and the Barts Charity (grant no: MGU0343) to J.D. J.D. is also supported by a Sir Henry Dale Fellowship jointly funded by the Wellcome Trust and the Royal Society (grant $107613 / Z / 15 / Z)$

\section{Conflicts of interest}

None.

\section{REFERENCES}

[1] WHO, 2018. World Health Organization Fact Sheet: The Top 10 Causes of Death 2019. https://www.who.int/news-room/fact-sheets/detail/the-top-10-causes-of-death. 24 May 2018 (accessed 2 January 2020).

[2] Stone, N.J., Robinson, J.G., Lichtenstein, A.H., Bairey Merz, C.N., Blum, C.B., Eckel, R.H., et al., 2014. 2013 ACC/AHA guideline on the treatment of blood cholesterol to reduce atherosclerotic cardiovascular risk in adults: a report of the American College of Cardiology/American Heart Association Task Force on Practice Guidelines. Circulation 129:S1-45.

[3] Hegele, R.A., Tsimikas, S., 2019. Lipid-Lowering Agents. Circulation research 124:386-404.

[4] Köster, A., Chao, Y.B., Mosior, M., Ford, A., Gonzalez-DeWhitt, P.A., Hale, J.E., et al., 2005. Transgenic angiopoietin-like (angptl)4 overexpression and targeted disruption of angpt/4 and angpt|3: regulation of triglyceride metabolism. Endocrinology 146:4943-4950.

[5] Gusarova, V., Banfi, S., Alexa-Braun, C.A., Shihanian, L.M., Mintah, I.J., Lee, J.S., et al., 2017. ANGPTL8 Blockade With a Monoclonal Antibody Promotes Triglyceride Clearance, Energy Expenditure, and Weight Loss in Mice. Endocrinology 158:1252-1259. 
[6] Musunuru, K., Pirruccello, J.P., Do, R., Peloso, G.M., Guiducci, C., Sougnez, C., et al., 2010. Exome sequencing, ANGPTL3 mutations, and familial combined hypolipidemia. The New England Journal of Medicine 363:2220-2227.

[7] Stitziel, N.O., Khera, A.V., Wang, X., Bierhals, A.J., Vourakis, A.C., Sperry, A.E., et al., 2017. ANGPTL3 Deficiency and Protection Against Coronary Artery Disease. Journal of the American College of Cardiology 69:2054-2063.

[8] Minicocci, I., Santini, S., Cantisani, V., Stitziel, N., Kathiresan, S., Arroyo, J.A., et al., 2013. Clinical characteristics and plasma lipids in subjects with familial combined hypolipidemia: a pooled analysis. Journal of Lipid Research 54:3481-3490.

[9] Robciuc, M.R., Maranghi, M., Lahikainen, A., Rader, D., Bensadoun, A., Oorni, K., et al., 2013. Angptl3 deficiency is associated with increased insulin sensitivity, lipoprotein lipase activity, and decreased serum free fatty acids. Arteriosclerosis, Thrombosis, and Vascular Biology 33:17061713.

[10] Lawson, T., 2011. A Year in the Village of Eternity: the Lifestyle of Longevity in Campodimele, Italy. 1st ed. New York: Bloomsbury.

[11] Minicocci, I., Montali, A., Robciuc, M.R., Quagliarini, F., Censi, V., Labbadia, G., et al., 2012. Mutations in the ANGPTL3 gene and familial combined hypolipidemia: a clinical and biochemical characterization. The Journal of Clinical Endocrinology and Metabolism 97:E1266-75.

[12] Dewey, F.E., Gusarova, V., Dunbar, R.L., O'Dushlaine, C., Schurmann, C., Gottesman, O., et al., 2017. Genetic and Pharmacologic Inactivation of ANGPTL3 and Cardiovascular Disease. The New England Journal of Medicine 377:211-221.

[13] Gusarova, V., Alexa, C.A., Wang, Y., Rafique, A., Kim, J.H., Buckler, D., et al., 2015. ANGPTL3 blockade with a human monoclonal antibody reduces plasma lipids in dyslipidemic mice and monkeys. Journal of Lipid Research 56:1308-1317. 
[14] Graham, M.J., Lee, R.G., Brandt, T.A., Tai, L.J., Fu, W., Peralta, R., et al., 2017. Cardiovascular and Metabolic Effects of ANGPTL3 Antisense Oligonucleotides. The New England Journal of Medicine 377:222-232.

[15] Romeo, S., Yin, W., Kozlitina, J., Pennacchio, L.A., Boerwinkle, E., Hobbs, H.H., et al., 2009. Rare loss-of-function mutations in ANGPTL family members contribute to plasma triglyceride levels in humans. The Journal of Clinical Investigation 119:70-79.

[16] Nidhina Haridas, P.A., Soronen, J., Sädevirta, S., Mysore, R., Quagliarini, F., Pasternack, A., et al., 2015. Regulation of Angiopoietin-Like Proteins (ANGPTLs) 3 and 8 by Insulin. The Journal of Clinical Endocrinology and Metabolism 100:E1299-307.

[17] Shimizugawa, T., Ono, M., Shimamura, M., Yoshida, K., Ando, Y., Koishi, R., et al., 2002. ANGPTL3 decreases very low density lipoprotein triglyceride clearance by inhibition of lipoprotein lipase. The Journal of Biological Chemistry 277:33742-33748.

[18] Shimamura, M., Matsuda, M., Yasumo, H., Okazaki, M., Fujimoto, K., Kono, K., et al., 2007. Angiopoietin-like protein3 regulates plasma HDL cholesterol through suppression of endothelial lipase. Arteriosclerosis, Thrombosis, and Vascular Biology 27:366-372.

[19] Wang, Y., Gusarova, V., Banfi, S., Gromada, J., Cohen, J.C., Hobbs, H.H., 2015. Inactivation of ANGPTL3 reduces hepatic VLDL-triglyceride secretion. Journal of Lipid Research 56:12961307.

[20] Xu, Y.X., Redon, V., Yu, H., Querbes, W., Pirruccello, J., Liebow, A., et al., 2018. Role of angiopoietin-like 3 (ANGPTL3) in regulating plasma level of low-density lipoprotein cholesterol. Atherosclerosis 268:196-206.

[21] Shimamura, M., Matsuda, M., Kobayashi, S., Ando, Y., Ono, M., Koishi, R., et al., 2003. Angiopoietin-like protein 3, a hepatic secretory factor, activates lipolysis in adipocytes. Biochemical and Biophysical Research Communications 301:604-609. 
[22] Tikkanen, E., Minicocci, I., Hällfors, J., Di Costanzo, A., D'Erasmo, L., Poggiogalle, E., et al., 2019. Metabolomic Signature of Angiopoietin-Like Protein 3 Deficiency in Fasting and Postprandial State. Arteriosclerosis, Thrombosis, and Vascular Biology 39:665-674.

[23] Tikka, A., Soronen, J., Laurila, P.P., Metso, J., Ehnholm, C., Jauhiainen, M., 2014. Silencing of ANGPTL 3 (angiopoietin-like protein 3) in human hepatocytes results in decreased expression of gluconeogenic genes and reduced triacylglycerol-rich VLDL secretion upon insulin stimulation. Bioscience Reports 34:e00160.

[24] Yu, G., Wang, L.G., Han, Y., He, Q.Y., 2012. clusterProfiler: an R package for comparing biological themes among gene clusters. Omics : a journal of integrative biology 16:284-287.

[25] Csárdi, G., Nepusz, T., 2006. The igraph software package for complex network research. InterJournal Complex Systems 1695:1-9.

[26] Shannon, P., Markiel, A., Ozier, O., Baliga, N.S., Wang, J.T., Ramage, D., et al., 2003. Cytoscape: a software environment for integrated models of biomolecular interaction networks. Genome Research 13:2498-2504.

[27] Minicocci, I., Tikka, A., Poggiogalle, E., Metso, J., Montali, A., Ceci, F., et al., 2016. Effects of angiopoietin-like protein 3 deficiency on postprandial lipid and lipoprotein metabolism. Journal of Lipid Research 57:1097-1107.

[28] Hilvo, M., Simolin, H., Metso, J., Ruuth, M., Öörni, K., Jauhiainen, M., et al., 2018. PCSK9 inhibition alters the lipidome of plasma and lipoprotein fractions. Atherosclerosis 269:159-165.

[29] Folch, J., Lees, M., Sloane Stanley, G.H., 1957. A simple method for the isolation and purification of total lipides from animal tissues. The Journal of Biological Chemistry 226:497-509.

[30] Ruhanen, H., Nidhina Haridas, P.A., Eskelinen, E.L., Eriksson, O., Olkkonen, V.M., Käkelä, R., 2017. Depletion of TM6SF2 disturbs membrane lipid composition and dynamics in $\mathrm{HuH} 7$ hepatoma cells. Biochimica et Biophysica Acta - Molecular and Cell Biology of Lipids 1862:676-685.

[31] Haimi, P., Uphoff, A., Hermansson, M., Somerharju, P., 2006. Software tools for analysis of mass spectrometric lipidome data. Analytical Chemistry 78:8324-8331. 
[32] Käkelä, R., Käkelä, A., Kahle, S., Becker, B.H., Kelly, A., Furness, R.W., 2005. Fatty acid signatures in plasma of captive herring gulls as indicators of demersal or pelagic fish diet. Marine Ecology Progress Series. 293:191-200.

[33] Bligh, E.G., Dyer, W.J., 1959. A rapid method of total lipid extraction and purification. Canadian Journal of Biochemistry and Physiology 37:911.

[34] Dalli, J., Colas, R.A., Walker, M.E., Serhan, C.N., 2018. Lipid Mediator Metabolomics Via LCMS/MS Profiling and Analysis. Methods in molecular biology (Clifton, N.J.) 1730:59-72.

[35] Wold, S., Sjöström, M., 1977. SIMCA: A Method for Analyzing Chemical Data in Terms of Similarity and Analogy. In: Kowalski, B., editor. Chemometrics: Theory and Application, Washington D.C.: American Chemical Society, p. 243-282.

[36] Cugini, P., Leone, G., Lucia, P., Sepe, F.A., Pelosio, A., Caparelli, T., et al., 1994. Campodimele study: blood pressure and heart rate pattern in clinically healthy elderly subjects. Chronobiology International 11:381-392.

[37] Ichi, I., Kono, N., Arita, Y., Haga, S., Arisawa, K., Yamano, M., et al., 2014. Identification of genes and pathways involved in the synthesis of Mead acid (20:3n-9), an indicator of essential fatty acid deficiency. Biochimica et Biophysica Acta - Molecular and Cell Biology of Lipids 1841:204-213.

[38] Kane, C.D., Coe, N.R., Vanlandingham, B., Krieg, P., Bernlohr, D.A., 1996. Expression, purification, and ligand-binding analysis of recombinant keratinocyte lipid-binding protein (MAL-1), an intracellular lipid-binding found overexpressed in neoplastic skin cells. Biochemistry 35:28942900.

[39] Murphy, E.J., Owada, Y., Kitanaka, N., Kondo, H., Glatz, J.F., 2005. Brain arachidonic acid incorporation is decreased in heart fatty acid binding protein gene-ablated mice. Biochemistry 44:6350-6360. 
[40] Ehehalt, R., Sparla, R., Kulaksiz, H., Herrmann, T., Fullekrug, J., Stremmel, W., 2008. Uptake of long chain fatty acids is regulated by dynamic interaction of FAT/CD36 with cholesterol/sphingolipid enriched microdomains (lipid rafts). BMC Cell Biology 9:45-2121-9-45.

[41] Islam, A., Kagawa, Y., Sharifi, K., Ebrahimi, M., Miyazaki, H., Yasumoto, Y., et al., 2014. Fatty Acid Binding Protein 3 Is Involved in $n-3$ and $n-6$ PUFA transport in mouse trophoblasts. The Journal of Nutrition 144:1509-1516.

[42] Shikano, M., Masuzawa, Y., Yazawa, K., Takayama, K., Kudo, I., Inoue, K., 1994. Complete discrimination of docosahexaenoate from arachidonate by $85 \mathrm{kDa}$ cytosolic phospholipase A2 during the hydrolysis of diacyl- and alkenylacylglycerophosphoethanolamine. Biochimica et Biophysica Acta - Lipids and Lipid Metabolism 1212:211-216.

[43] Batchu, K.C., Hänninen, S., Jha, S.K., Jeltsch, M., Somerharju, P., 2016. Factors regulating the substrate specificity of cytosolic phospholipase A2-alpha in vitro. Biochimica et Biophysica Acta - Molecular and Cell Biology of Lipids 1861:1597-1604.

[44] Norris, P.C., Gosselin, D., Reichart, D., Glass, C.K., Dennis, E.A., 2014. Phospholipase A2 regulates eicosanoid class switching during inflammasome activation. Proceedings of the National Academy of Sciences of the United States of America 111:12746-12751.

[45] Hicks, A.M., DeLong, C.J., Thomas, M.J., Samuel, M., Cui, Z., 2006. Unique molecular signatures of glycerophospholipid species in different rat tissues analyzed by tandem mass spectrometry. Biochimica et Biophysica Acta - Molecular and Cell Biology of Lipids 1761:10221029.

[46] D'Souza, K., Epand, R.M., 2014. Enrichment of phosphatidylinositols with specific acyl chains. Biochimica et Biophysica Acta - Biomembranes 1838:1501-1508.

[47] Traynor-Kaplan, A., Kruse, M., Dickson, E.J., Dai, G., Vivas, O., Yu, H., et al., 2017. Fatty-acyl chain profiles of cellular phosphoinositides. Biochimica et Biophysica Acta - Molecular and Cell Biology of Lipids 1862:513-522. 
[48] Elajami, T.K., Colas, R.A., Dalli, J., Chiang, N., Serhan, C.N., Welty, F.K., 2016. Specialized proresolving lipid mediators in patients with coronary artery disease and their potential for clot remodeling. FASEB journal: official publication of the Federation of American Societies for Experimental Biology 30:2792-2801.

[49] Deng, B., Wang, C.W., Arnardottir, H.H., Li, Y., Cheng, C.Y., Dalli, J., et al., 2014. Maresin biosynthesis and identification of maresin 2, a new anti-inflammatory and pro-resolving mediator from human macrophages. PloS one 9:e102362.

[50] Colas, R.A., Dalli, J., Chiang, N., Vlasakov, I., Sanger, J.M., Riley, I.R., et al., 2016. Identification and Actions of the Maresin 1 Metabolome in Infectious Inflammation. Journal of Immunology (Baltimore, Md.: 1950) 197:4444-4452.

[51] Rius, B., Duran-Guell, M., Flores-Costa, R., Lopez-Vicario, C., Lopategi, A., Alcaraz-Quiles, J., et al., 2017. The specialized proresolving lipid mediator maresin 1 protects hepatocytes from lipotoxic and hypoxia-induced endoplasmic reticulum stress. FASEB journal: official publication of the Federation of American Societies for Experimental Biology 31:5384-5398.

[52] Jung, T.W., Kyung, E.J., Kim, H.C., Shin, Y.K., Lee, S.H., Park, E.S., et al., 2018. Protectin DX Ameliorates Hepatic Steatosis by Suppression of Endoplasmic Reticulum Stress via AMPKInduced ORP150 Expression. The Journal of Pharmacology and Experimental Therapeutics 365:485-493.

[53] Jung, T.W., Ahn, S.H., Shin, J.W., Kim, H.C., Park, E.S., Abd El-Aty, A.M., et al., 2019. Protectin DX ameliorates palmitate-induced hepatic insulin resistance through AMPK/SIRT1mediated modulation of fetuin-A and SeP expression. Clinical and Experimental Pharmacology \& Physiology 46:898-909.

[54] Dakin, S.G., Colas, R.A., Newton, J., Gwilym, S., Jones, N., Reid, H.A.B., et al., 2019. 15-EpiLXA4 and MaR1 counter inflammation in stromal cells from patients with Achilles tendinopathy and rupture. FASEB journal: official publication of the Federation of American Societies for Experimental Biology 33:8043-8054. 
[55] Kain, V., Liu, F., Kozlovskaya, V., Ingle, K.A., Bolisetty, S., Agarwal, A., et al., 2017. Resolution Agonist 15-epi-Lipoxin A4 Programs Early Activation of Resolving Phase in PostMyocardial Infarction Healing. Scientific Reports 7:9999-017-10441-8.

[56] Ricciotti, E., FitzGerald, G.A., 2011. Prostaglandins and inflammation. Arteriosclerosis, Thrombosis, and Vascular Biology 31:986-1000.

[57] Levy, B.D., Clish, C.B., Schmidt, B., Gronert, K., Serhan, C.N., 2001. Lipid mediator class switching during acute inflammation: signals in resolution. Nature Immunology 2:612-619.

[58] Holopainen, M., Colas, R.A., Valkonen, S., Tigistu-Sahle, F., Hyvärinen, K., Mazzacuva, F., et al., 2019. Polyunsaturated fatty acids modify the extracellular vesicle membranes and increase the production of proresolving lipid mediators of human mesenchymal stromal cells. Biochimica et Biophysica Acta - Molecular and Cell Biology of Lipids 1864:1350-1362.

[59] Liang, J.J., Oelkers, P., Guo, C., Chu, P.C., Dixon, J.L., Ginsberg, H.N., et al., 2004. Overexpression of human diacylglycerol acyltransferase 1, acyl-coa:cholesterol acyltransferase 1, or acyl-CoA:cholesterol acyltransferase 2 stimulates secretion of apolipoprotein B-containing lipoproteins in McA-RH7777 cells. The Journal of Biological Chemistry 279:44938-44944.

[60] Burnett, J.R., Wilcox, L.J., Telford, D.E., Kleinstiver, S.J., Barrett, P.H., Newton, R.S., et al., 1999. Inhibition of ACAT by avasimibe decreases both VLDL and LDL apolipoprotein B production in miniature pigs. Journal of Lipid Research 40:1317-1327.

[61] Terasaka, N., Miyazaki, A., Kasanuki, N., Ito, K., Ubukata, N., Koieyama, T., et al., 2007. ACAT inhibitor pactimibe sulfate (CS-505) reduces and stabilizes atherosclerotic lesions by cholesterol-lowering and direct effects in apolipoprotein E-deficient mice. Atherosclerosis 190:239247.

[62] Wang, C.S., Bass, H., Whitmer, R., McConathy, W.J., 1993. Effects of albumin and apolipoprotein C-II on the acyl-chain specificity of lipoprotein lipase catalysis. Journal of Lipid Research 34:2091-2098. 
[63] Sato, K., Takahashi, T., Takahashi, Y., Shiono, H., Katoh, N., Akiba, Y., 1999. Preparation of chylomicrons and VLDL with monoacid-rich triacylglycerol and characterization of kinetic parameters in lipoprotein lipase-mediated hydrolysis in chickens. The Journal of Nutrition 129:126131.

[64] McLean, L.R., Demel, R.A., Socorro, L., Shinomiya, M., Jackson, R.L., 1986. Mechanism of action of lipoprotein lipase. Methods in Enzymology 129:738-763.

[65] Olivecrona, T., Bengtsson-Olivecrona, G., 1987. Lipoprotein lipase from milk - The model enzyme in lipoprotein lipase research. In: Borensztajn, J., editor. Lipoprotein lipase, Chicago: Evener Publishers, p. 15-58.

[66] Dugi, K.A., Dichek, H.L., Talley, G.D., Brewer, H.B.,Jr, Santamarina-Fojo, S., 1992. Human lipoprotein lipase: the loop covering the catalytic site is essential for interaction with lipid substrates. The Journal of Biological Chemistry 267:25086-25091.

[67] Glomset, J.A., 1979. Lecithin: cholesterol acyltransferase. An exercise in comparative biology. Progress in Biochemical Pharmacology 15:41-66.

[68] Liu, M., Bagdade, J.D., Subbaiah, P.V., 1995. Specificity of lecithin:cholesterol acyltransferase and atherogenic risk: comparative studies on the plasma composition and in vitro synthesis of cholesteryl esters in 14 vertebrate species. Journal of Lipid Research 36:1813-1824.

[69] Subbaiah, P.V., Sowa, J.M., Davidson, M.H., 2004. Evidence for altered positional specificity of LCAT in vivo: studies with docosahexaenoic acid feeding in humans. Journal of Lipid Research $45: 2245-2251$.

[70] McLean, L.R., Best, S., Balasubramaniam, A., Jackson, R.L., 1986. Fatty acyl chain specificity of phosphatidylcholine hydrolysis catalyzed by lipoprotein lipase. Effect of apolipoprotein C-II and its (56-79) synthetic fragment. Biochimica et Biophysica Acta - Lipids and Lipid Metabolism 878:446-449.

[71] van den Bosch, H., Schrakamp, G., Hardeman, D., Zomer, A.W., Wanders, R.J., Schutgens, R.B., 1993. Ether lipid synthesis and its deficiency in peroxisomal disorders. Biochimie 75:183-189. 
[72] Griffon, N., Budreck, E.C., Long, C.J., Broedl, U.C., Marchadier, D.H., Glick, J.M., et al., 2006. Substrate specificity of lipoprotein lipase and endothelial lipase: studies of lid chimeras. Journal of Lipid Research 47:1803-1811.

[73] Huuskonen, J., Olkkonen, V.M., Jauhiainen, M., Metso, J., Somerharju, P., Ehnholm, C., 1996. Acyl chain and headgroup specificity of human plasma phospholipid transfer protein. Biochimica et Biophysica Acta - Lipids and Lipid Metabolism 1303:207-214.

[74] Ruuth, M., Nguyen, S.D., Vihervaara, T., Hilvo, M., Laajala, T.D., Kondadi, P.K., et al., 2018. Susceptibility of low-density lipoprotein particles to aggregate depends on particle lipidome, is modifiable, and associates with future cardiovascular deaths. European Heart Journal 39:25622573.

[75] Horter, M.J., Sondermann, S., Reinecke, H., Bogdanski, J., Woltering, A., Kerber, S., et al., 2002. Associations of HDL phospholipids and paraoxonase activity with coronary heart disease in postmenopausal women. Acta Physiologica Scandinavica 176:123-130.

[76] Subbaiah, P.V., Liu, M., 1993. Role of sphingomyelin in the regulation of cholesterol esterification in the plasma lipoproteins. Inhibition of lecithin-cholesterol acyltransferase reaction. The Journal of Biological Chemistry 268:20156-20163.

[77] Parini, P., Davis, M., Lada, A.T., Erickson, S.K., Wright, T.L., Gustafsson, U., et al., 2004. ACAT2 is localized to hepatocytes and is the major cholesterol-esterifying enzyme in human liver. Circulation 110:2017-2023.

[78] Akagi, D., Chen, M., Toy, R., Chatterjee, A., Conte, M.S., 2015. Systemic delivery of proresolving lipid mediators resolvin D2 and maresin 1 attenuates intimal hyperplasia in mice. FASEB journal: official publication of the Federation of American Societies for Experimental Biology 29:2504-2513.

[79] Fredman, G., Hellmann, J., Proto, J.D., Kuriakose, G., Colas, R.A., Dorweiler, B., et al., 2016. An imbalance between specialized pro-resolving lipid mediators and pro-inflammatory leukotrienes promotes instability of atherosclerotic plaques. Nature Communications 7:12859. 
[80] Gerlach, B.D., Marinello, M., Heinz, J., Rymut, N., Sansbury, B.E., Riley, C.O., et al., 2020. Resolvin D1 promotes the targeting and clearance of necroptotic cells. Cell Death \& differentiation 27:525-539. 
Table 1. Several KEGG pathways related to lipid metabolism are altered in ANGPLT3 knock-down hepatocytes. A total of 171 pathways were obtained in the KEGG pathway enrichment analysis, and a full list of pathways and statistical values is available as Supplementary table 3.

\begin{tabular}{llll}
$\begin{array}{l}\text { Ranking } \\
(/ \mathbf{1 7 1})\end{array}$ & Pathway & p value & $\begin{array}{l}\text { Adjusted } \\
\text { p value }\end{array}$ \\
\hline $\mathbf{1}$ & Inositol phosphate metabolism & $2.329 \mathrm{E}-25$ & $7.196 \mathrm{E}-23$ \\
$\mathbf{2}$ & Phosphatidylinositol signaling system & $1.581 \mathrm{E}-23$ & $2.442 \mathrm{E}-21$ \\
$\mathbf{3}$ & Fatty acid metabolism & $1.198 \mathrm{E}-22$ & $1.234 \mathrm{E}-20$ \\
$\mathbf{4}$ & Glycerophospholipid metabolism & $1.172 \mathrm{E}-19$ & $9.055 \mathrm{E}-18$ \\
$\mathbf{6}$ & AMPK signaling pathway & $3.653 \mathrm{E}-18$ & $1.881 \mathrm{E}-16$ \\
$\mathbf{7}$ & Biosynthesis of unsaturated fatty acids & $1.133 \mathrm{E}-17$ & $5.002 \mathrm{E}-16$ \\
$\mathbf{1 0}$ & Phospholipase D signaling pathway & $2.606 \mathrm{E}-16$ & $8.053 \mathrm{E}-15$ \\
$\mathbf{1 3}$ & PPAR signaling pathway & $6.831 \mathrm{E}-16$ & $1.624 \mathrm{E}-14$ \\
$\mathbf{1 4}$ & Sphingolipid signaling pathway & $1.780 \mathrm{E}-15$ & $3.929 \mathrm{E}-14$ \\
$\mathbf{1 7}$ & Sphingolipid metabolism & $1.107 \mathrm{E}-14$ & $2.012 \mathrm{E}-13$ \\
$\mathbf{1 9}$ & Fatty acid elongation & $2.556 \mathrm{E}-13$ & $4.157 \mathrm{E}-12$ \\
$\mathbf{2 9}$ & Peroxisome & $1.579 \mathrm{E}-10$ & $1.683 \mathrm{E}-09$ \\
$\mathbf{3 1}$ & Cholesterol metabolism & $4.352 \mathrm{E}-10$ & $4.338 \mathrm{E}-09$ \\
$\mathbf{4 4}$ & Ether lipid metabolism & $2.027 \mathrm{E}-08$ & $1.423 \mathrm{E}-07$ \\
$\mathbf{4 5}$ & Fatty acid degradation & $2.497 \mathrm{E}-08$ & $1.715 \mathrm{E}-07$ \\
$\mathbf{4 8}$ & Glycerolipid metabolism & $8.699 \mathrm{E}-08$ & $5.600 \mathrm{E}-07$ \\
$\mathbf{9 0}$ & Steroid biosynthesis & $1.397 \mathrm{E}-05$ & $4.795 \mathrm{E}-05$ \\
$\mathbf{1 1 2}$ & ABC transporters & $3.483 \mathrm{E}-04$ & $9.608 \mathrm{E}-04$ \\
$\mathbf{1 1 3}$ & Fatty acid biosynthesis & $3.866 \mathrm{E}-04$ & $1.057 \mathrm{E}-03$ \\
$\mathbf{1 2 1}$ & Arachidonic acid metabolism & $1.075 \mathrm{E}-03$ & $2.744 \mathrm{E}-03$
\end{tabular}




\section{Figure legends}

Figure 1. ANGPTL3 knock-down efficiency in immortalized human hepatocytes. (A) ANGPTL3 mRNA expression of $\mathrm{IHH}$ cells treated with non-targeting shRNA (control) and shRNA targeting ANGPTL3 (shANGPTL3). The values represent mean $\pm S D, n=3$. (B) ANGPTL3 protein levels in control and ANGPTL3 knock-down cells (data represent mean fold change $\pm S D, n=3$ ). (C) Representative western blot image of ANGPTL3 expression in control and knock-down cells with corresponding total protein. ${ }^{* *} p<0.001,{ }^{*} p<0.05$.

Figure 2. MUFAs are decreased and PUFAs increased in ANGPTL3 knock-down cells. (A) Sums of saturated and unsaturated fatty acids determined by gas chromatography. The values represent mean $\pm S D, n=6 .{ }^{* *} p<0.01,{ }^{* * *} p<0.001$. (B) PCA of individual FA species. The samples located furthest from the origin of the PCA biplot (marked as + ) on one side contain relatively more of the lipid species furthest on that same side. The longer the distance between two samples on the plot the more their lipid profiles differ from each other. The percentages represent the proportion of the variation in the data each principal component axis explains. Ctrl=control cells treated with nontargeting RNA, shANGPTL3/ShA3=cells treated with shRNA against ANGTPL3.

Figure 3. Knocking down ANGPTL3 enhances the production of lipid mediators. (A) PLS-DA analysis of lipid mediator concentrations. The score plot shows clear separation between the control (red dots) and knock-down (shANGPTL3; green dots) samples. Coloured spherical areas display the $95 \%$ confidence region. (B) VIP scores of lipid mediator variables that contribute most to the separation in the PLS-DA model. All of these mediators are more abundant in the ANGPTL3 knock-down (shA3) cells as indicated by the colour scale. RvD6=resolvin D6, $\mathrm{PGD}_{2}=$ prostaglandin $D_{2}, T_{x} B_{2}=$ thromboxane $B_{2}, H D P A=$ hydroxydocosapentaenoic acid, MaR=maresin, $L X A_{4}=$ lipoxin $A_{4}$, $\mathrm{LTB}_{4}=$ leukotriene $\mathrm{B}_{4}, \mathrm{HDHA}=$ hydroxydocosahexaenoic acid, HETE=hydroxyeicosatetraenoic acid.

Figure 4. Cholesterol ester synthesis and SOAT1 expression are reduced in ANGPTL3 cells. (A) Total cholesterol esters (CE) in control and ANGPTL3 knock-down cells (data represent mean \pm SD, $n=6$ ). (B) SOAT1 mRNA expression in control and ANGPTL3 knock-down cells (mean \pm SD, 
$\mathrm{n}=6)(\mathrm{C})$ representative Western blot of SOAT1 in control and ANGPTL3 knock-down cells (D) Quantification of protein expression of SOAT1 in ANGPTL3 knock-down and control cells (mean \pm $\mathrm{SD}, \mathrm{n}=4)$. (E) $\left[{ }^{3} \mathrm{H}\right]$ acetic acid labeled CE in control and ANGPTL3 knock-down cells (mean $\pm \mathrm{SD}$, $\mathrm{n}=12$ ). (F) $\left[{ }^{3} \mathrm{H}\right.$ ]oleic acid incorporation into CE in control and ANGPTL3 knock-down cells (mean \pm $\mathrm{SD}, \mathrm{n}=18) .{ }^{* *} \mathrm{p}<0.001,{ }^{* *} \mathrm{p}<0.001$.

Figure 5. ANGPTL3 LOF carriers have altered lipoprotein fatty acid profiles. PCA of the lipoprotein fatty acid profiles of control and ANGPTL3 LOF subjects. Lipoprotein fractions were isolated from the plasma of ANGPTL3 LOF homozygote and control subjects by sequential density ultracentrifugation and fatty acids were analyzed by gas chromatography. Sample markings: Group+Fraction; $\mathrm{C}=$ control, $\quad \mathrm{HZ}=\mathrm{ANGPTL3}$ LOF homozygote, $\mathrm{V}=\mathrm{VLDL}, \quad \mathrm{L}=\mathrm{LDL}, \quad \mathrm{H}=\mathrm{HDL}$. $\mathrm{DMA}=$ dimethylacetal.

Figure 6. PUFAs accumulate in TAGs of lipoproteins derived from ANGPTL3 LOF homozygotes. TAG of lipoproteins from ANGPTL3 loss-of-function and control subjects was analyzed by ESIMS/MS. Bar graphs are shown for VLDL (upper panel) and LDL (lower panel) fractions, but also in HDL the effect was clear and very similar to that seen in LDL (Supplementary table 12). The values represent mean $\pm S D, n=4-10 .{ }^{*} p<0.05,{ }^{* *} p<0.01,{ }^{* * *} p<0.001$.

Figure 7. ANGPTL3 deficiency alters the surface lipids of lipoprotein particles. (A) SM/PC ratio of different lipoprotein fractions. ANGPTL3=ANGPTL3 LOF homozygote. (B) PCA of the relative profile of SM in LDL. C=control, HZ=ANGPTL3 LOF homozygote. All species have a sphingosine 18:1 backbone, except SM 24:2 is likely a mixture of 18:1/24:2 and 18:2/24:1. (C) LysoPC species profile of LDL derived from control subjects and ANGPTL3 LOF homozygotes. The values represent mean $\pm S D, n=5-10 .{ }^{*} p<0.05,{ }^{* *} p<0.01,{ }^{* * *} p<0.001$. 
Figure 1

A

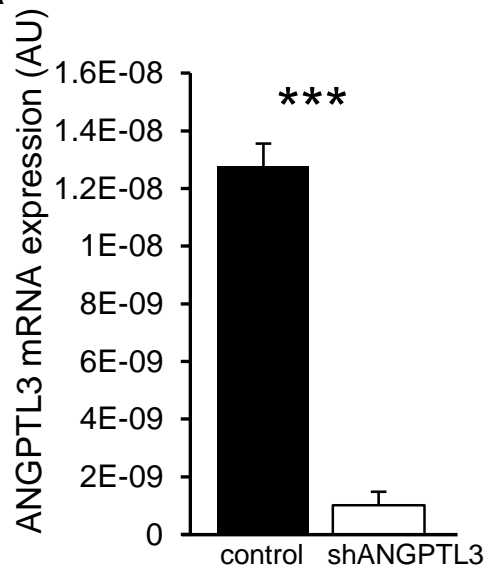

B

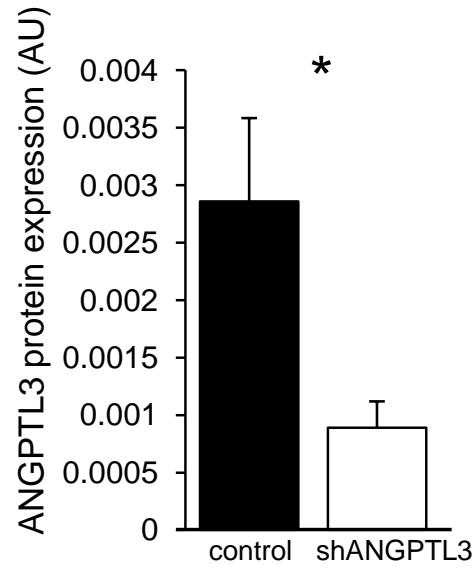

C

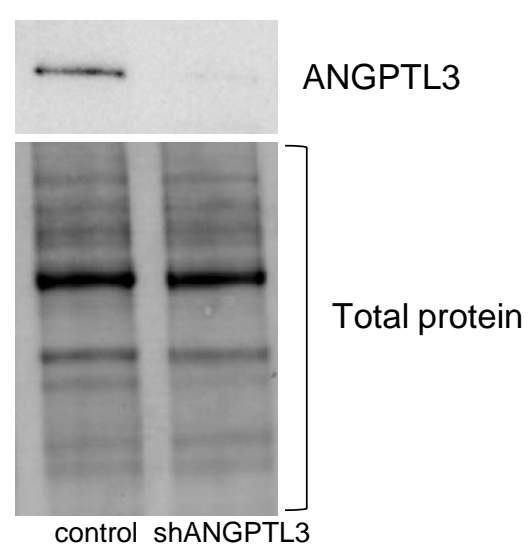


Figure, 2

\section{A}

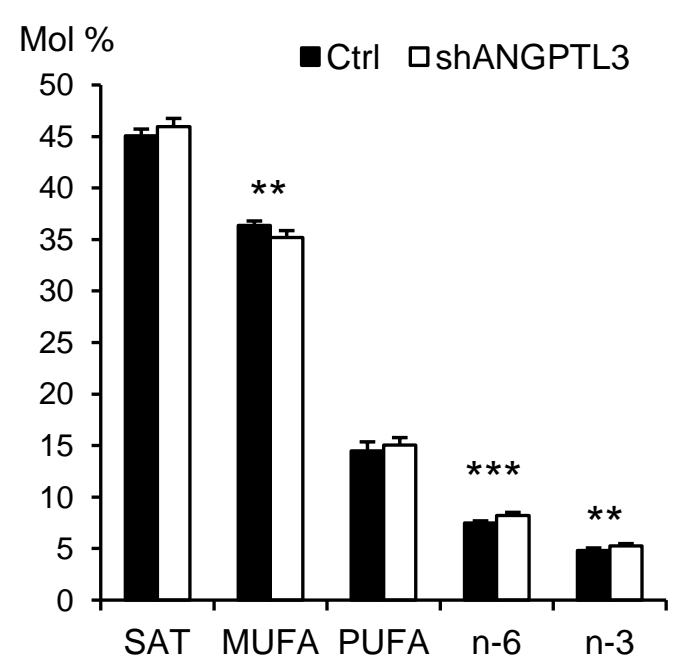

B

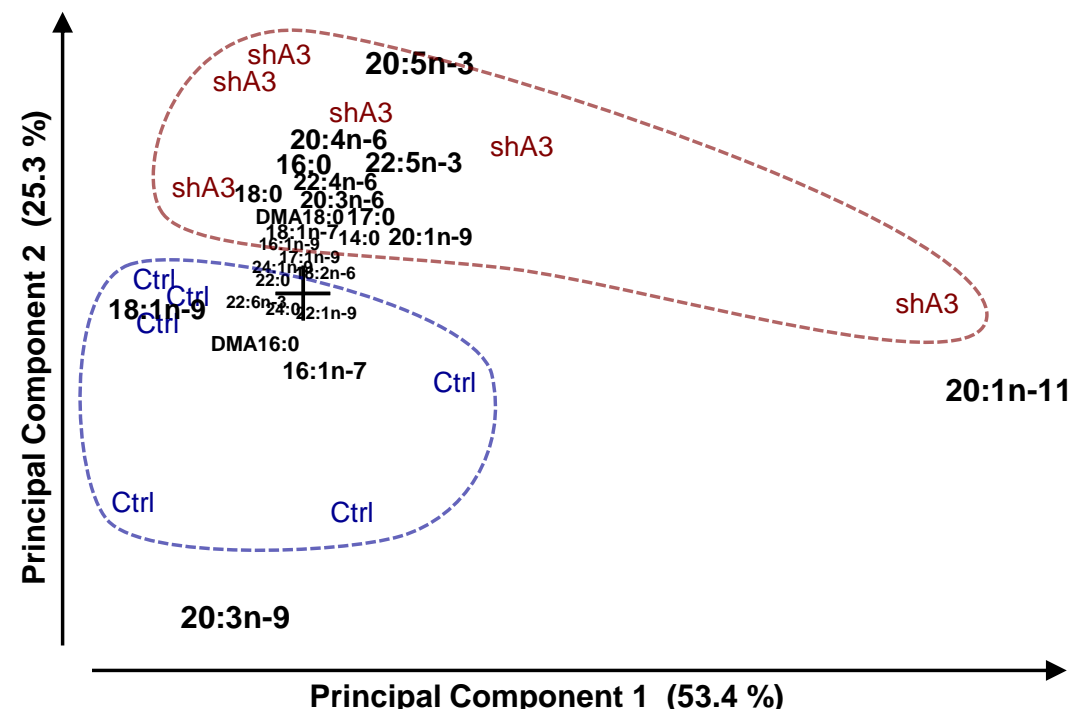

Principal Component 1 (53.4\%) 
A

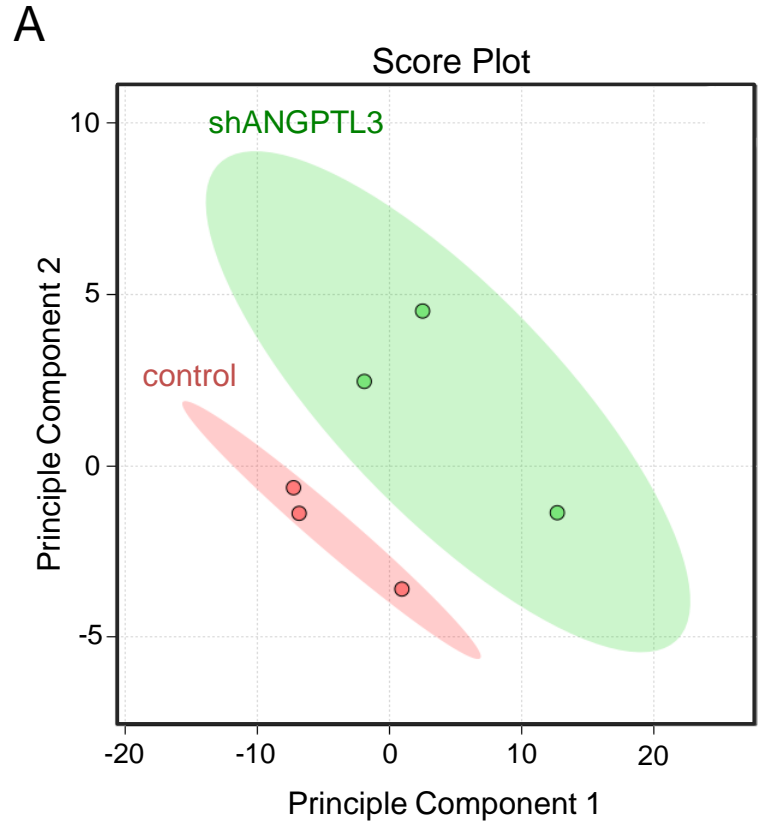

Figures.
B

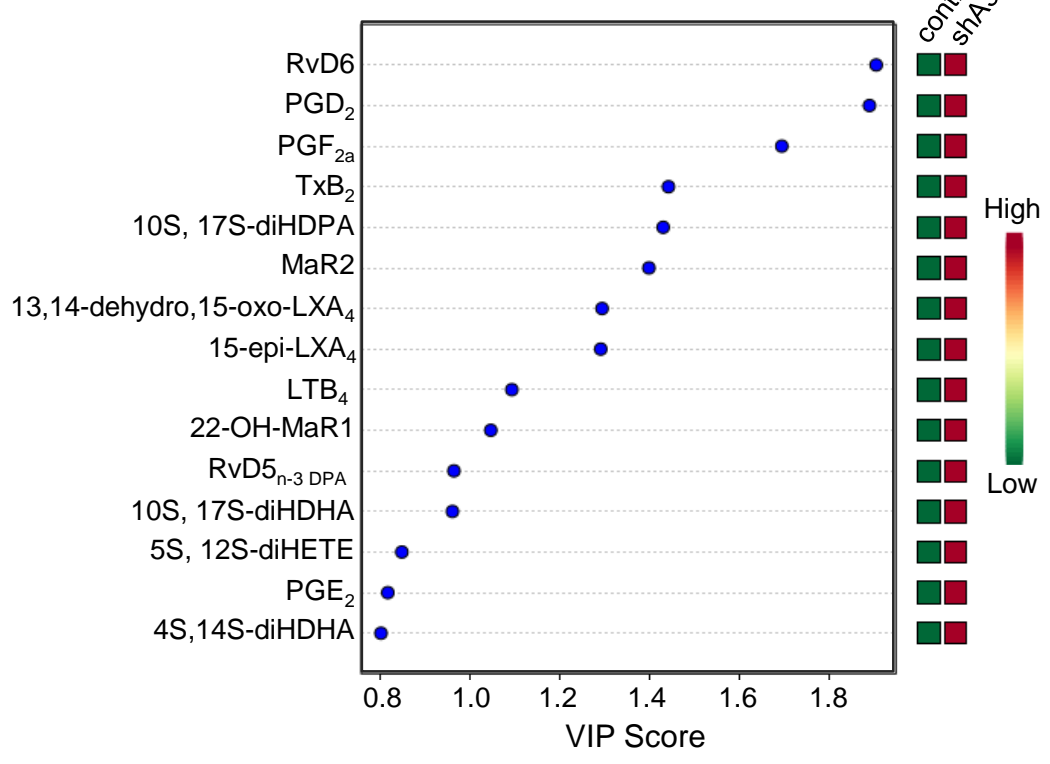


Figure 4

A

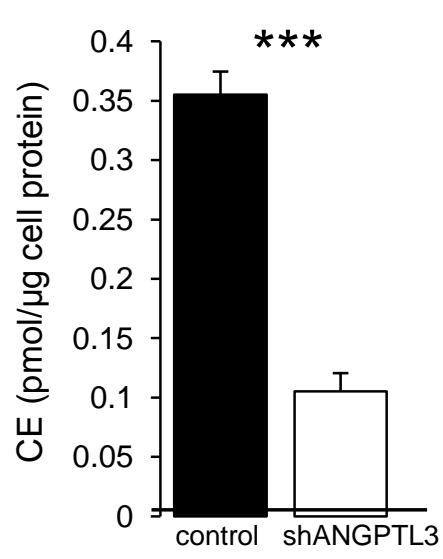

D

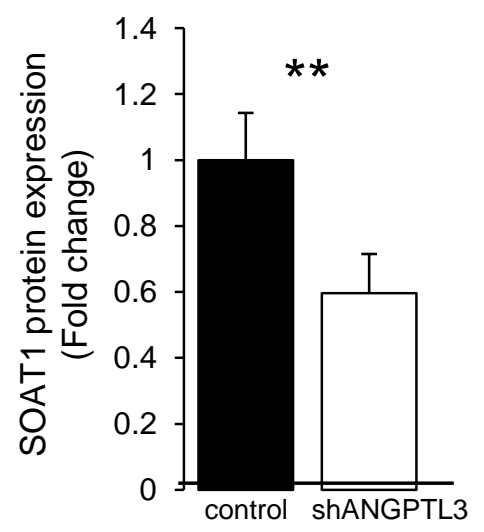

B

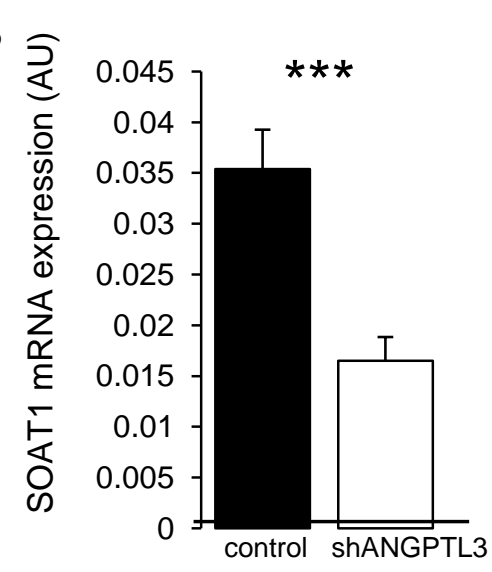

E

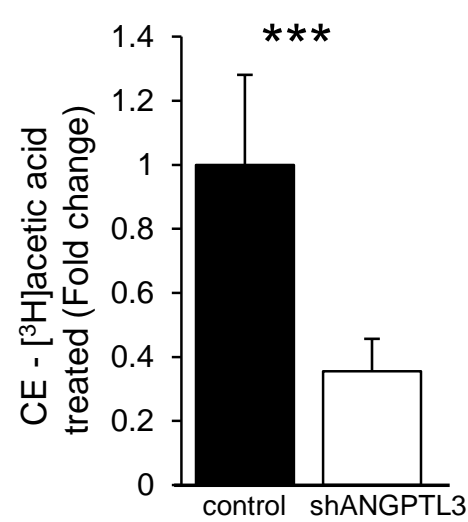

C SOAT1

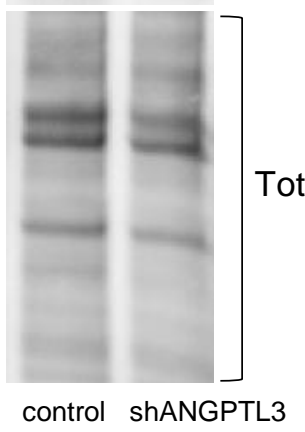

F

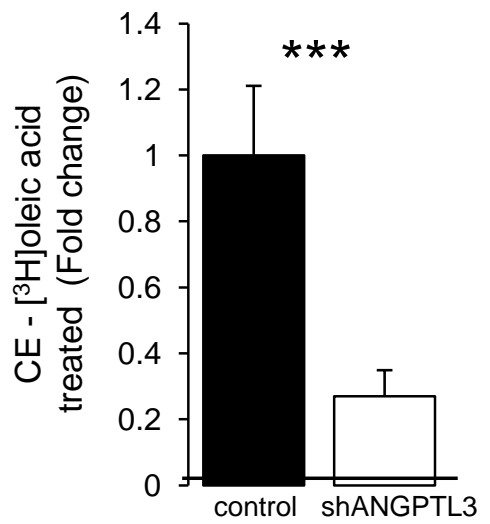


Figure 5

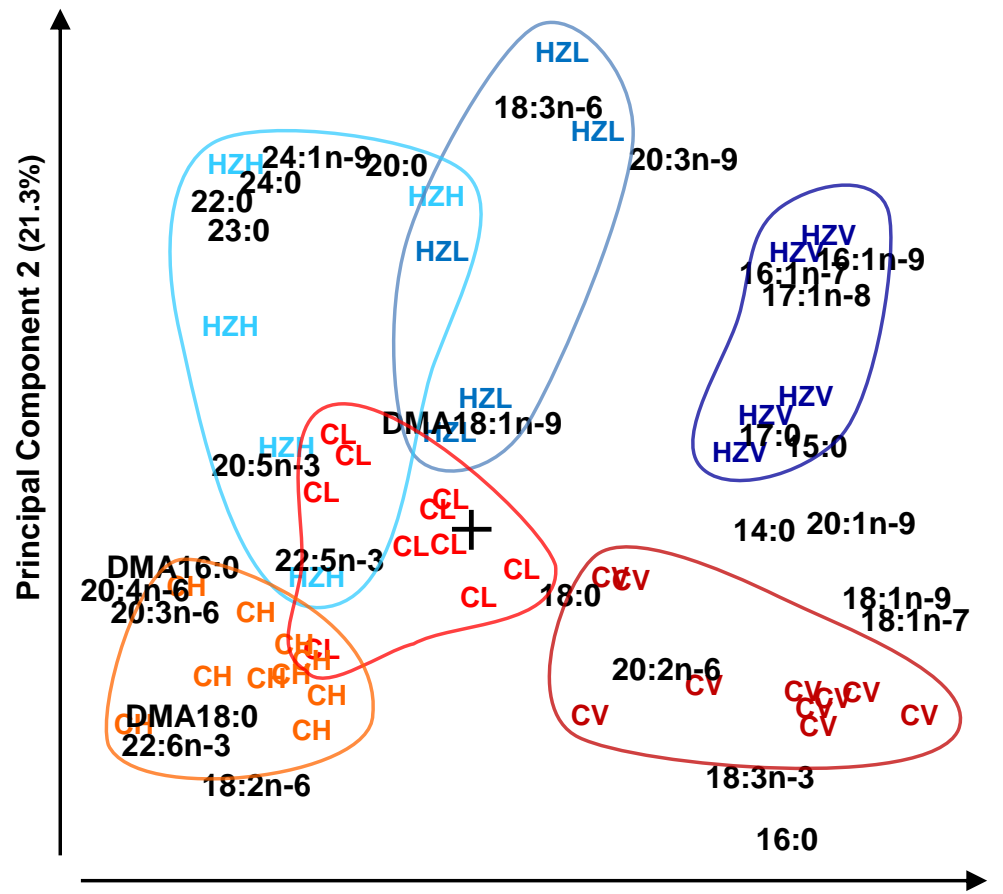

Principal Component 1 (31.7\%) 
Eigure 6
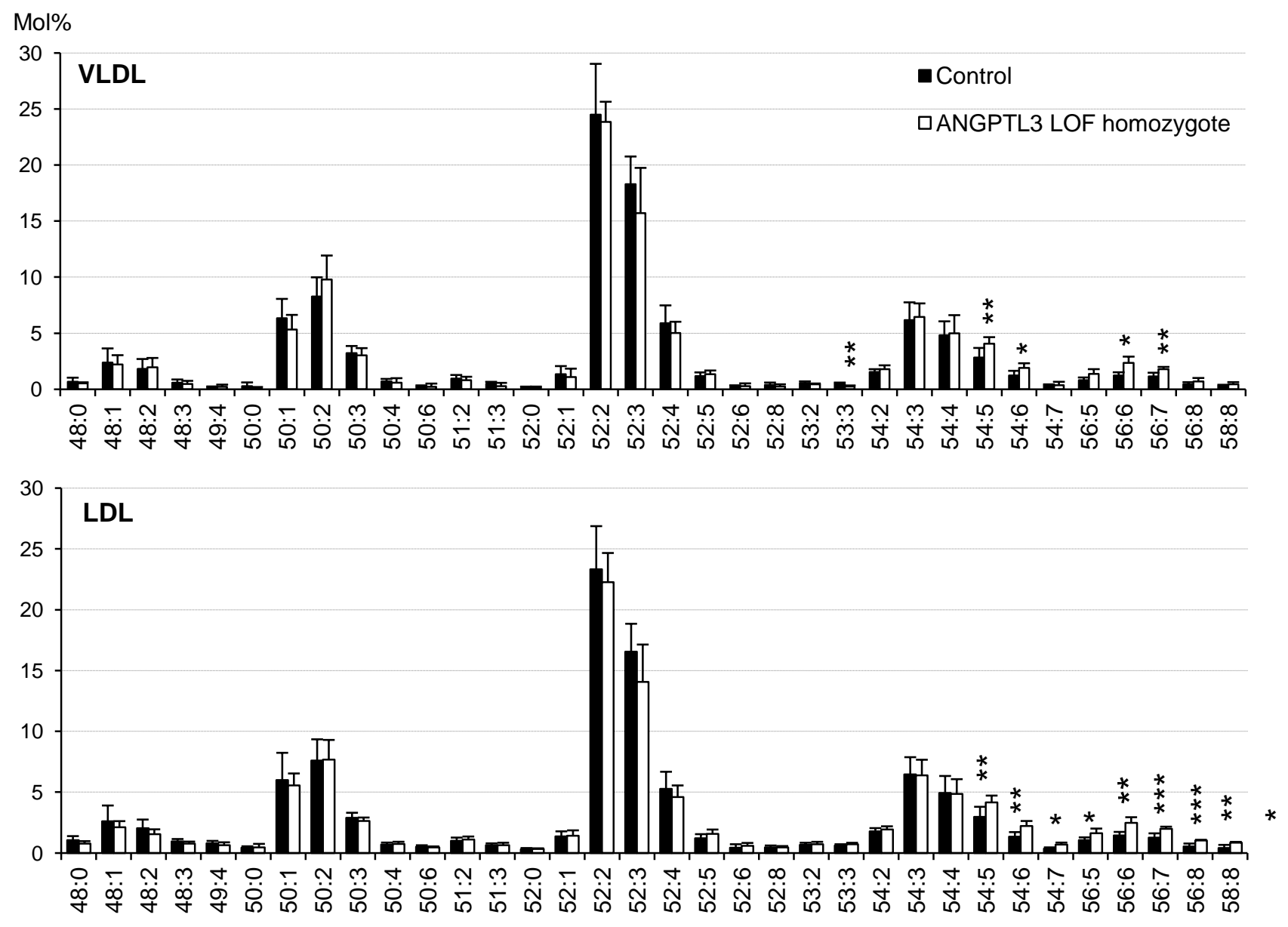
Figure-7

A

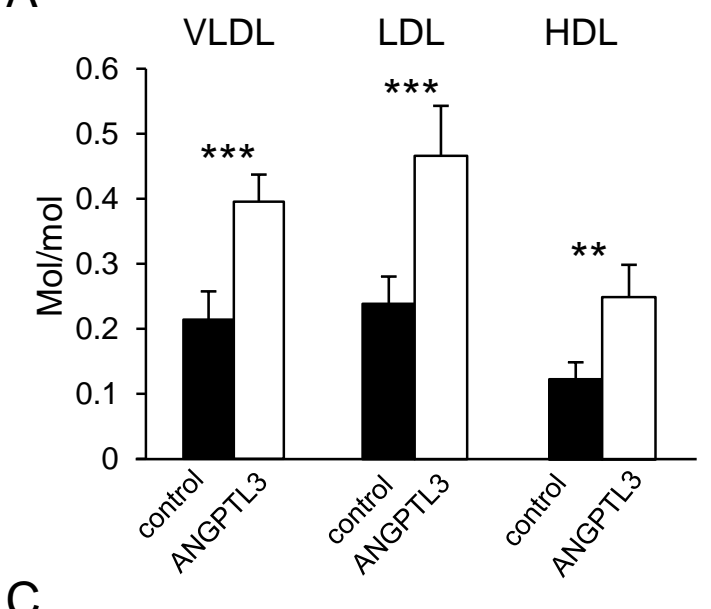

B

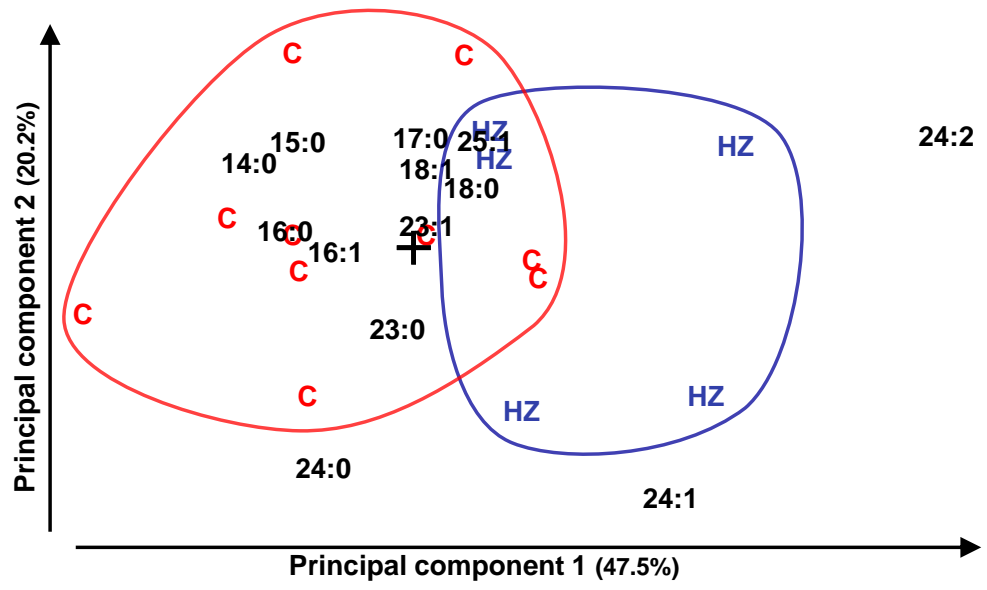

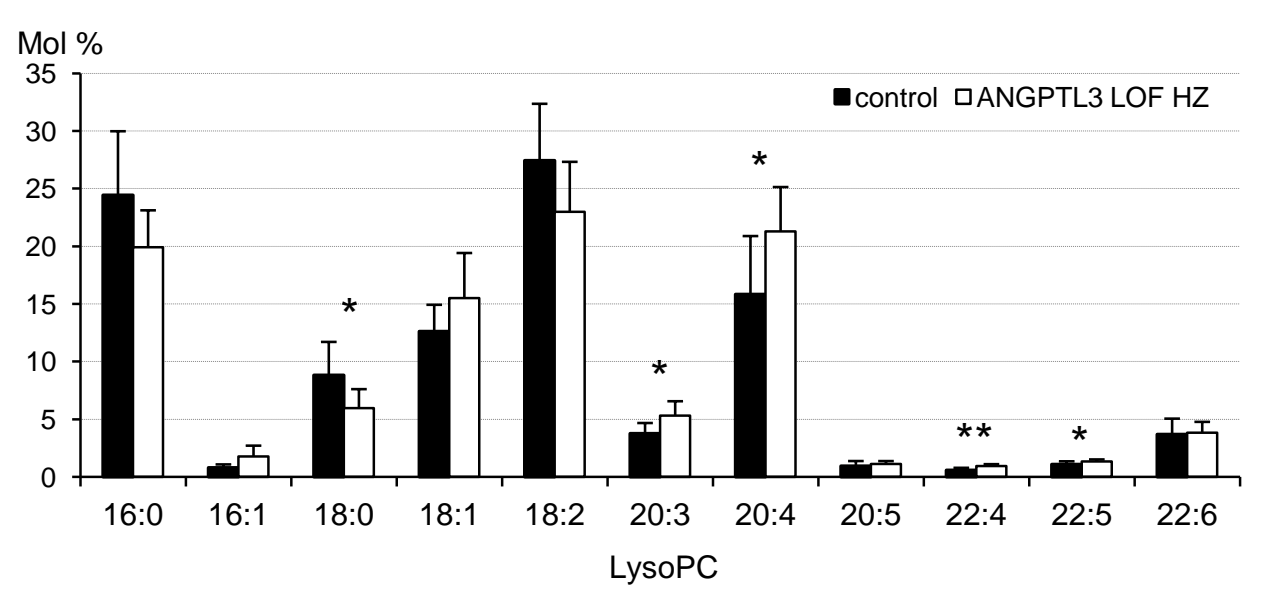




\section{Supplementary material to:}

ANGPTL3 deficiency alters the lipid profile and metabolism of cultured hepatocytes and human lipoproteins. Hanna Ruhanen, Nidhina Haridas P.A., Ilenia Minicocci, Juuso H. Taskinen, Francesco Palmas, Alessia di Costanzo, Laura D’Erasmo, Jari Metso, Jennimari Partanen, Jesmond Dalli, You Zhou, Marcello Arca, Matti Jauhiainen, Reijo Käkelä \& Vesa M. Olkkonen* Biochimica et Biophysica Acta - Molecular and Cell Biology of Lipids. *Corresponding author at: Minerva Foundation Institute for Medical Research, Biomedicum 2U, Tukholmankatu 8, FI-00290 Helsinki, Finland; Tel +358-2-94125705, e-mail vesa.olkkonen@helsinki.fi

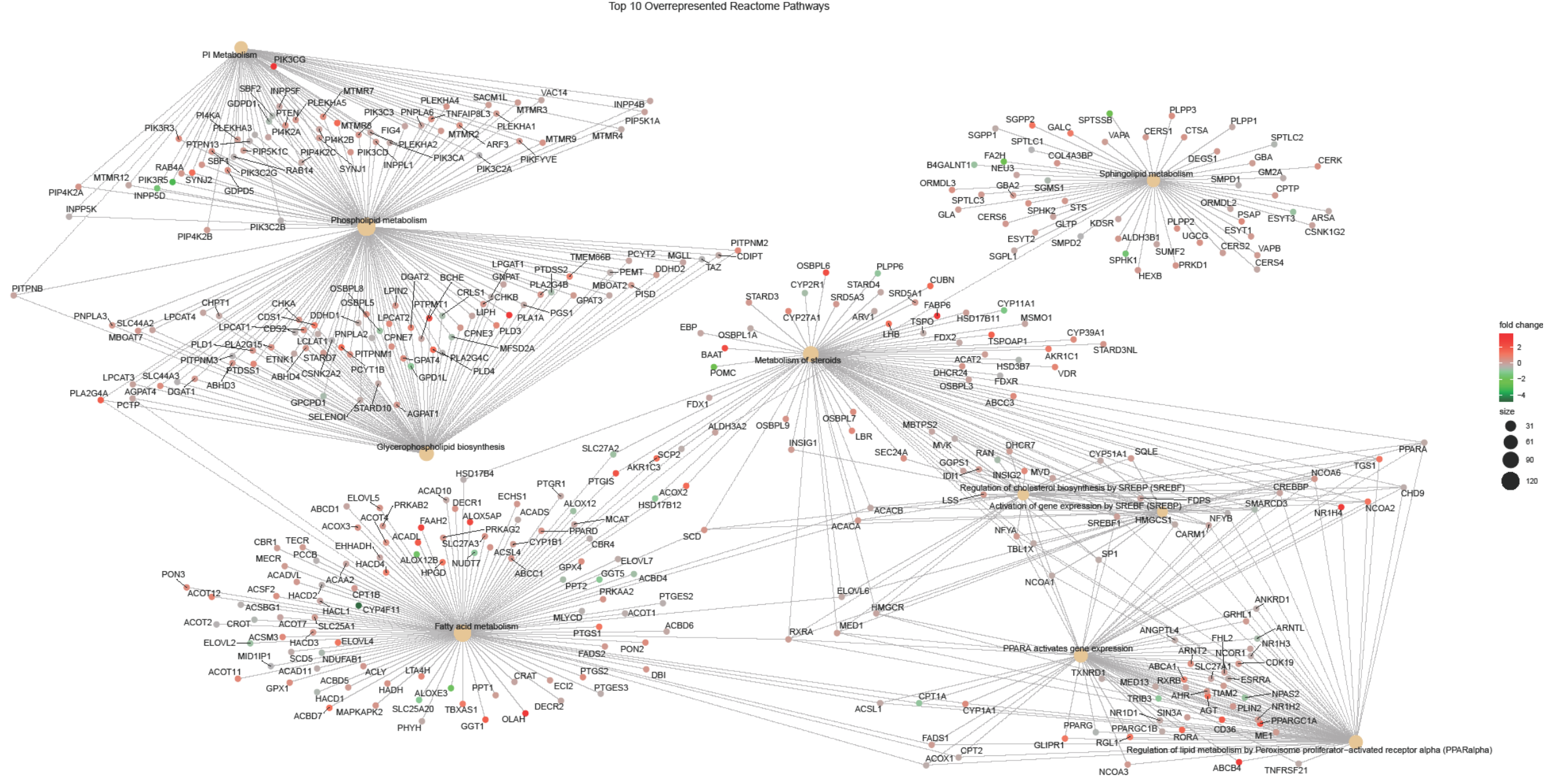

Supplementary Figure 1. Ten most altered pathways of Reactome pathway analysis in ANGPTL3 knock-down cells. Only statistically significantly up/downregulated genes were used. Red dots mark upregulated genes and green dots downregulated genes. The brighter the color the bigger the fold change. 
A

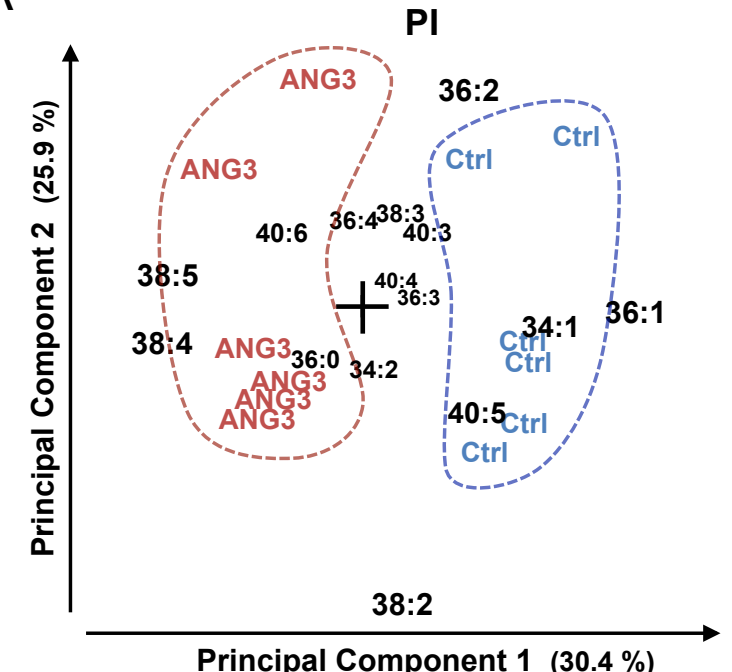

C

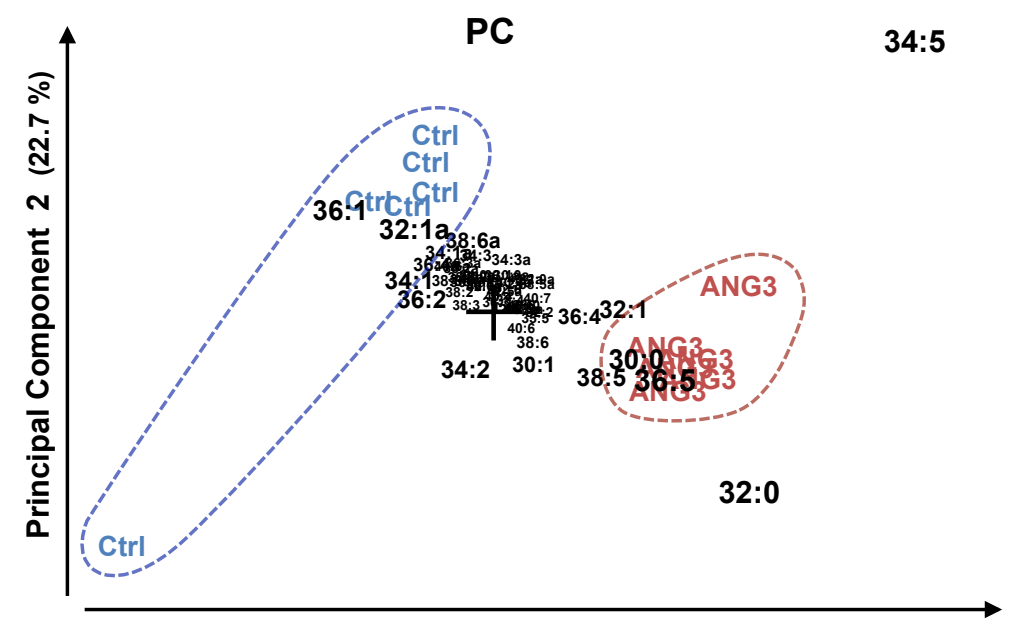

Principal Component 1 (52.6\%)
B $\quad P E$

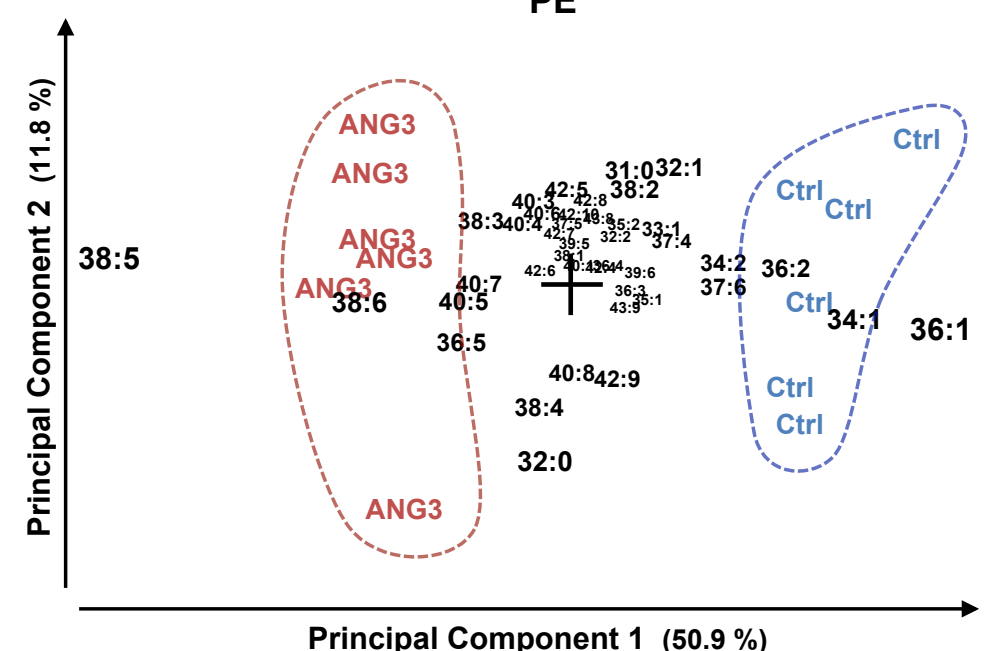

Principal Component 1 (50.9\%)

Supplementary Figure 2. Principal component analysis of the phospholipids of ANGPTL3 knock-down and control IHH cells. The samples located furthest from the origin of the PCA biplot (marked as + ) on one side contain relatively more of the lipid species furthest on that same side. The longer the distance between two samples on the plot the more their lipid profiles differ from each other. Arrows show the direction of the Principal Components 1 and 2 (PC1 and PC2) and the percentages represent the proportion of the variation in the data each PC axis explains. (A) PCA of phosphatidylinositol (PI) species. The species are identified as 'number of carbons':'number of double bonds'. Ctrl=control ANG3=ANGPTL3 knock-down cells. (B) PCA of phosphatidylethanolamines (PE). (C) PCA of phosphatidylcholines (PC). a=alkyl-acyl species. 


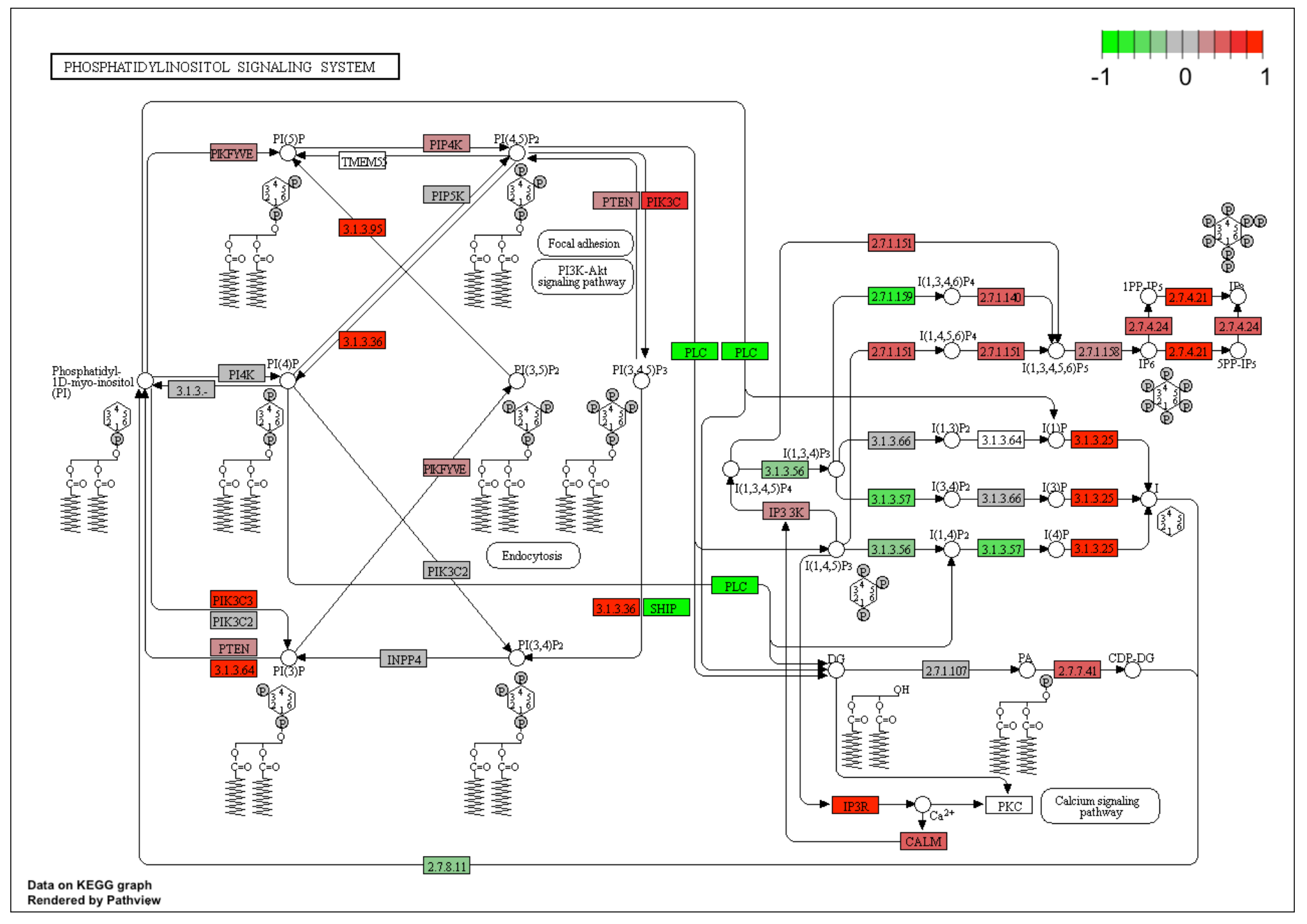

Supplementary Figure 3. KEGG phosphatitylinositol signaling pathway of ANGPTL3 knock-down cells. Red boxes mark upregulated genes and green boxes downregulated genes. The brighter the color the bigger the fold change. 


\section{Supplementary material to:}

ANGPTL3 deficiency alters the lipid profile and metabolism of cultured hepatocytes and human lipoproteins. Hanna Ruhanen, Nidhina Haridas P.A., Ilenia Minicocci, Juuso H. Taskinen, Francesco Palmas, Alessia di Costanzo, Laura D’Erasmo, Jari Metso, Jennimari Partanen, Jesmond Dalli, You Zhou, Marcello Arca, Matti Jauhiainen, Reijo Käkelä \& Vesa M. Olkkonen*. Biochimica et Biophysica Acta - Molecular and Cell Biology of Lipids. *Corresponding author at: Minerva Foundation Institute for Medical Research, Biomedicum 2U, Tukholmankatu 8, FI-00290 Helsinki, Finland; Tel +358-2-94125705, e-mail vesa.olkkonen@helsinki.fi

Supplementary table 1. Sequences of the GPCR primers used.

\begin{tabular}{lll} 
mRNA & \multicolumn{2}{c}{ Sequence 5' to 3' } \\
\hline ANGPTL3 & $\begin{array}{l}\text { Forward } \\
\text { Reverse }\end{array}$ & $\begin{array}{l}\text { CCAGAACACCCAGAAGTAACT } \\
\text { TCTGGGTTCTTGAATACTAGTC }\end{array}$ \\
SOAT1 & $\begin{array}{l}\text { Forward } \\
\text { Reverse }\end{array}$ & CAAGGCGCTCTCTCTTAGATG \\
& GGAAACAACGGTAGGAAA \\
$\beta$-actin & Forward & GACAGGATGCAGAAGGAGATT \\
& Reverse & TGATCCACATCTGCTGGAAGG \\
RPLP0 & $\begin{array}{ll}\text { Forward } \\
\text { Reverse }\end{array}$ & TGGTCATCCAGCAGGTGTTCGA \\
& ACACAGGCAACATTGCG
\end{tabular}

Supplementary table 2. Characteristics of the study participants.

\begin{tabular}{lcc} 
& Controls & ANGPTL3 LOF homozygotes \\
\hline $\mathrm{n}$ & 10 & 5 \\
Age (years) & $55.3( \pm 15.6)$ & $59.2( \pm 22.5)$ \\
Sex $(\%$ females $)$ & 60 & 60 \\
BMl $\left(\mathrm{kg} / \mathrm{m}^{2}\right)$ & $27.9( \pm 3.9)$ & $28.3( \pm 5.2)$ \\
\hline
\end{tabular}

BMI: body mass index; \pm standard deviation (SD) 


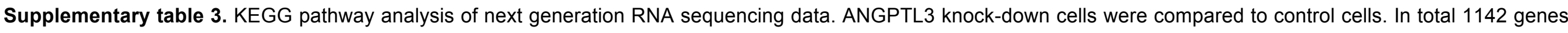
were used in the analysis.

KEGG ID Description

hsa00562 Inositol phosphate metabolism

hsa04070 Phosphatidylinositol signaling system

hsa01212 Fatty acid metabolism

hsa00564 Glycerophospholipid metabolism

hsa05205 Proteoglycans in cancer

8.94E-19 5.52E-17

pvalue

p.adjust

qvalue genelD

2.33E-25 7.20E-23 3.16E-23

$1.58 \mathrm{E}-23 \quad 2.44 \mathrm{E}-21$

1.20E-22 1.23E-20

17E-19 9.05E-18

$52 \mathrm{E}-17$

PIK3CG/MTMR8/SYNJ2/IMPAD1/PLCH2/IPMK/MTMR7/IMPA1/PLCE1/PLCG1/ISYNA1/PLCH1/PTEN/PLCD4/IMPA2/ PIK3C2G/PIK3CD/PIP4K2A/PIK3C3/PIKFYVE/MTMR2/SYNJ1/INPP5A/INPPL1/MTMR3/PIP4K2B/PIP5K1C/PI4KA/SA CM1L/PIP5K1A/PI4K2B/INPP5F/PI4K2A/FIG4/INPP4B/ALDH6A1/PLCB3/PLCD3/PIP4K2C/PIK3CA/CDIPT/MTMR4/IN PP5K/PLCG2/INPP1/PIK3C2B/PIK3C2A/PLCD1/PLCB4/INPP5D

1.07E-21 ITPR2/MTMR8/SYNJ2/CDS1/IP6K3/IMPAD1/PIK3R3/IPMK/MTMR7/IMPA1/PLCE1/PLCG1/CALM3/DGKI/PTEN/PLCD 4/IMPA2/PIK3C2G/PIK3CD/PIP4K2A/PIK3C3/IP6K1/IP6K2/PIKFYVE/MTMR2/SYNJ1/INPP5A/INPPL1/MTMR3/PIP4K 2B/PIP5K1C/PI4KA/DGKD/SACM1L/PIP5K1A/PI4K2B/INPP5F/PI4K2A/INPP4B/PLCB3/PLCD3/PIP4K2C/PIK3CA/CDI PT/MTMR4/DGKG/DGKA/INPP5K/PLCG2/ITPR1/CDS2/INPP1/PIK3C2B/PIK3C2A/PLCD1/PLCB4/INPP5D

5.42E-21 ACADL/CPT1C/ELOVL4/HACD4/HADH/ACOX3/ELOVL5/SCD/ACSL4/HACD3/ACACA/ECHS1/CPT1B/FADS2/ACADV L/TECR/MECR/FADS1/PPT1/ACAT2/MCAT/ACAA2/EHHADH/ACSL1/ELOVL6/ACADS/CPT2/ELOVL7/SCD5/ACOX1/ SCP2/CBR4/HACD2/HSD17B4/ACSBG1/HACD1/ACADSB/PPT2/ELOVL2/CPT1A/HSD17B12

3.98E-18 PLA2G4A/PLPP4/PLA2G4C/PCYT1B/CDS1/LYPLA1/PLD3/PTDSS1/LPCAT2/LCAT/GPAT4/AGPAT4/LPCAT1/PLPP3/ PLPP2/DGKI/PLA2G15/LYPLA2/PLD1/PLA2G4B/CHKB/LPIN2/CHKA/MBOAT7/AGPAT1/GPAT3/PISD/DGKD/LPCAT 3/PNPLA6/PCYT2/GNPAT/LPCAT4/MBOAT2/ETNK1/PLPP1/CHPT1/PGS1/CDIPT/LCLAT1/SELENOI/DGKG/DGKA/P EMT/LPGAT1/CDS2/PLD4/TAZ/GPCPD1/CRLS1/PTDSS2/GPD1L

HCLS1/TWIST1/SHH/KDR/DCN/FASLG/ITGB3/ITPR2/WNT10B/IGF2/WNT5A/ERBB4/TLR2/TLR4/ITGAV/SDC2/FZD5 /PIK3R3/HSPG2/TGFB2/PLCE1/PLCG1/SDC4/ITGB1/AKT2/THBS1/CASP3/FZD4/PIK3CD/HIF1A/FZD7/TGFB1/IGF1 R/EGFR/PAK1/STAT3/IQGAP1/GAB1/MTOR/MSN/PRKACB/PDPK1/PTK2/CTNNB1/SOS1/MAPK1/CAMK2G/RAC1/R HOA/ERBB2/RPS6KB1/ROCK2/MDM2/PTPN11/KRAS/MAPK3/SDC1/DDX5/FGFR1/CCND1/AKT1/CAV1/PIK3CA/CB L/GPC1/SMO/WNT7B/CAV2/PLCG2/ELK1/ITPR1/EZR/TFAP4/FGF2/ITGA2/PTCH1/WNT3/WNT9A/ERBB3/TIAM1

hsa04152 AMPK signaling pathway

$3.65 \mathrm{E}-18 \quad 1.88 \mathrm{E}-16$

8.27E-17 ARGC1A/CD36/CPT1C/LIPE/PPP2R1A/PIK3R3/TSC1/LEPR/IRS1/AKT2/PIK3CD/SCD/PRKAA2/IGF1R/PRKAG2/A DIPOR1/ACACA/PRKAB2/CPT1B/PFKL/TSC2/EIF4EBP1/MTOR/PPP2R5B/PDPK1/FOXO3/INSR/CCNA2/RAB10/SR EBF1/RPS6KB1/PRKAA1/RAB8A/PRKAB1/EEF2K/PPP2R5C/HMGCR/PPP2R5A/EEF2/CCND1/SIRT1/AKT1/PIK3CA/ ACACB/PFKFB2/ADIPOR2/SCD5/MLYCD/PPP2CA/RAB14/IRS2/PPARG/FOXO1/SLC2A4/PCK2/CPT1A/HNF4A

hsa01040 Biosynthesis of unsaturated fatty acids

hsa04931 Insulin resistance

$1.13 \mathrm{E}-17 \quad 5.00 \mathrm{E}-16$ ACOX1/SCP2/ACOT1/ACOT4/ACOX3/ELOVL5/SCD/HACD3/FADS2/TECR/FAD

5.37E-17 2.07E-15 9.11E-16

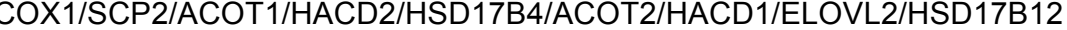

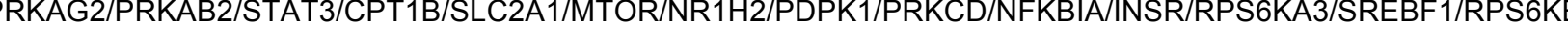
1/PTPN11/PRKAA1/OGT/PRKAB1/SLC27A1/PRKCZ/AKT1/PIK3CA/GSK3B/ACACB/TNFRSF1A/SLC27A4/PPARGC1 B/PPARA/NR1H3/NFKB1/IRS2/FOXO1/SLC2A4/SLC27A2/PCK2/CPT1A/TRIB3

hsa04933 AGE-RAGE signaling pathway in diabetic complications

hsa04072 Phospholipase D signaling pathway

$6.03 \mathrm{E}-15$ COL1A1/CXCL8/AGT/JUN/IL6/MAPK8/CDKN1B/PIK3R3/EDN1/TGFB2/PLCE1/PLCG1/BAX/TGFBR1/AKT2/CASP3/P LCD4/PRKCE/PIK3CD/TGFB1/SERPINE1/STAT3/PRKCD/CDK4/MAPK1/STAT1/RAC1/KRAS/MAPK3/PIM1/PLCB3/C CND1/TGFBR2/PLCD3/PRKCZ/AKT1/ICAM1/PIK3CA/STAT5B/BCL2/JAK2/PLCG2/NFKB1/PLCD1/FOXO1/CCL2/THB $\mathrm{D} / \mathrm{PLCB} 4 / \mathrm{F} 3$

2.61E-16 8.05E-15 3.54E-15 F2/PIK3CG/LPAR4/LPAR3/PDGFB/PLA2G4A/CXCL8/AGT/PLA2G4C/PDGFC/PTGFR/PDGFRA/CYTH2/LPAR2/RAP GEF4/F2R/PIK3R3/AGPAT4/TSC1/PLPP3/ADCY6/PLCG1/AKT2/PDGFRB/PLPP2/DGKI/SPHK2/PIK3CD/PLD1/PLA2 G4B/EGFR/AGPAT1/TSC2/GAB1/MTOR/GNAS/INSR/PIP5K1C/SOS1/MAPK1/DGKD/RHOA/PIP5K1A/PTPN11/KRAS /MAPK3/SHC1/PLCB3/AKT1/CYTH3/PIK3CA/PLPP1/ADCY3/DGKG/DGKA/PLCG2/PTK2B/PDGFA/LPAR1/PLCB4/SP HK1/PIK3R5

hsa04920 Adipocytokine signaling pathway

4.18E-16 1.18E-14

5.17E-15

PPARGC1A/CD36/CPT1C/PRKCQ/MAPK8/LEPR/IRS1/AKT2/PRKAA2/ACSL4/PRKAG2/ADIPOR1/PRKAB2/STAT3/C PT1B/SLC2A1/MTOR/NFKBIA/RXRA/RXRB/PTPN11/PRKAA1/PRKAB1/NFKBIB/AKT1/ACSL1/ACACB/TNFRSF1A/P PARA/ADIPOR2/JAK2/ACSBG1/NFKB1/IRS2/SLC2A4/PCK2/CPT1A/POMC/TNFRSF1B

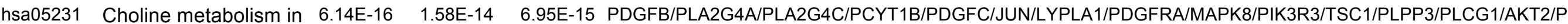
cancer
DGFRB/PLPP2/DGKI/PIK3CD/HIF1A/PLD1/PLA2G4B/CHKB/CHKA/EGFR/TSC2/EIF4EBP1/MTOR/PDPK1/PIP5K1C/ 
hsa03320 PPAR signaling pathway

hsa04071 Sphingolipid signaling pathway

hsa05165 Human

hsa04919 Thyroid hormone signaling pathway

8.69E-15 1.68E-13

Sphingolipid metabolism

hsa00600

hsa01521

EGFR tyrosine kinase

hsa00062 Fatty acid elongation

hsa04144 Endocytosis

$2.56 \mathrm{E}-13 \quad 4.16 \mathrm{E}-12$

1.58E-12 2.44E-11

17E-12 7.27E-11

FoxO signaling pathway

hsa04140 Autophagy - animal

5.17E-12 7.27E-11

hsa04068 inhibitor resistance

SOS1/MAPK1/DGKD/RAC1/PIP5K1A/RPS6KB1/SLC44A2/KRAS/MAPK3/AKT1/PIK3CA/PLPP1/CHPT1/SP1/DGKG/D GKA/SLC44A3/GPCPD1/PDGFA

5 FABP6/CD36/ACADL/FABP3/AQP7/ACOX2/CPT1C/FABP5/CYP27A1/PLTP/ACOX3/PPARD/SCD/ACSL4/DBI/PLIN2/ CPT1B/FADS2/PDPK1/ME1/RXRA/RXRB/EHHADH/SLC27A1/ACSL1/SLC27A4/CPT2/PPARA/ANGPTL4/HMGCS1/S CD5/NR1H3/ACOX1/SCP2/ACSBG1/PPARG/GK/SLC27A2/OLR1/PCK2/CPT1A

4 SGPP2/ADORA1/S1PR1/MAPK8/PPP2R1A/PIK3R3/BAX/AKT2/GNAI2/PTEN/SPHK2/S1PR2/PRKCE/PIK3CD/PLD1/A BCC1/NSMAF/CERS6/DEGS1/SPTLC3/CERS1/PPP2R5B/PDPK1/GNAI1/MAPK1/RAC1/CERS2/RHOA/ROCK2/KRA S/PPP2R5C/MAPK3/PPP2R5A/PLCB3/PRKCZ/AKT1/PIK3CA/SGPL1/CERS4/TNFRSF1A/CTSD/BCL2/SGPP1/PPP2 CA/SMPD1/NFKB1/SPTLC1/SMPD2/SPTLC2/SGMS1/S1PR4/PLCB4/SPHK1

5.16E-14 SPP1/ATP6V0D2/FASLG/ITGB3/COL1A1/LAMA1/TNC/WNT10B/WNT5A/ATP6V1H/PTGER4/ITGAV/ITGB8/TCIRG1/ CDKN1B/FZD5/CCNE1/PPP2R1A/PTGS2/PIK3R3/ATP6V1C1/TSC1/BAX/ITGB1/RB1/AKT2/PDGFRB/THBS1/CASP3/ PTEN/ATM/FZD4/PIK3CD/FZD7/HDAC2/ATP6V0B/EGFR/TCF7L2/IRF3/TSC2/E2F1/EIF4EBP1/MTOR/IFNAR1/RBL1/ PPP2R5B/PRKACB/GNAS/ATP6V1G1/APC/CDK4/ATP6V1B2/PTK2/ATP6AP1/ATP6V0A1/CTNNB1/SOS1/ATP6V1E1 /MAPK1/ATP6V1F/STAT1/CCNA2/BCAP31/PKM/RPS6KB1/CCND3/MDM2/UBE3A/CREBBP/KRAS/PPP2R5C/NOTC H1/MAPK3/TADA3/PPP2R5A/BAD/TICAM1/PRKCI/CCND1/CASP8/PRKCZ/AKT1/ATP6V0D1/PIK3CA/ATP6V1C2/GS K3B/TNFRSF1A/ATP6V0A2/WNT7B/ATP6V1A/PPP2CA/RBL2/NFKB1/ATP6V0E2/ITGA2/ITGB4/FOXO1/JAG1/ATP6V 1E2/WNT3/WNT9A/ITGA6

4 ATP1A2/ITGB3/NCOA2/THRB/ITGAV/PIK3R3/BMP4/PLCE1/PLCG1/AKT2/PLCD4/PIK3CD/HIF1A/ATP1B1/HDAC2/T SC2/NCOA3/SLC2A1/MTOR/PRKACB/PDPK1/CTNNB1/MAPK1/RXRA/MED1/STAT1/SIN3A/RXRB/MDM2/CREBBP/ KRAS/NCOR1/NOTCH1/KAT2B/MAPK3/BAD/PLCB3/CCND1/PLCD3/AKT1/MED13/PIK3CA/GSK3B/NCOA1/PFKFB2/ PLCG2/PLCD1/ATP2A2/FOXO1/PLCB4/ATP1A3/GATA4

1.11E-14 2.01E-13 8.84E-14 SGPP2/GALC/PLPP3/CERK/PLPP2/UGCG/SPHK2/CERS6/DEGS1/SPTLC3/CERS1/GBA2/GLA/GBA/CERS2/NEU3/ KDSR/SGPL1/PLPP1/CERS4/ARSA/SGPP1/SMPD1/SPTLC1/SMPD2/B4GALT6/SPTLC2/SGMS1/GAL3ST1/SPHK1

2.00E-13 KDR/PDGFB/PDGFC/IL6/PDGFRA/PIK3R3/FGFR3/PLCG1/BAX/BCL2L11/AKT2/PDGFRB/PTEN/PIK3CD/IGF1R/EGF R/STAT3/EIF4EBP1/GAB1/MTOR/FOXO3/GAS6/SOS1/MAPK1/AXL/ERBB2/RPS6KB1/KRAS/MAPK3/BAD/SHC1/AK T1/PIK3CA/GSK3B/BCL2/JAK2/PLCG2/FGF2/PDGFA/ERBB3

1.83E-12 ELOVL4/HACD4/ACOT4/HADH/ELOVL5/HACD3/ECHS1/TECR/MECR/PPT1/ACAA2/ACOT7/ELOVL6/ELOVL7/ACOT 1/HACD2/ACOT2/HACD1/PPT2/ELOVL2/HSD17B12

1.07E-11 SH3GL3/AGAP2/AP2S1/PDGFRA/CYTH2/HSPA2/FGFR3/AP2A1/EPN1/ARF3/TGFBR1/RAB11FIP5/FGFR4/WASHC2 A/PLD1/SNX4/WASHC2C/IGF1R/RAB4A/EGFR/AP2B1/SNX32/EHD1/BIN1/SMURF1/GBF1/CHMP2A/CHMP3/ARPC1 B/AP2M1/PIP5K1C/SNX3/ASAP1/CLTA/RAB10/SH3GL1/RHOA/RAB7A/PIP5K1A/ACAP2/NEDD4/SNX2/MDM2/RAB8 A/SH3GLB1/KIF5B/RAB5C/ZFYVE9/RAB11FIP2/PRKCI/TGFBR2/VPS4A/PRKCZ/SNX1/VPS36/STAMBP/CAV1/CYTH 3/TRAF6/CBL/MVB12B/RAB31/ZFYVE16/CHMP5/PSD4/LDLR/CAV2/MVB12A/ARRB1/USP8/SNX6/AP2A2/EPN2/AM PH/SNX5/RAB35/EEA1/SPART

FASLG/SGK3/TNFSF10/S1PR1/AGAP2/IL6/FOXO6/MAPK8/CDKN1B/PIK3R3/TGFB2/IRS1/BCL2L11/TGFBR1/AKT2/ PTEN/ATM/PIK3CD/PRKAA2/TGFB1/IGF1R/PRKAG2/FBXO32/EGFR/PRKAB2/STAT3/PDPK1/FOXO3/INSR/SOS1/M APK1/MDM2/PRKAA1/CREBBP/KRAS/PRKAB1/CCNB1/MAPK3/ATG12/CCND1/TGFBR2/SIRT1/AKT1/PIK3CA/RBL2 IRS2/FOXO1/CAT/SLC2A4/PCK2/S1PR4

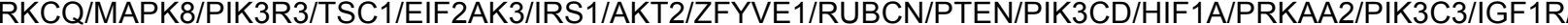
AMBRA1/TSC2/WIPI2/MTOR/PRKACB/PDPK1/PRKCD/MTMR3/MAPK1/RAB7A/ATG10/RPS6KB1/HMGB1/ATG2A/A TG2B/PRKAA1/KRAS/SH3GLB1/MAPK3/ATG7/CFLAR/BAD/ATG12/AKT1/TRAF6/PIK3CA/BECN1/CTSD/BCL2/MTM R4/DDIT4/ITPR1/PPP2CA/IRS2/ERN1

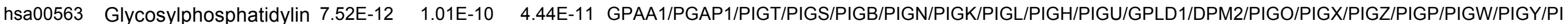

GV ositol (GPI)-anchor biosynthesis

hsa04151 PI3K-Akt signaling pathway
3.15E-11 4.00E-10

1.76E-10 PIK3CG/SPP1/LPAR4/KDR/LPAR3/PDGFB/PRLR/FASLG/ITGB3/COL1A1/LAMA1/TNC/IGF2/CSF1R/GNG2/PDGFC/ ERBB4/TLR2/TLR4/FGF1/SGK3/ITGAV/ITGB8/IL6/PDGFRA/LPAR2/F2R/CDKN1B/CCNE1/PPP2R1A/PIK3R3/FGFR3/ TSC1/IRS1/ITGB1/BCL2L11/AKT2/PDGFRB/THBS1/PTEN/FGFR4/GNB3/PIK3CD/PRKAA2/GNG12/IGF1R/YWHAH/E GFR/TSC2/GNB1/EIF4EBP1/MTOR/IFNAR1/PPP2R5B/PDPK1/FOXO3/CDK4/PTK2/INSR/SOS1/MAPK1/RXRA/RAC1 /ERBB2/RPS6KB1/CCND3/MDM2/PRKAA1/KRAS/PPP2R5C/MAPK3/PPP2R5A/BAD/FGFR1/CCND1/AKT1/PIK3CA/ 
GSK3B/BRCA1/BCL2/JAK2/DDIT4/PPP2CA/FLT4/RBL2/NFKB1/NR4A1/FGF2/ITGA2/MYB/ITGB4/PDGFA/PCK2/LPA R1/ERBB3/AREG/ITGA6/PIK3R5

hsa05163 Human cytomegalovirus infection

3.24E-11 4.00E-10

hsa04218 Cellular senescence

4.80E-11

hsa05215 Prostate cancer

1.01E-10 1.15E-09

1.31E-10 1.44E-09

Hepatocellular

carcinoma

hsa04146 Peroxisome

$1.58 \mathrm{E}-10 \quad 1.68 \mathrm{E}-09$

hsa05120 Epithelial cell signaling 3.19E-10 3.28E-09 in Helicobacter pylori infection

hsa04979 Cholesterol metabolism

hsa01522 Endocrine resistance

4.35E-10 4.34E-09

6.10E-10 5.89E-09 pathway

hsa04213 Longevity regulating pathway - multiple species

hsa04910 Insulin signaling pathway

hsa05167 Kaposi sarcoma-

1.25E-09 associated herpesvirus infection

hsa04012 ErbB signaling

1.57E-09 pathway

hsa05418 Fluid shear stress and 1.98E-09 atherosclerosis
$5.70 \mathrm{E}-10$

1.76E-10 NFATC4/FASLG/MAP2K6/ITGB3/ITPR2/CXCL8/GNG2/PTGER4/PTGER1/ITGAV/IL6/PDGFRA/PTGS2/PIK3R3/TSC1/ PTGER2/ADCY6/CALM3/BAX/RB1/AKT2/GNAI2/CASP3/GNB3/PIK3CD/BCAR1/GNG12/EGFR/STAT3/PPP3CB/IRF3/ TSC2/GNB1/E2F1/CRKL/EIF4EBP1/MTOR/PRKACB/GNAS/CRK/CDK4/NFKBIA/PTK2/GNAI1/CTNNB1/SOS1/MAPK1 /RAC1/CALR/RHOA/RPS6KB1/ROCK2/MDM2/KRAS/MAPK3/PLCB3/CCND1/CASP8/AKT1/PIK3CA/GSK3B/TNFRSF 1A/ADCY3/SP1/ELK1/ITPR1/NFKB1/PPP3CA/PTK2B/CCL2/PLCB4

2.50E-10 NFATC4/MAP2K6/ITPR2/CXCL8/IGFBP3/IL6/CCNE1/PIK3R3/TSC1/TGFB2/ETS1/CALM3/RB1/TGFBR1/AKT2/ZFP36 L2/PTEN/ATM/PIK3CD/MAPKAPK2/TGFB1/SERPINE1/CAPN2/PPP3CB/TSC2/E2F1/EIF4EBP1/MTOR/RBL1/FOXO3/ CDK4/ZFP36L1/MAPK1/CCNA2/SLC25A5/SLC25A6/SLC25A4/CCND3/MDM2/KRAS/CCNB1/MAPK3/CCND1/TGFBR 2/SIRT1/AKT1/PIK3CA/PPID/MCU/ITPR1/RBL2/NFKB1/PPP3CA/FOX01/TRPV4/GATA4

5.06E-10 PDGFB/PDGFC/AR/PDGFRA/CDKN1B/CCNE1/PIK3R3/RB1/AKT2/PDGFRB/PTEN/PIK3CD/IGF1R/EGFR/TCF7L2/E2 F1/MTOR/PDPK1/NFKBIA/CTNNB1/SOS1/MAPK1/ERBB2/MDM2/CREBBP/KRAS/MAPK3/BAD/FGFR1/CCND1/AKT1 /PIK3CA/GSK3B/BCL2/GSTP1/NKX3-1/NFKB1/FOXO1/PDGFA/INSRR

6.35E-10 GSTA1/WNT10B/IGF2/WNT5A/HMOX1/FZD5/SMARCC1/PIK3R3/TGFB2/PLCG1/BAX/RB1/TGFBR1/AKT2/PTEN/FZ D4/PIK3CD/FZD7/TGFB1/IGF1R/EGFR/TCF7L2/ARID1A/E2F1/GAB1/MTOR/APC/CDK4/MGST3/LRP6/CTNNB1/SOS 1/MAPK1/RPS6KB1/SMARCD1/KRAS/MAPK3/BAD/SHC1/CCND1/TGFBR2/AKT1/LRP5/GSTM4/PIK3CA/MGST2/GS K3B/MGST1/TXNRD1/WNT7B/PLCG2/ELK1/GSTP1/SMARCD3/NQO1/WNT3/WNT9A

7.40E-10 BAAT/ACOX2/HMGCLL1/ECH1/PEX2/ABCD3/ACOX3/ACSL4/PEX7/FAR2/SOD1/HMGCL/PEX13/ECI2/AGPS/ABCD1 /CRAT/GNPAT/FAR1/EHHADH/HACL1/DECR2/PEX19/ACSL1/DHRS4/PHYH/ACOX1/SCP2/MVK/HSD17B4/MLYCD/ CROT/CAT/SLC27A2/IDH1/NUDT7

1.44E-09 ATP6V0D2/CXCL1/CXCL8/ATP6V1H/JUN/TCIRG1/MAPK8/ATP6V1C1/PLCG1/CASP3/ATP6V0B/EGFR/PAK1/ATP6V 1G1/NFKBIA/ADAM17/ATP6V1B2/ATP6AP1/ATP6V0A1/ATP6V1E1/ATP6V1F/RAC1/PTPN11/ATP6V0D1/ATP6V1C2/ CSK/ATP6V0A2/ATP6V1A/PLCG2/NFKB1/ATP6V0E2/ATP6V1E2

1.91E-09 CD36/APOE/APOC1/PCSK9/ABCA1/CYP27A1/LRP1/LIPG/LCAT/PLTP/MYLIP/NPC1/STARD3/VAPB/SORT1/VAPA/N CEH1/LRPAP1/ANGPTL4/TSPO/LDLR/LIPA/SCARB1/OSBPL5/SOAT1/ANGPTL3

2 59E-09 JUN/MAPK8/CDKN1B/PIK3R3/ADCY6/BAX/RB1/AKT2/GPER1/PIK3CD/IGF1R/EGFR/NCOA3/E2F1/MTOR/PRKACB/ GNAS/CDK4/PTK2/SOS1/MAPK1/MED1/ERBB2/RPS6KB1/MDM2/KRAS/NCOR1/NOTCH1/CARM1/MAPK3/BAD/SH C1/CCND1/AKT1/PIK3CA/ADCY3/BCL2/SP1/JAG1

3.29E-09 IRAK3/FASLG/JUN/MAPK8/RIPK2/PIK3R3/IRAK2/IRS1/RAP1B/PLCG1/CALM3/BAX/AKT2/PIK3CD/MAPKAPK2/CRK L/GAB1/CRK/PDPK1/PRKCD/FOXO3/NFKBIA/SORT1/SOS1/MAPK1/CAMK2G/RPS6KA3/ABL1/RAC1/RHOA/PTPN1 1/KRAS/MAPK3/BAD/SHC1/NFKBIB/AKT1/IRAK1/TRAF6/PIK3CA/GSK3B/BCL2/PLCG2/NFKB1

4.40E-09 EIF4EBP2/HSPA2/PIK3R3/IRS1/ADCY6/AKT2/PIK3CD/PRKAA2/HDAC2/IGF1R/PRKAG2/PRKAB2/MTOR/PRKACB/S OD1/FOXO3/INSR/RPS6KB1/PRKAA1/KRAS/PRKAB1/SIRT1/AKT1/PIK3CA/ADCY3/IRS2/FOXO1/CAT/CRYAB

40E-09 PPARGC1A/LIPE/MAPK8/PIK3R3/TSC1/IRS1/CALM3/SOCS2/AKT2/PIK3CD/PRKAA2/PRKAG2/ACACA/PRKAB2/PD E3B/TSC2/CRKL/EIF4EBP1/MTOR/PRKACB/INPPL1/CRK/PDPK1/TRIP10/INSR/SOS1/MAPK1/SREBF1/RPS6KB1/P RKAA1/KRAS/PRKAB1/MAPK3/BAD/SHC1/PRKCI/PRKCZ/AKT1/PIK3CA/CBL/GSK3B/ACACB/INPP5K/ELK1//RS2/F OXO1/SLC2A4/PCK2

4.71E-09 PIK3CG/NFATC4/PDGFB/CXCL1/MAP2K6/ITPR2/CXCL8/GNG2/JUN/IL6/MAPK8/PTGS2/PIK3R3/PLCG1/CALM3/BA X/RB1/PREX1/AKT2/CASP3/GNB3/PIK3CD/HIF1A/MAPKAPK2/PIK3C3/GNG12/STAT3/PPP3CB/IRF3/GNB1/E2F1/M TOR/IFNAR1/CDK4/NFKBIA/CTNNB1/MAPK1/STAT1/RAC1/CREBBP/KRAS/MAPK3/TICAM1/CCND1/CASP8/AKT1// CAM1/PIK3CA/GSK3B/TNFRSF1A/BECN1/JAK2/PLCG2/ITPR1/MICB/NFKB1/PPP3CA/FGF2/PIK3R5

78E-09 ERBB4/JUN/MAPK8/CDKN1B/PIK3R3/PLCG1/AKT2/PIK3CD/EGFR/PAK1/CRKL/EIF4EBP1/GAB1/MTOR/CRK/PTK2/ SOS1/MAPK1/CAMK2G/ABL1/ERBB2/RPS6KB1/KRAS/MAPK3/BAD/SHC1/AKT1/PIK3CA/CBL/GSK3B/STAT5B/PLC G2/ELK1/ERBB3/AREG

1.61E-08 7.07E-09 MEF2C/KDR/GSTA1/PDGFB/MAP2K6/ITGB3/JUN/ITGAV/HMOX1/SDC2/MAPK8/PIK3R3/BMP4/EDN1/ACVR2B/SDC 4/CALM3/AKT2/PIK3CD/PRKAA2/MGST3/DUSP1/PTK2/CTNNB1/RAC1/RHOA/PRKAA1/SDC1/PRKCZ/AKT1/GSTM4 ICAV1/ICAM1/PIK3CA/MGST2/TNFRSF1A/MGST1/BCL2/GPC1/CAV2/GSTP1/ASS1/NFKB1/PDGFA/NQO1/CCL2/TH BD/TRPV4 
hsa05142 Chagas disease (American trypanosomiasis)

hsa05220 Chronic myeloid leukemia

hsa04015 Rap1 signaling pathway

hsa04211 Longevity regulating pathway

hsa05224 Breast cance

$1.58 \mathrm{E}-08 \quad 1.14 \mathrm{E}-07$

hsa00565 Ether lipid metabolism 2.03E-08

1.42E-07

hsa00071 Fatty acid degradation 2.50E-08

1.71E-07

hsa04666 Fc gamma R-mediated 3.54E-08 phagocytosis

hsa04150 mTOR signaling pathway

$6.53 \mathrm{E}-08$

2.38E-07

4.29E-07

hsa00561 Glycerolipid metabolism

hsa04371 Apelin signaling pathway

hsa05152 Tuberculosis

hsa04510 Focal adhesion

1.37E-07 8.33E-07

hsa04062 Chemokine signaling pathway

hsa05323 Rheumatoid arthritis

1.05E-06

hsa05226 Gastric cancer

$8.70 \mathrm{E}-08 \quad 5.60 \mathrm{E}-07$

1.08E-07 6.83E-07
3.16E-07 ITGB2/ATP6V0D2/TLR2/TLR4/ATP6V1H/VDR/IL6/TCIRG1/MAPK8/RIPK2/IRAK2/TGFB2/CALM3/BAX/AKT2/CASP3/T LR6/SPHK2/TGFB1/PIK3C3/ATP6V0B/MALT1/PPP3CB/NFYA/ATP6AP1/ATP6V0A1/MAPK1/CAMK2G/STAT1/RHOA RAB7A/CREBBP/MAPK3/BAD/RAB5C/CASP8/AKT1/ATP6V0D1/IRAK1/TRAF6/TNFRSF1A/CTSD/BCL2/ATP6V0A2/J AK2/NFYB/NFKB1/IL18/PPP3CA/IL12A/CEBPB/EEA1/SPHK1

3.66E-07 SPP1/KDR/PDGFB/ITGB3/COL1A1/LAMA1/TNC/PDGFC/JUN/ITGAV/ITGB8/PDGFRA/MAPK8/PIK3R3/ARHGAP35/R AP1B/ITGB1/AKT2/PDGFRB/THBS1/PTEN/PIK3CD/BCAR1/IGF1R/TLN1/CAPN2/EGFR/PAK1/CRKL/CRK/PDPK1/PT K2/CTNNB1/PIP5K1C/SOS1/MAPK1/RAC1/RHOA/ERBB2/ROCK2/CCND3/MAPK3/BAD/SHC1/CCND1/AKT1/CAV1/P IK3CA/GSK3B/BCL2/CAV2/ELK1/FLT4/ITGA2/ITGB4/PDGFA/ITGA6

.06E-07 PIK3CG/CXCL6/CXCL1/CXCL5/CXCL8/GNG2/PIK3R3/RAP1B/ADCY6/GSK3A/PREX1/AKT2/GNAI2/GNB3/PIK3CD/B CAR1/GNG12/PAK1/STAT3/GNB1/CRKL/PRKACB/CRK/PRKCD/FOXO3/NFKBIA/PTK2/GNAI1/SOS1/MAPK1/STAT1/ RAC1/RHOA/ROCK2/KRAS/MAPK3/BAD/SHC1/PLCB3/NFKBIB/PRKCZ/AKT1/PIK3CA/GSK3B/ADCY3/STAT5B/JAK 2/ARRB1/NFKB1/PTK2B/CCL2/PLCB4/TIAM1/CXCL16/PIK3R5

62E-07 ITGB2/CXCL6/TNFSF11/ATP6V0D2/CXCL1/CXCL5/CXCL8/TLR2/TLR4/ATP6V1H/JUN/IL6/TCIRG1/ATP6V1C1/TGFB 2/TGFB1/ATP6V0B/ATP6V1G1/ATP6V1B2/ATP6AP1/ATP6V0A1/ATP6V1E1/ATP6V1F/ATP6V0D1/ICAM1/ATP6V1C2 IATP6V0A2/ATP6V1A/IL18/ATP6V0E2/ATP6V1E2/CCL2/TNFRSF11A

2.09E-07 1.18E-06 5.19E-07 ABCB1/SHH/WNT10B/WNT5A/FGF1/CDKN1B/FZD5/CCNE1/PIK3R3/TGFB2/BAX/RB1/TGFBR1/AKT2/FZD4/PIK3CD /FZD7/TGFB1/EGFR/TCF7L2/E2F1/GAB1/MTOR/APC/LRP6/CTNNB1/SOS1/MAPK1/RXRA/ERBB2/RPS6KB1/RXRB/ 
hsa05161 Hepatitis B

hsa04010 MAPK signaling pathway

3.44E-07 1.88E-06

hsa05235 PD-L1 expression and 3.47E-07 PD-1 checkpoint pathway in cancer

hsa05211 Renal cell carcinoma

hsa05110 Vibrio cholerae infection

hsa05210 Colorectal cancer

hsa05135 Yersinia infection

hsa04917 Prolactin signaling pathway hsa04923 Regulation of lipolysis
in adipocytes

hsa04370 VEGF signaling pathway

hsa05212 Pancreatic cancer

hsa05214 Glioma

hsa04810 Regulation of actin cytoskeleton

hsa00640 Propanoate metabolism

hsa04611 Platelet activation

9.92E-07 4.51E-06

1.04E-06 4.59E-06

hsa04922 Glucagon signaling

1.04E-06 4.59E-06 pathway

hsa00650 Butanoate metabolism 1.40E-06

hsa04625

4.05E-07 2.16E-06

4.39E-07 2.30E-06

4.95E-07 2.55E-06

5.15E-07 2.61E-06

5.70E-07 2.84E-06

3.58E-06

$3.62 \mathrm{E}-06$

7.73E-07 3.62E-06

7.73E-07 3.62E-06

8.27E-07 3.81E-06
NFATC4/FASLG/MAP2K6/CXCL8/TLR2/TLR4/JUN/IL6/MAPK8/CCNE1/PIK3R3/HSPG2/TGFB2/BAX/RB1/TGFBR1/A KT2/CASP3/PIK3CD/TGFB1/STAT3/IRF3/E2F1/IFNAR1/NFKBIA/ATP6AP1/SOS1/MAPK1/STAT1/CCNA2/TAB2/CRE BBP/KRAS/MAPK3/BAD/TICAM1/TGFBR2/CASP8/AKT1/IRAK1/TRAF6/PIK3CA/STAT5B/BCL2/JAK2/ELK1/NFKB1/P TK2B/PCNA

8.26E-07 MEF2C/KDR/PDGFB/PLA2G4A/FASLG/MAP2K6/PLA2G4C/DUSP10/IGF2/CSF1R/PDGFC/ERBB4/FGF1/JUN/CACN A1H/PDGFRA/MAPK8/HSPA2/FGFR3/PPP5C/TGFB2/RAP1B/TGFBR1/AKT2/PDGFRB/CASP3/FGFR4/RASGRP3/M APKAPK2/MAP4K4/TGFB1/PLA2G4B/GNG12/IGF1R/RAPGEF2/JUND/EGFR/PAK1/NFKB2/PPP3CB/CRKL/PRKACB/ CRK/DUSP1/INSR/SOS1/MAPK1/RPS6KA3/RAC1/TAB2/DAXX/ERBB2/KRAS/MAX/MAPK3/RASA2/FGFR1/TGFBR2/ AKT1/IRAK1/TRAF6/TNFRSF1A/PPM1A/MAPKAPK3/ELK1/FLT4/ARRB1/NFKB1/NR4A1/PPP3CA/FGF2/PDGFA/MA PT/ERBB3/AREG

8.26E-07 MAP2K6/TLR2/TLR4/JUN/PRKCQ/PIK3R3/PLCG1/AKT2/PTEN/PIK3CD/HIF1A/EGFR/STAT3/PPP3CB/MTOR/NFKB A/MAPK1/STAT1/RPS6KB1/PTPN11/KRAS/MAPK3/TICAM1/NFKBIB/AKT1/TRAF6/PIK3CA/JAK2/NFKB1/CSNK2A2/ PPP3CA/CD274

9.48E-07 PDGFB/JUN/PIK3R3/EGLN2/ARNT2/TGFB2/ETS1/RAP1B/AKT2/PIK3CD/HIF1A/TGFB1/PAK1/SLC2A1/CRKL/GAB1/ CRK/SOS1/MAPK1/RAC1/PTPN11/CREBBP/KRAS/MAPK3/BAD/AKT1/PIK3CA

1.01E-06 ATP6V0D2/ATP6V1H/TCIRG1/ATP6V1C1/PLCG1/ATP6V0B/PRKACB/GNAS/ATP6V1G1/ATP6V1B2/ATP6AP1/ATP6 V0A1/ATP6V1E1/ATP6V1F/ATP6V0D1/ATP6V1C2/ADCY3/ATP6V0A2/ATP6V1A/PLCG2/ATP6V0E2/ATP6V1E2

1.12E-06 JUN/MAPK8/PIK3R3/TGFB2/BAX/BCL2L11/TGFBR1/AKT2/CASP3/PIK3CD/TGFB1/EGFR/TCF7L2/MTOR/APC/CTN NB1/SOS1/MAPK1/RAC1/RHOA/RPS6KB1/KRAS/MAPK3/BAD/CCND1/TGFBR2/AKT1/PIK3CA/GSK3B/BCL2/AREG

1.15E-06 MAP2K6/CXCL8/TLR4/JUN/IL6/MAPK8/PIK3R3/PLCG1/ITGB1/AKT2/PIK3CD/BCAR1/CASP1/IRF3/CRKL/CRK/NFKB A/PTK2/PIP5K1C/MAPK1/RPS6KA3/RAC1/TAB2/RHOA/PIP5K1A/ROCK2/MAPK3/TICAM1/AKT1/IRAK1/TRAF6/PIK3 CA/GSK3B/BAIAP2/NFKB1/IL18/PTK2B/PYCARD/CCL2

1.25E-06 TNFSF11/PRLR/LHB/SLC2A2/MAPK8/CISH/PIK3R3/SOCS2/AKT2/PIK3CD/SOCS5/STAT3/FOXO3/SOS1/MAPK1/ST AT1/KRAS/MAPK3/SHC1/CCND1/AKT1/PIK3CA/GSK3B/STAT5B/JAK2/NFKB1/TNFRSF11A

1.57E-06 AQP7/ADORA1/PTGS1/LIPE/PTGS2/PIK3R3/IRS1/ADCY6/AKT2/GNAI2/PIK3CD/PDE3B/PRKACB/GNAS/INSR/GNAI 1/MGLL/AKT1/PIK3CA/ADCY3/ABHD5/PNPLA2/IRS2

1.59E-06 KDR/PLA2G4A/PLA2G4C/PTGS2/PIK3R3/PLCG1/AKT2/SPHK2/PIK3CD/MAPKAPK2/PLA2G4B/PPP3CB/PTK2/MAP K1/RAC1/KRAS/MAPK3/BAD/AKT1/PIK3CA/MAPKAPK3/PLCG2/PPP3CA/SPHK1

1.59E-06 MAPK8/PIK3R3/TGFB2/BAX/RB1/TGFBR1/AKT2/PIK3CD/PLD1/TGFB1/EGFR/STAT3/E2F1/MTOR/CDK4/MAPK1/ST AT1/RAC1/ERBB2/RPS6KB1/KRAS/MAPK3/BAD/CCND1/TGFBR2/AKT1/PIK3CA/NFKB1

1.59E-06 PDGFB/PDGFRA/PIK3R3/PLCG1/CALM3/BAX/RB1/AKT2/PDGFRB/PTEN/PIK3CD/IGF1R/EGFR/E2F1/MTOR/CDK4/ SOS1/MAPK1/CAMK2G/MDM2/KRAS/MAPK3/SHC1/CCND1/AKT1/PIK3CA/PLCG2/PDGFA

F2/LPAR4/ITGB2/PDGFB/ITGB3/PDGFC/FGF1/ITGAV/ITGB8/GSN/PDGFRA/LPAR2/F2R/PIK3R3/FGFR3/ARHGAP3 5/ITGB1/PDGFRB/FGFR4/PIK3CD/BCAR1/PIP4K2A/GNG12/EGFR/PAK1/PIKFYVE/CRKL/IQGAP1/MSN/CRK/PFN1/ APC/ARPC1B/PTK2/PIP4K2B/PIP5K1C/SOS1/MAPK1/RAC1/RHOA/PIP5K1A/ROCK2/KRAS/MAPK3/FGFR1/PIP4K2 C/PIK3CA/BAIAP2/EZR/PFN2/FGF2/ITGA2/ITGB4/PDGFA/LPAR1/ITGA6/TIAM1/INSRR

1.98E-06 ACSS3/ACOX3/ACACA/ECHS1/ACAT2/EHHADH/ECHDC1/ALDH6A1/DLD/ACACB/ACADS/HIBCH/ACOX1/MLYCD/P CCB/ACSS2/ABAT

2.02E-06 F2/PIK3CG/PLA2G4A/ITGB3/ITPR2/COL1A1/PLA2G4C/PTGS1/TBXAS1/F2R/PIK3R3/TBXA2R/ARHGAP35/RAP1B/A DCY6/ITGB1/AKT2/GNAI2/PIK3CD/PLA2G4B/TLN1/PRKACB/GNAS/GNAI1/MAPK1/RHOA/ROCK2/MAPK3/PLCB3/P RKCI/PRKCZ/AKT1/PIK3CA/ADCY3/PLCG2/ITPR1/ITGA2/PLCB4/PIK3R5

2.02E-06 PPARGC1A/ITPR2/CPT1C/SLC2A2/CALM3/AKT2/PRKAA2/PRKAG2/ACACA/PRKAB2/PDE3B/PPP3CB/CPT1B/PFK L/SLC2A1/SIK2/PRKACB/GNAS/CAMK2G/PKM/PRKAA1/CREBBP/PRKAB1/PPP4R3B/PLCB3/SIRT1/AKT1/ACACB/ PPARA/ITPR1/PPP3CA/FOXO1/PCK2/CPT1A/PLCB4

6.09E-06 2.68E-06 ACSM5/HMGCLL1/BDH1/ACSM3/HADH/BDH2/ECHS1/HMGCL/ACAT2/EHHADH/AACS/ACADS/HMGCS1/ALDH5A1/

8.01E-06 3.52E-06 NFATC4/ITPR2/JUN/IL6/MAPK8/PTGS2/PIK3R3/CALM3/AKT2/PIK3CD/MAPKAPK2/CASP1/MALT1/PAK1/NFKB2/PP P3CB/PRKCD/NFKBIA/MAPK1/STAT1/RHOA/MDM2/PTPN11/KRAS/MAPK3/CASP8/AKT1/PIK3CA/PLCG2/ITPR1/NF 
signaling pathway

hsa04934 Cushing syndrome

hsa05145 Toxoplasmosis

1.92E-06

8.01E-06

$2.21 \mathrm{E}-06$

9.09E-06

3.95E-06 1.60E-05

4.78E-06 1.89E-05 isoleucine degradatio

hsa05160 Hepatitis C

hsa04966 Collecting duct acid secretion

hsa05230 Central carbon metabolism in cancer

Ras signaling pathway

5.63E-06

$6.20 \mathrm{E}-06$

2.36E-05

6.32E-06 2.38E-05

hsa04142 Lysosome

7.38E-06 2.75E-05

hsa05162 Measles

hsa04930 Type II diabetes mellitus

hsa05213 Endometrial cancer

hsa04928 Parathyroid hormone synthesis. secretion and action virus 1 infection

hsa04932 Non-alcoholic fatty liver 9.48E-06 3.33Edisease (NAFLD)

hsa05133 Pertussis

1.23E-05

4.26E-05

hsa00100 Steroid biosynthesis

hsa04066 HIF-1 signaling
KB1/PPP3CA/IL12A/PYCARD

3.52E-06 ITPR2/AGT/PBX1/WNT10B/WNT5A/CACNA1H/CDKN1B/FZD5/CCNE1/RAP1B/ADCY6/RB1/GNAI2/FZD4/FZD7/AHR/ EGFR/TCF7L2/E2F1/PRKACB/GNAS/APC/CDK4/GNAI1/CTNNB1/MAPK1/CAMK2G/NCEH1/MAPK3/PLCB3/CCND1/ GSK3B/ADCY3/SP1/LDLR/WNT7B/ITPR1/USP8/NR4A1/SCARB1/WNT3/WNT9A/CYP11A1/PLCB4/POMC

3.52E-06 PIK3CG/MAP2K6/LAMA1/LY96/TLR2/TLR4/MAPK8/HSPA2/TGFB2/ITGB1/AKT2/GNAI2/CASP3/TGFB1/STAT3/PDPK 1/NFKBIA/GNAI1/MAPK1/STAT1/TAB2/MAPK3/BAD/NFKBIB/CASP8/AKT1/IRAK1/TRAF6/TNFRSF1A/BCL2/JAK2/LD LR/NFKB1/IL12A/ITGA6/PIK3R5

3.99E-06 PIK3R3/PLCG1/BAX/RB1/AKT2/PIK3CD/EGFR/STAT3/E2F1/PDPK1/FOXO3/CDK4/SOS1/MAPK1/RXRA/ERBB2/RX RB/KRAS/MAPK3/BAD/CCND1/AKT1/PIK3CA/STAT5B/PLCG2

7.05E-06 PDGFB/PDGFC/FGF1/PDGFRA/PIK3R3/BAX/RB1/AKT2/PDGFRB/PTEN/PIK3CD/IGF1R/EGFR/E2F1/CDK4/MAPK1/ MDM2/KRAS/MAPK3/BAD/FGFR1/CCND1/AKT1/PIK3CA/FGF2/PDGFA

7.29E-06 HMGCLL1/HADH/AUH/ECHS1/ACAD8/HMGCL/ACAT2/ALDH3A2/ACAA2/EHHADH/ALDH6A1/AACS/IVD/DLD/ACAD S/HMGCS1/HIBCH/PCCB/ACADSB/ABAT

8.32E-06 FASLG/CLDN4/PPP2R1A/PIK3R3/EIF2AK3/BAX/RB1/AKT2/CASP3/PIK3CD/YWHAH/EGFR/STAT3/IRF3/E2F1/IFNA R1/CDK4/NFKBIA/PIAS1/CTNNB1/SOS1/MAPK1/RXRA/STAT1/KRAS/MAPK3/CFLAR/BAD/TICAM1/CCND1/CASP8/ AKT1/TRAF6/PIK3CA/GSK3B/TNFRSF1A/PPARA/NR1H3/LDLR/PPP2CA/NFKB1/CD81/CLDN1/SCARB1

9.06E-06 ATP6V0D2/TCIRG1/ATP6V1C1/ATP6V1G1/ATP6V1B2/ATP6V0A1/ATP6V1E1/ATP6V1F/ATP6V0D1/ATP6V1C2/ATP 6V0A2/ATP6V1A/ATP6V0E2/ATP6V1E2

9.56E-06 PDGFRA/SLC2A2/PIK3R3/PDK1/FGFR3/AKT2/PDGFRB/PTEN/PIK3CD/HIF1A/EGFR/PFKL/SLC2A1/MTOR/G6PD/M APK1/PKM/ERBB2/KRAS/MAPK3/FGFR1/AKT1/PIK3CA/IDH1/SIRT3

1.04E-05 PLA1A/KDR/PDGFB/PLA2G4A/FASLG/PLA2G4C/IGF2/CSF1R/GNG2/RGL1/PDGFC/FGF1/PDGFRA/MAPK8/PIK3R3 /FGFR3/PLCE1/ETS1/RAP1B/PLCG1/CALM3/AKT2/PDGFRB/FGFR4/GNB3/RASGRP3/PIK3CD/PLD1/PLA2G4B/GN G12/IGF1R/EGFR/PAK1/GNB1/GAB1/PRKACB/INSR/SOS1/MAPK1/ABL1/RAC1/RHOA/PTPN11/KRAS/MAPK3/BAD/ RAB5C/RASA2/SHC1/FGFR1/AKT1/PIK3CA/PLCG2/ELK1/FLT4/NFKB1/FGF2/PDGFA/TIAM1

1.05E-05 CTSH/ATP6V0D2/GALC/ATP6V1H/TCIRG1/PSAP/LAPTM4B/ABCA2/PLA2G15/SCARB2/CTSA/ATP6V0B/NPC1/HEX B/CD68/GLA/ATP6AP1/SORT1/ATP6V0A1/GBA/PPT1/AP3S1/CLTA/ATP6V0D1/AP3M2/ARSA/CTSD/CLN3/ATP6V0A 2/AP1M2/GM2A/LIPA/SMPD1/FUCA1/LITAF/TPP1/PPT2

1.21E-05 FASLG/TLR2/TLR4/JUN/IL6/MAPK8/CDKN1B/HSPA2/CCNE1/PIK3R3/EIF2AK3/TNFAIP3/BAX/AKT2/CASP3/PIK3CD/ STAT3/IRF3/IFNAR1/MSN/CDK4/NFKBIA/STAT1/TAB2/CCND3/RACK1/BAD/CCND1/NFKBIB/CASP8/AKT1/IRAK1/T RAF6/PIK3CA/GSK3B/STAT5B/BCL2/NFKB1/CSNK2A2/IL12A

8.38E-06 3.08E-05 1.36E-05 ABCC8/SLC2A2/MAPK8/PIK3R3/IRS1/SOCS2/PRKCE/PIK3CD/MTOR/PRKCD/INSR/MAPK1/PKM/MAPK3/PRKCZ/PI $\mathrm{K} 3 \mathrm{CA} / \mathrm{RS} 2 / \mathrm{SLC} 2 \mathrm{~A} 4 / \mathrm{KCN} 111$

1.39E-05 PIK3R3/BAX/AKT2/PTEN/PIK3CD/EGFR/TCF7L2/PDPK1/FOXO3/APC/CTNNB1/SOS1/MAPK1/ERBB2/KRAS/MAPK3 /BAD/CCND1/AKT1/PIK3CA/GSK3B/ELK1

1.39E-05 MEF2C/TNFSF11/ITPR2/VDR/ADCY6/GNAI2/PLD1/JUND/EGFR/PRKACB/GNAS/LRP6/MMP15/GNAI1/MAPK1/RXR A/RHOA/RXRB/MAPK3/PLCB3/FGFR1/LRP5/GATA3/ADCY3/BCL2/SP1/BGLAP/ITPR1/ARRB1/PDE4D/PDE4B/NR4A 2/PLCB4

1.39E-05 ITGB2/NFATC4/JUN/IL6/MAPK8/CCNE1/PIK3R3/TGFB2/ETS1/ADCY6/BAX/RB1/TGFBR1/AKT2/PTEN/ATM/PIK3CD/ TGFB1/TLN1/NFKB2/PPP3CB/SLC2A1/E2F1/PRKACB/CDK4/NFKBIA/MAPK1/CCNA2/CALR/SLC25A5/SLC25A6/FD PS/SLC25A4/CCND3/KAT5/CREBBP/KRAS/KAT2B/MAPK3/CCND1/TGFBR2/AKT1/FOSL1/ICAM1/VAC14/PIK3CA/T NFRSF1A/ADCY3/STAT5B/CRTC3/TSPO/ELK1/NFYB/NFKB1/PPP3CA/RAN

1.46E-05 CEBPA/FASLG/CXCL8/JUN/IL6/MAPK8/PIK3R3/LEPR/EIF2AK3/IRS1/GSK3A/BAX/BCL2L11/AKT2/CASP3/PIK3CD/P RKAA2/TGFB1/PRKAG2/ADIPOR1/PRKAB2/XBP1/INSR/RXRA/RAC1/SREBF1/PRKAA1/PRKAB1/CASP8/AKT1/PIK3 CA/NDUFS6/GSK3B/TNFRSF1A/PPARA/ADIPOR2/NR1H3/NFKB1/IRS2/NDUFAB1/ERN1/CYP2E1

1.87E-05 ITGB2/CXCL6/CXCL5/CXCL8/LY96/TLR4/JUN/IL6/MAPK8/CALM3/ITGB1/GNAI2/CASP3/CASP1/IRF3/GNAI1/MAPK1 /RHOA/MAPK3/TICAM1/IRAK1/TRAF6/NFKB1/IL12A/PYCARD/IRF8

1.40E-05 4.79E-05 2.11E-05 CEL/DHCR24/LSS/SQLE/DHCR7/MSMO1/EBP/CYP51A1/LIPA/CYP2R1/SOAT1

1.71E-05 5.80E-05 2.55E-05 TLR4/HMOX1/IL6/CDKN1B/PIK3R3/PDK1/EGLN2/EDN1/PLCG1/AKT2/PIK3CD/HIF1A/IGF1R/SERPINE1/EGFR/STA 
pathway

hsa01524 Platinum drug resistance

hsa04913 Ovarian steroidogenesis

hsa05221 Acute myeloid

leukemia

hsa05170 Human

immunodeficiency

virus 1 infection

hsa04024 cAMP signaling pathway

hsa04380 Osteoclast differentiation

1.76E-05

5.91E-05

$247 \mathrm{E}-05-820 \mathrm{E}-05$

2.75E-05

9.05E-05

3.05E-05 9.91E-05

4.02E-05 1.29E-04

4.27E-05

1.36E-04

hsa04721 Synaptic vesicle cycle

hsa04210 Apoptosis

7.33E-05

2.29E-04

2.49E-04

TNF signaling pathway $8.06 \mathrm{E}-05$

hsa05222 Small cell lung cancer 8.15E-05

hsa00900 Terpenoid backbone biosynthesis

hsa05144 Malaria

hsa04921 Oxytocin signaling pathway

hsa04926 Relaxin signaling pathway

hsa04217 Necroptosis

hsa05134 Legionellosis

hsa04912 GnRH signaling pathway

hsa04350 TGF-beta signaling pathway
T3/PFKL/SLC2A1/EIF4EBP1/MTOR/INSR/MAPK1/CAMK2G/ERBB2/RPS6KB1/CREBBP/MAPK3/GAPDH/AKT1/PIK3 CA/BCL2/PLCG2/NFKB1

2.60E-05 GSTA1/FASLG/PIK3R3/BAX/AKT2/CASP3/ATM/PIK3CD/PDPK1/MGST3/MAPK1/ERBB2/MDM2/MAPK3/BAD/CASP8/ AKT1/GSTM4/PIK3CA/MGST2/BRCA1/MGST1/BCL2/GSTP1/ABCC2

3.60E-05 PLA2G4A/PLA2G4C/AKR1C3/LHB/BMP6/CYP1A1/PTGS2/ADCY6/PLA2G4B/IGF1R/CYP1B1/PRKACB/GNAS/INSR/ ADCY3/LDLR/ACOT2/SCARB1/CYP11A1

3.98E-05 CEBPA/CSF1R/PIK3R3/AKT2/RARA/PPARD/PIK3CD/TCF7L2/STAT3/EIF4EBP1/MTOR/SOS1/MAPK1/RPS6KB1/KR AS/MAPK3/BAD/PIM1/CCND1/AKT1/PIK3CA/STAT5B/NFKB1

4.36E-05 NFATC4/FASLG/MAP2K6/ITPR2/GNG2/TLR2/TLR4/JUN/MAPK8/PIK3R3/PLCG1/CALM3/BAX/AKT2/GNAI2/CASP3/A TM/GNB3/PIK3CD/GNG12/PAK1/PPP3CB/IRF3/GNB1/CRKL/MTOR/CRK/NFKBIA/PTK2/GNAI1/MAPK1/RAC1/TAB2/ CALR/RPS6KB1/KRAS/CCNB1/MAPK3/BAD/CASP8/AKT1/IRAK1/TRAF6/PIK3CA/TNFRSF1A/BCL2/AP1M2/PLCG2/I TPR1/NFKB1/PPP3CA/PTK2B/TNFRSF1B

5.68E-05 HTR1B/EDN2/ATP1A2/SSTR1/ADORA1/LIPE/JUN/SOX9/MAPK8/RAPGEF4/F2R/PIK3R3/EDN1/PTGER2/PLCE1/RA P1B/ADCY6/CALM3/AKT2/GNAI2/ACOX3/PIK3CD/ATP1B1/PLD1/PDE3B/PAK1/PRKACB/GNAS/NFKBIA/GNAI1/MAP K1/CAMK2G/RAC1/RHOA/ROCK2/CREBBP/MAPK3/BAD/AKT1/ATP2B1/PIK3CA/ADCY3/PPARA/ACOX1/NFKB1/AT P2A2/PTCH1/PDE4D/OXTR/PDE4B/ATP1A3/TIAM1/POMC

98E-05 TNFSF11/MAP2K6/ITGB3/CSF1R/JUN/FOSB/MAPK8/PIK3R3/TGFB2/TGFBR1/AKT2/PIK3CD/TGFB1/JUND/NFKB2/ PPP3CB/IFNAR1/JUNB/NFKBIA/MAPK1/STAT1/RAC1/FHL2/TAB2/MAPK3/TGFBR2/AKT1/FOSL1/TRAF6/PIK3CA/T NFRSF1A/PLCG2/NFKB1/PPP3CA/PPARG/TNFRSF11A

8.67E-05 SYT1/ATP6V0D2/ATP6V1H/CPLX1/AP2S1/TCIRG1/STX1A/ATP6V1C1/AP2A1/ATP6V0B/AP2B1/ATP6V1G1/AP2M1/ ATP6V1B2/ATP6V0A1/ATP6V1E1/ATP6V1F/CLTA/ATP6V0D1/ATP6V1C2/ATP6V0A2/ATP6V1A/ATP6V0E2/AP2A2/A TP6V1E2

1.00E-04 CTSH/FASLG/ITPR2/JUN/TNFSF10/MAPK8/PIK3R3/EIF2AK3/BAX/BCL2L11/AKT2/CASP3/ATM/PIK3CD/CAPN2/PD PK1/NFKBIA/MAPK1/DAXX/KRAS/DAB2IP/MAPK3/CFLAR/BAD/CASP8/AKT1/PIK3CA/TNFRSF1A/CTSD/BCL2/AIFM 1/DFFA/PARP1/ITPR1/PTPN13/NFKB1/ERN1

1.09E-04 CXCL6/CXCL1/MAP2K6/CXCL5/JUN/IL6/MAPK8/PTGS2/PIK3R3/EDN1/TNFAIP3/AKT2/CASP3/PIK3CD/JUNB/NFKBI A/MAPK1/TAB2/DAB2IP/MAPK3/CFLAR/DNM1L/CASP8/AKT1/ICAM1/PIK3CA/TNFRSF1A/NFKB1/JAG1/CEBPB/CCL 2/TNFRSF1B

2.49E-04 1.10E-04 LAMA1/ITGAV/CDKN1B/CCNE1/PTGS2/PIK3R3/BAX/ITGB1/RB1/AKT2/CASP3/PTEN/PIK3CD/E2F1/CDK4/NFKBIA/ PTK2/RXRA/RXRB/MAX/CCND1/AKT1/TRAF6/PIK3CA/BCL2/NFKB1/ITGA2/ITGA6

1.14E-04 NUS1/PCYOX1/IDI1/ACAT2/FDPS/GGPS1/MVD/HMGCR/PDSS2/HMGCS1/MVK

1.22E-04 ITGB2/KLRK1/CD36/CXCL8/TLR2/TLR4/IL6/SDC2/LRP1/TGFB2/THBS1/TGFB1/SDC1/ICAM1/IL18/IL12A/CD81/CCL

$9.28 \mathrm{E}-05 \quad 2.78 \mathrm{E}-04$

9.97E-05 2.96E-04 1.30E-04 PIK3CG/MEF2C/NFATC4/PLA2G4A/ITPR2/PLA2G4C/JUN/CD38/PTGS2/ADCY6/CALM3/GNAI2/PRKAA2/PLA2G4B/ PRKAG2/EGFR/PRKAB2/PPP3CB/PRKACB/GNAS/GNAI1/MAPK1/CAMK2G/RHOA/ROCK2/PRKAA1/KRAS/PRKAB1 /EEF2K/MAPK3/EEF2/PLCB3/CCND1/ADCY3/ELK1/ITPR1/PPP3CA/OXTR/PLCB4/PIK3R5

1.43E-04 4.20E-04 1.85E-04 NOS1/COL1A1/GNG2/JUN/MAPK8/PIK3R3/EDN1/ADCY6/TGFBR1/AKT2/GNAI2/GNB3/PIK3CD/TGFB1/GNG12/EGF R/GNB1/PRKACB/GNAS/NFKBIA/GNAI1/SOS1/MAPK1/KRAS/MAPK3/SHC1/PLCB3/TGFBR2/PRKCZ/AKT1/PIK3CA/ ADCY3/ARRB1/NFKB1/PLCB4

1.78E-04 5.18E-04 2.27E-04 PLA2G4A/FASLG/PLA2G4C/TLR4/TNFSF10/MAPK8/TNFAIP3/BAX/GLUL/CASP1/PLA2G4B/CAPN2/STAT3/IFNAR1/ CHMP2A/CHMP3/CAMK2G/STAT1/PPIA/SLC25A5/SLC25A6/HMGB1/SLC25A4/CFLAR/DNM1L/TICAM1/VPS4A/CAS P8/TNFRSF1A/STAT5B/GLUD1/BCL2/PPID/AIFM1/CHMP5/JAK2/PARP1/SMPD1/H2AFY/PYCARD/H2AFZ

2.03E-04 5.86E-04 2.58E-04 ITGB2/CXCL1/CXCL8/TLR2/TLR4/IL6/HSPA2/CASP3/CASP1/HSF1/NFKB2/NFKBIA/VCP/CASP8/NFKB1/IL18/IL12A/ PYCARD/EEF1A2

2.13E-04 6.09E-04 2.68E-04 PLA2G4A/MAP2K6/ITPR2/PLA2G4C/LHB/GNRH1/JUN/MAPK8/ADCY6/CALM3/PLD1/PLA2G4B/EGFR/PRKACB/GN AS/PRKCD/SOS1/MAPK1/CAMK2G/KRAS/MAPK3/PLCB3/ADCY3/ELK1/ITPR1/PTK2B/PLCB4

2.58E-04 7.32E-04 3.22E-04 BMP2/DCN/BMP6/INHBB/SMAD6/PPP2R1A/BMP4/TGFB2/ACVR2B/TGFBR1/THBS1/TGFB1/FBN1/SMURF1/RBL1/ MAPK1/RHOA/RPS6KB1/CREBBP/MAPK3/ZFYVE9/TGFBR2/ZFYVE16/SP1/ID3/PPP2CA/INHBA 
hsa04620 Toll-like receptor signaling pathway

hsa04750 Inflammatory media regulation of TRP channels

hsa02010 ABC transporters

3.48E-04 9.61E-04

hsa00061 Fatty acid biosynthesis 3.87E-04 1.06E-03

hsa04145 Phagosome

3.94E-04 1.07E-03

hsa04915 Estrogen signaling pathway

hsa04710 Circadian rhythm

hsa05203 Viral carcinogenesis

4.94E-04 1.33E-03

8.48E-04 2.24E-03

hsa04916 Melanogenesis

hsa04662 B cell receptor signaling pathway

8.94E-04 2.34E-03

1.03E-03 2.68E-03

Th17 cell differentiation 1.04E-03

hsa00590 Arachidonic acid 1.07E-03 metabolism

hsa04310 Wnt signaling pathway 1.13E-03

hsa04664 Fc epsilon RI signaling 1.14E-03 pathway

hsa00072 Synthesis and degradation of ketone bodies

hsa04020 Calcium signaling pathway

1.18E-03 2.93E-03

hsa04925 Aldosterone synthesis and secretion

hsa04975 Fat digestion and absorption

hsa04961 Endocrine and other factor-regulated calcium reabsorption

hsa04022 cGMP-PKG signaling

1.25E-03 3.06E-03

1.30E-03 3.15E-03
$1.14 \mathrm{E}-03 \quad 284 \mathrm{E}-03$

3.35E-04 SPP1/MAP2K6/CXCL8/LY96/TLR2/TLR4/JUN/L6/MAPK8/PIK3R3/AKT2/TLR6/PIK3CD/IRF3/IFNAR1/NFKBIA/MAPK1 ISTAT1/RAC1/TAB2/MAPK3/TICAM1/CASP8/AKT1/IRAK1/TRAF6/PIK3CA/NFKB1/IL12A

3.91E-04 PLA2G4A/MAP2K6/ITPR2/PLA2G4C/PTGER4/PRKCQ/MAPK8/PIK3R3/PTGER2/ADCY6/PLCG1/CALM3/PRKCE/PIK 3CD/F2RL1/PLA2G4B/PRKACB/GNAS/PRKCD/CAMK2G/PLCB3/PIK3CA/ADCY3/PLCG2/ITPR1/ALOX12/PLCB4/TR PV4

4.22E-04 ABCA13/ABCB4/ABCB1/ABCA8/ABCC8/ABCA12/ABCA1/ABCC3/ABCA2/ABCD3/ABCA7/ABCC1/ABCD1/ABCA3/AB CG1/ABCC2

4.65E-04 OLAH/ACSL4/ACACA/MECR/MCAT/ACSL1/ACACB/CBR4/ACSBG1

4.69E-04 NOS1/ITGB2/ATP6V0D2/CD36/ITGB3/TLR2/TLR4/ATP6V1H/ITGAV/TCIRG1/ATP6V1C1/ITGB1/THBS1/TLR6/PIK3C3 /ATP6V0B/PIKFYVE/ATP6V1G1/ATP6V1B2/ATP6AP1/ATP6V0A1/ATP6V1E1/ATP6V1F/RAC1/CALR/RAB7A/RAB5C/ ATP6V0D1/ATP6V1C2/ATP6V0A2/ATP6V1A/STX12/ATP6V0E2/ITGA2/SCARB1/ATP6V1E2/OLR1/EEA1

5.83E-04 ITPR2/NCOA2/JUN/HSPA2/PIK3R3/ADCY6/CALM3/AKT2/GNAI2/GPER1/RARA/PIK3CD/EGFR/NCOA3/PRKACB/GN AS/PRKCD/GNAI1/SOS1/MAPK1/FKBP4/KRAS/MAPK3/SHC1/PLCB3/AKT1/PIK3CA/ADCY3/CTSD/BCL2/NCOA1/SP 1/ITPR1/PLCB4/POMC

9.29E-04 RORA/RORB/RORC/PRKAA2/PRKAG2/PRKAB2/PRKAA1/PRKAB1/NR1D1/CRY1/ARNTL/NPAS2

9.85E-04 ATP6V0D2/JUN/GSN/CDKN1B/HDAC9/CCNE1/PIK3R3/HDAC5/BAX/RB1/CASP3/PIK3CD/MAPKAPK2/HDAC2/YWH AH/NFKB2/STAT3/IRF3/RBL1/PRKACB/CDK4/NFKBIA/MAPK1/RAC1/CCNA2/PKM/RHOA/CCND3/MDM2/UBE3A/CR EBBP/KRAS/KAT2B/MAPK3/BAD/RASA2/CCND1/CASP8/ATP6V0D1/VAC14/PIK3CA/STAT5B/RBL2/NFKB1/SNW1/H IST1H2BJ

1.03E-03 WNT10B/WNT5A/FZD5/EDN1/ADCY6/CALM3/GNAI2/FZD4/FZD7/TCF7L2/PRKACB/GNAS/GNAI1/CTNNB1/MAPK1/ CAMK2G/CREBBP/KRAS/MAPK3/PLCB3/GSK3B/ADCY3/WNT7B/WNT3/WNT9A/PLCB4/POMC

1.18E-03 JUN/PIK3R3/AKT2/RASGRP3/PIK3CD/MALT1/PPP3CB/INPPL1/NFKBIA/SOS1/MAPK1/RAC1/KRAS/MAPK3/NFKBIB IAKT1/PIK3CA/GSK3B/PLCG2/NFKB1/PPP3CA/CD81/INPP5D

1.18E-03 RORA/RORC/JUN/PRKCQ/IL6/MAPK8/PLCG1/TGFBR1/RARA/HIF1A/TGFB1/AHR/STAT3/PPP3CB/MTOR/NFKBIA/ MAPK1/RXRA/STAT1/RXRB/MAPK3/TGFBR2/NFKBIB/GATA3/STAT5B/JAK2/NFKB1/PPP3CA

1.21E-03 PTGIS/PLA2G4A/GGT1/PLA2G4C/AKR1C3/PTGS1/TBXAS1/GPX3/LTA4H/PTGS2/GPX1/PLA2G4B/CBR1/PTGES3/P TGES2/ALOX12/GGT5/ALOX12B/CYP2E1

1.25E-03 NFATC4/GPC4/WNT10B/WNT5A/SERPINF1/SFRP1/JUN/MAPK8/FZD5/FZD4/PPARD/FZD7/TCF7L2/PPP3CB/PRKA CB/APC/LRP6/CTNNB1/CAMK2G/RAC1/RHOA/ROCK2/CCND3/CREBBP/PLCB3/CCND1/FOSL1/LRP5/DKK1/GSK3 B/TBL1X/WNT7B/CSNK2A2/PPP3CA/PORCN/WNT3/WNT9A/PLCB4

1.25E-03 ALOX5AP/PLA2G4A/MAP2K6/PLA2G4C/MAPK8/PIK3R3/PLCG1/AKT2/PIK3CD/PLA2G4B/PDPK1/SOS1/MAPK1/RA C1/KRAS/MAPK3/AKT1/PIK3CA/PLCG2/INPP5D

1.25E-03 HMGCLL1/BDH1/BDH2/HMGCL/ACAT2/HMGCS1

1.29E-03 NOS1/ITPR2/ERBB4/PTGFR/P2RX7/CD38/PTGER1/CACNA1H/PDGFRA/F2R/TBXA2R/PLCE1/PLCG1/CALM3/PDG FRB/PLCD4/SPHK2/EGFR/PPP3CB/P2RX4/PRKACB/GNAS/CAMK2G/SLC25A5/SLC25A6/ERBB2/TPCN1/SLC25A4/ PLCB3/PLCD3/ATP2B1/ADCY3/MCU/LTB4R2/PLCG2/ITPR1/PPP3CA/PLCD1/ATP2A2/PTK2B/OXTR/ERBB3/PLCB4/ SPHK1 CB3/ATP2B1/ADCY3/LDLR/ITPR1/NR4A1/SCARB1/NR4A2/CYP11A1/PLCB4/ATP1A3/POMC

1.28E-03 3.10E-03 1.36E-03 CD36/NPC1L1/ABCA1/CEL/DGAT2/PLPP3/PLPP2/DGAT1/AGPAT1/ACAT2/PLPP1/SLC27A4/GOT2/SCARB1

1.38E-03 ATP1A2/VDR/AP2S1/AP2A1/ADCY6/ATP1B1/AP2B1/PRKACB/GNAS/AP2M1/CLTA/PLCB3/ATP2B1/AP2A2/PLCB4/A TP1A3

1.37E-03 3.29E-03 1.44E-03 PIK3CG/ADRA2A/MEF2C/NFATC4/ATP1A2/ITPR2/ADORA1/IRS1/ADCY6/CALM3/AKT2/GNAI2/PRKCE/ATP1B1/PD 
pathway

hsa04924 Renin secretion

1.39E-03

hsa04960 Aldosterone-regulated 1.40E-03 sodium reabsorption

hsa05206 MicroRNAs in cancer

hsa00410 beta-Alanine metabolism

hsa05169 Epstein-Barr virus infection

hsa05014 Amyotrophic lateral sclerosis (ALS)

hsa04064 NF-kappa B signaling pathway

hsa05164 Influenza A

hsa04520 Adherens junction

hsa04550 Signaling pathways regulating pluripotency of stem cells

hsa04657 IL-17 signaling pathway

hsa05217 Basal cell carcinoma

hsa00604 Glycosphingolipid biosynthesis - ganglio series

hsa05140 Leishmaniasis

hsa04390 Hippo signaling pathway

hsa05219 Bladder cancer

hsa04730 Long-term depression

1.43E-03

3.36E-03

$1.51 \mathrm{E}-03 \quad 3.51 \mathrm{E}-03$

1.55E-03 3.57E-03

1.65E-03

3.77E-03

1.71E-03 3.88E-03

1.95E-03 4.39E-03

2.44E-03 5.46E-03

2.65E-03 5.88E-03

2.81E-03

6.17E-03

2.81E-03

6.17E-03

2.92E-03 6.36E-03

6.36E-03

3.46E-03

7.47E-03

3.80E-03

8.15E-03

3.96E-03

4.01E-03

hsa04927 Cortisol synthesis and 4.08E-03 secretion

hsa04115 p53 signaling pathway 5.72E-03

hsa04976 Bile secretion

5.72E-03 1.19E-02
E3B/PPP3CB/INSR/GNAI1/MAPK1/RHOA/SLC25A5/SLC25A6/ROCK2/SLC25A4/MAPK3/BAD/PLCB3/AKT1/ATP2B1/ ADCY3/ITPR1/IRS2/PPP3CA/ATP2A2/ADRA2C/PLCB4/ATP1A3/GATA4/PIK3R5

1.45E-03 EDN2/ITPR2/AGT/ADORA1/PTGER4/EDN1/PTGER2/ADCY6/CALM3/GNAI2/PDE3B/PPP3CB/PRKACB/GNAS/GNA 1/PLCB3/ITPR1/PPP3CA/ACE/PLCB4

1.45E-03 NR3C2/ATP1A2/PIK3R3/IRS1/PIK3CD/ATP1B1/PDPK1/INSR/MAPK1/KRAS/MAPK3/PIK3CA/ATP1A3

1.47E-03 ABCB1/PDGFB/ITGB3/SOX4/TNC/HMOX1/DNMT3A/PDGFRA/CDKN1B/CCNE1/PTGS2/PIK3R3/FGFR3/TGFB2/IRS1 /PLCG1/BCL2L11/PDGFRB/THBS1/CASP3/PTEN/ATM/DNMT3B/PRKCE/PIK3CD/ABCC1/EGFR/IGF2BP1/STAT3/E2 F1/CRKL/EZH2/CYP1B1/MTOR/CRK/APC/SOS1/MAPK1/ABL1/VIM/RHOA/ERBB2/MDM2/CREBBP/KRAS/NOTCH1/ MAPK3/PIM1/SHC1/CCND1/SIRT1/PIK3CA/BRCA1/BCL2/PLCG2/DDIT4/EZR/NFKB1/IRS2/SLC45A3/PDGFA/WNT3/ BCL2L2/ERBB3

1.54E-03 ACOX3/ALDH3B1/ECHS1/ALDH3A2/EHHADH/ALDH6A1/ACADS/HIBCH/ACOX1/MLYCD/ABAT/ALDH3A1

1.57E-03 MAP2K6/TLR2/JUN/IL6/MAPK8/CDKN1B/CCNE1/PIK3R3/TNFAIP3/BAX/BCL2L11/RB1/AKT2/CASP3/PIK3CD/HDAC 2/NFKB2/STAT3/IRF3/E2F1/IFNAR1/CDK4/NFKBIA/STAT1/RAC1/CCNA2/VIM/SIN3A/TAB2/CALR/NEDD4/CCND3/M DM2/CCND1/NFKBIB/CASP8/AKT1/ICAM1/IRAK1/TRAF6/PIK3CA/BCL2/PLCG2/NFKB1/SNW1

1.66E-03 NOS1/MAP2K6/GPX1/BAX/CASP3/CASP1/PPP3CB/SOD1/RAC1/DAXX/BAD/TNFRSF1A/BCL2/PPP3CA/CAT/TNFR SF1B

1.71E-03 TNFSF11/CXCL8/LY96/TLR4/PRKCQ/PTGS2/PLCG1/TNFAIP3/ATM/MALT1/NFKB2/NFKBIA/TAB2/CFLAR/TICAM1/I CAM1/IRAK1/TRAF6/TNFRSF1A/TRIM25/BCL2/PARP1/PLCG2/NFKB1/CSNK2A2/TNFRSF11A

1.93E-03 FASLG/CXCL8/TLR4/TNFSF10/IL6/PIK3R3/BAX/AKT2/CASP3/PIK3CD/CASP1/IRF3/IFNAR1/CDK4/NFKBIA/MAPK1/ STAT1/SLC25A5/SLC25A6/FDPS/SLC25A4/CCND3/PABPN1/CREBBP/MAPK3/TICAM1/NFKBIB/CASP8/AKT1/ICAM 1/PIK3CA/TNFRSF1A/TRIM25/JAK2/NFKB1/IL18/IL12A/PYCARD/CCL2

2.40E-03 SNAI2/SNAI1/TGFBR1/IGF1R/EGFR/TCF7L2/IQGAP1/INSR/CTNNB1/MAPK1/RAC1/RHOA/ERBB2/CREBBP/MAPK3 /FGFR1/TGFBR2/BAIAP2/YES1/CSNK2A2

2.58E-03 WNT10B/WNT5A/INHBB/FZD5/PIK3R3/FGFR3/BMP4/ACVR2B/AKT2/FGFR4/FZD4/PIK3CD/FZD7/IGF1R/STAT3/RE ST/APC/CTNNB1/MAPK1/KRAS/MAPK3/FGFR1/AKT1/PIK3CA/GSK3B/JAK2/WNT7B/ID3/INHBA/FGF2/WNT3/KLF4/ WNT9A

2.71E-03 CXCL6/CXCL1/CXCL5/CXCL8/JUN/FOSB/IL6/MAPK8/PTGS2/TNFAIP3/CASP3/JUND/NFKBIA/MAPK1/TAB2/MAPK3/ CASP8/FOSL1/TRAF6/GSK3B/NFKB1/CEBPB/CCL2/MUC5AC

2.71E-03 SHH/BMP2/WNT10B/WNT5A/FZD5/BMP4/BAX/FZD4/FZD7/TCF7L2/APC/CTNNB1/GSK3B/SMO/WNT7B/PTCH1/WN T3/WNT9A

2.80E-03 ST6GALNAC5/ST3GAL2/ST3GAL5/ST6GALNAC6/HEXB/ST6GALNAC4/B4GALNT1

3.28E-03 ITGB2/TLR2/TLR4/JUN/PTGS2/TGFB2/ITGB1/TGFB1/NFKBIA/MAPK1/STAT1/TAB2/MAPK3/NFKBIB/IRAK1/TRAF6/J AK2/ELK1/NFKB1/IL12A

58E-03 AFP/ITGB2/BMP2/SNAI2/WNT10B/WNT5A/BMP6/FGF1/TEAD2/FZD5/PPP2R1A/BMP4/TGFB2/TGFBR1/FZD4/FZD7/ TGFB1/SERPINE1/YWHAH/TCF7L2/AJUBA/APC/CTNNB1/CCND3/PRKCI/CCND1/TGFBR2/PRKCZ/GSK3B/WNT7B/ PPP2CA/YAP1/WNT3/WNT9A/AREG

8.44E-03 3.71E-03 CXCL8/FGFR3/RB1/THBS1/EGFR/E2F1/CDK4/MAPK1/ERBB2/MDM2/KRAS/MAPK3/CCND1

8.48E-03 3.73E-03 NOS1/PLA2G4A/ITPR2/PLA2G4C/PPP2R1A/GNAI2/PLA2G4B/IGF1R/GNAS/GNAI1/MAPK1/KRAS/MAPK3/PLCB3/IT PR1/PPP2CA/PLCB4

8.57E-03 3.77E-03 ITPR2/AGT/PBX1/CACNA1H/ADCY6/PRKACB/GNAS/NCEH1/PLCB3/ADCY3/SP1/LDLR/ITPR1/NR4A1/SCARB1/CY P11A1/PLCB4/POMC

5.22E-03 IGFBP3/CCNE1/BAX/THBS1/CASP3/PTEN/ATM/SERPINE1/SESN2/TSC2/SESN3/CDK4/CCND3/MDM2/CCNB1/CC ND1/CASP8/BCL2/AIFM2

5.22E-03 NR1H4/ABCB4/ABCB1/BAAT/ATP1A2/ABCC3/ADCY6/ATP1B1/SLC2A1/PRKACB/GNAS/RXRA/NCEH1/HMGCR/AD 
hsa04540 Gap junction

6.21E-03

hsa04728 Dopaminergic synapse 6.28E-03

$1.28 \mathrm{E}-02$

$1.29 \mathrm{E}-02$

hsa04726 Serotonergic synapse

$6.54 \mathrm{E}-03 \quad 1.33 \mathrm{E}-02$

hsa00120 Primary bile acid biosynthesis

hsa05131 Shigellosis

6.79E-03 1.37E-02

hsa05100 Bacterial invasion of epithelial cells

hsa05146 Amoebiasis

hsa04621 NOD-like receptor signaling pathway

hsa01200 Carbon metabolism

hsa04670 Leukocyte transendothelial migration

hsa04725 Cholinergic synapse

hsa04660 T cell receptor signaling pathway

hsa00480 Glutathione metabolism

hsa04136 Autophagy - other

hsa05216 Thyroid cancer

hsa00380 Tryptophan

metabolism

hsa00430 Taurine and hypotaurine metabolism

hsa00620 Pyruvate metabolism

hsa04911 Insulin secretion

hsa00220 Arginine biosynthesis

hsa00360 Phenylalanine metabolism

hsa00830 Retinol metabolism
$6.81 \mathrm{E}-03 \quad 1.37 \mathrm{E}-02$

$7.84 \mathrm{E}-03 \quad 1.56 \mathrm{E}-02$

$7.88 \mathrm{E}-03 \quad 1.56 \mathrm{E}-02$

$7.93 \mathrm{E}-03 \quad 1.56 \mathrm{E}-02$

8.31E-03 1.62E-02

8.76E-03 1.69E-02

8.76E-03

1.69E-02

1.10E-02

2.11E-02

1.15E-02

2.20E-02

1.23E-02 2.32E-02

1.31E-02 2.46E-02

1.36E-02 2.54E-02

1.37E-02 2.55E-02

1.95E-02 3.59E-02

1.95E-02 3.59E-02

2.41E-02 4.40E-02

2.72E-02 4.94E-02

2.73E-02 4.94E-02
5.62E-03 PDGFB/ITPR2/PDGFC/PDGFRA/GJA1/ADCY6/PDGFRB/GNAI2/EGFR/PRKACB/GNAS/GNAI1/SOS1/MAPK1/KRAS/ MAPK3/PLCB3/ADCY3/ITPR1/PDGFA/LPAR1/PLCB4

5.65E-03 MAOB/ITPR2/GNG2/MAPK8/PPP2R1A/GSK3A/CALM3/AKT2/GNAI2/GNB3/GNG12/PPP3CB/GNB1/PPP2R5B/PRKA CB/GNAS/GNAI1/CAMK2G/PPP2R5C/KIF5B/PPP2R5A/PLCB3/AKT1/GSK3B/COMT/ITPR1/PPP2CA/PPP3CA/ARNT L/PLCB4

5.84E-03 HTR1B/PLA2G4A/MAOB/ITPR2/PLA2G4C/GNG2/PTGS1/PTGS2/APP/GNAI2/CASP3/GNB3/PLA2G4B/GNG12/GNB1 /PRKACB/GNAS/DUSP1/GNAI1/MAPK1/KRAS/MAPK3/PLCB3/ITPR1/ALOX12/PLCB4/ALOX12B

6.01E-03 BAAT/ACOX2/CYP27A1/CYP39A1/SCP2/HSD17B4/HSD3B7

6.01E-03 HCLS1/CXCL8/MAPK8/RIPK2/ITGB1/CRKL/CRK/PFN1/ARPC1B/NFKBIA/MAPK1/ABL1/RAC1/ROCK2/MAPK3/NFKBI B/NFKB1/PFN2

6.86E-03 HCLS1/PIK3R3/TGGB1/PIK3CD/BCAR1/CRKL/GAB1/CRK/ARPC1B/PTK2/CTNNB1/RAC1/CLTA/RHOA/SHC1/CAV1/P IK3CA/CBL/CAV2

6.86E-03 ITGB2/CXCL1/COL1A1/LAMA1/CXCL8/TLR2/TLR4/IL6/PIK3R3/TGFB2/CASP3/PIK3CD/TGFB1/PRKACB/GNAS/PTK 2/RAB7A/RAB5C/PLCB3/PIK3CA/NFKB1//L12A/PLCB4

6.86E-03 CARD16/CXCL1/ITPR2/CXCL8/TLR4/P2RX7/JUN/IL6/MAPK8/RIPK2/TNFAIP3/CASP1/IRF3/GSDMD/IFNAR1/PRKCD /NFKBIA/MAPK1/STAT1/TAB2/RHOA/MAPK3/DNM1L/TICAM1/ATG12/PLCB3/NFKBIB/CASP8/TXNIP/TRAF6/BCL2/ MCU/ITPR1/NFKB1/IL18/PYCARD/CCL2/PLCB4

7.14E-03 ACOX3/ECHS1/PFKL/ME1/G6PD/ACAT2/PKM/ADH5/EHHADH/GAPDH/ALDH6A1/DLD/PC/ACADS/GLUD1/HIBCH/A COX1/GOT1/PCCB/PSPH/GOT2/ACSS2/CAT/IDH1/CPS1/RGN/GLDC

7.44E-03 ITGB2/CLDN4/THY1/RAPGEF4/PIK3R3/ARHGAP35/RAP1B/PLCG1/ITGB1/GNAI2/PIK3CD/BCAR1/MSN/PTK2/GNAI 1/CTNNB1/RAC1/RHOA/ROCK2/PTPN11/ICAM1/PIK3CA/PLCG2/EZR/CLDN1/PTK2B

7.44E-03 PIK3CG/ITPR2/GNG2/PIK3R3/ADCY6/AKT2/GNAI2/GNB3/PIK3CD/GNG12/GNB1/PRKACB/GNAI1/MAPK1/CAMK2G/ KRAS/MAPK3/PLCB3/AKT1/PIK3CA/ADCY3/BCL2/JAK2/ITPR1/PLCB4/PIK3R5

9.26E-03 JUN/PRKCQ/MAPK8/PIK3R3/PLCG1/AKT2/PIK3CD/MALT1/PAK1/PPP3CB/PDPK1/CDK4/NFKBIA/SOS1/MAPK1/RH OA/KRAS/MAPK3/NFKBIB/AKT1/PIK3CA/GSK3B/NFKB1/PPP3CA

9.67E-03 GSTA1/GGT1/GPX3/GPX1/GPX4/GGT7/MGST3/G6PD/GSTM4/MGST2/MGST1/GCLC/GSTP1/IDH1/GGT5

1.02E-02 PIK3C3/WIPI2/MTOR/ATG10/ATG2A/ATG2B/ATG7/ATG12/BECN1/PPP2CA

1.08E-02 BAX/TCF7L2/CTNNB1/MAPK1/RXRA/RXRB/KRAS/MAPK3/NCOA4/CCND1/PPARG

1.12E-02 MAOB/CYP1A1/HADH/ECHS1/CYP1B1/KMO/ACAT2/ALDH3A2/EHHADH/DLD/GCDH/CAT

1.12E-02 CDO1/BAAT/GGT1/GGT7/GGT5

1.58E-02 ACOT12/ACACA/ME1/ACAT2/PKM/ALDH3A2/DLD/PC/ACACB/ACSS2/PCK2

1.58E-02 RIMS2/ATP1A2/KCNN4/PCLO/ABCC8/SLC2A2/RAPGEF4/STX1A/ADCY6/ATP1B1/SLC2A1/PRKACB/GNAS/CAMK2 G/PLCB3/TRPM4/ADCY3/KCNJ11/PLCB4/ATP1A3

1.93E-02 NOS1/GLUL/GLUD1/ASS1/GOT1/GOT2/CPS1

2.17E-02 MAOB/MIF/ALDH3B1/GOT1/GOT2/ALDH3A1

2.17E-02 UGT2B15/LRAT/CYP3A5/DHRS3/SDR16C5/CYP2S1/CYP1A1/DGAT1/RDH10/ADH5/PNPLA4/DHRS4/RETSAT/RDH 5/CYP26B1/RPE65 
Supplementary table 4. Molar percentages of fatty acid species from total lipids, their sums and ratios of different fatty acids in control and knock-down ANGPTL3 (shANGPTL3) IHH cells. Determined by gas chromatography (mean \pm SD, $\mathrm{n}=6$ ). Statistically significant differences between the groups are marked with asterisks. ${ }^{*} p<0.05,{ }^{* *} p<0.01,{ }^{* * *}$ $p<0.001$ (two-tailed Student's t-test). Marking DMA refers to alkenyl chain derived dimethyl acetal, SAT=saturated, MUFA=monounsaturated, PUFA=polyunsaturated, UNSAT=unsaturated.

\begin{tabular}{|c|c|c|c|c|c|c|}
\hline \multirow{2}{*}{$\frac{\text { Fatty acid }}{14: 00}$} & \multicolumn{3}{|c|}{ control } & \multicolumn{3}{|c|}{ shANGPTL3 } \\
\hline & 2.34 & \pm & 0.14 & 2.46 & \pm & 0.08 \\
\hline $15: 0^{*}$ & 0.36 & \pm & 0.03 & 0.42 & \pm & 0.05 \\
\hline DMA16:0** & 2.33 & \pm & 0.14 & 2.01 & \pm & 0.13 \\
\hline $16: 0$ * & 27.30 & \pm & 0.51 & 27.96 & \pm & 0.45 \\
\hline $16: 1 n-9$ & 4.23 & \pm & 0.13 & 4.23 & \pm & 0.12 \\
\hline $16: 1 n-7^{* *}$ & 1.87 & \pm & 0.18 & 1.61 & \pm & 0.12 \\
\hline $17: 0^{* * *}$ & 0.47 & \pm & 0.02 & 0.54 & \pm & 0.03 \\
\hline $17: 1 n-8$ & 0.57 & \pm & 0.03 & 0.57 & \pm & 0.04 \\
\hline DMA18:0 & 1.74 & \pm & 0.10 & 1.78 & \pm & 0.15 \\
\hline $18: 0$ & 12.35 & \pm & 0.31 & 12.51 & \pm & 0.41 \\
\hline $18: 1 n-9^{* *}$ & 22.58 & \pm & 0.50 & 21.34 & \pm & 0.76 \\
\hline $18: 1 n-7$ & 4.67 & \pm & 0.06 & 4.71 & \pm & 0.06 \\
\hline $18: 2 n-6$ & 0.94 & \pm & 0.05 & 0.93 & \pm & 0.06 \\
\hline $20: 0$ & 0.36 & \pm & 0.02 & 0.36 & \pm & 0.04 \\
\hline $20: 1 n-11$ & 0.45 & \pm & 0.29 & 0.77 & \pm & 0.90 \\
\hline $20: 1 n-9^{*}$ & 0.73 & \pm & 0.05 & 0.82 & \pm & 0.09 \\
\hline $20: 3 n-9$ & 2.17 & \pm & 0.64 & 1.54 & \pm & 0.31 \\
\hline $20: 3 n-6^{* *}$ & 1.18 & \pm & 0.04 & 1.31 & \pm & 0.06 \\
\hline $20: 4 n-6^{* * *}$ & 4.95 & \pm & 0.13 & 5.46 & \pm & 0.24 \\
\hline $20: 5 n-3^{* * *}$ & 0.59 & \pm & 0.04 & 0.98 & \pm & 0.05 \\
\hline $22: 0^{* * *}$ & 0.61 & \pm & 0.02 & 0.55 & \pm & 0.02 \\
\hline $22: 1 n-9$ & 0.36 & \pm & 0.13 & 0.32 & \pm & 0.06 \\
\hline $22: 4 n-6^{* * *}$ & 0.42 & \pm & 0.04 & 0.52 & \pm & 0.03 \\
\hline $22: 5 n-3^{* * *}$ & 0.80 & \pm & 0.09 & 1.09 & \pm & 0.04 \\
\hline $24: 0^{* * *}$ & 1.29 & \pm & 0.05 & 1.16 & \pm & 0.03 \\
\hline $22: 6 n-3^{*}$ & 3.43 & \pm & 0.13 & 3.21 & \pm & 0.15 \\
\hline $24: 1 n-9$ & 0.89 & \pm & 0.06 & 0.84 & \pm & 0.02 \\
\hline SUM SAT & 45.09 & \pm & 0.64 & 45.97 & \pm & 0.79 \\
\hline SUM MUFA** & 36.35 & \pm & 0.45 & 35.21 & \pm & 0.65 \\
\hline SUM PUFA & 14.49 & \pm & 0.87 & 15.03 & \pm & 0.74 \\
\hline SUM n-6*** & 7.50 & \pm & 0.19 & 8.22 & \pm & 0.30 \\
\hline SUM n-3** & 4.82 & \pm & 0.25 & 5.27 & \pm & 0.21 \\
\hline UNSAT/SAT & 1.13 & \pm & 0.03 & 1.09 & \pm & 0.03 \\
\hline$n-3 / n-6$ & 0.64 & \pm & 0.03 & 0.64 & \pm & 0.01 \\
\hline DMAs & 4.07 & \pm & 0.24 & 3.79 & \pm & 0.29 \\
\hline
\end{tabular}


Supplementary table 5. Molar percentages of phosphatidylcholine (PC) species in control and ANGPTL3 knock-down (shANGPTL3) IHH cells. Determined by ESI-MS/MS (mean $\pm S D, n=6$ ). Statistically significant differences between the groups are marked with asterisks; ${ }^{*} p<0.05,{ }^{* *} p<0.01,{ }^{* * *} p<0.001$ (two-tailed Student's t-test).

\begin{tabular}{|c|c|c|c|c|c|c|}
\hline PC & \multicolumn{3}{|c|}{ control } & \multicolumn{3}{|c|}{ shANGPTL3 } \\
\hline $28: 0^{* *}$ & 0.16 & \pm & 0.01 & 0.19 & \pm & 0.01 \\
\hline $30: 0^{* * *}$ & 3.36 & \pm & 0.10 & 3.99 & \pm & 0.21 \\
\hline C30:0alkyl & 0.59 & \pm & 0.04 & 0.57 & \pm & 0.03 \\
\hline $30: 1$ & 0.16 & \pm & 0.05 & 0.21 & \pm & 0.08 \\
\hline C30:1alkyl & 0.05 & \pm & 0.02 & 0.04 & \pm & 0.01 \\
\hline $32: 0^{* * \star}$ & 5.29 & \pm & 0.34 & 7.05 & \pm & 0.19 \\
\hline C32:0alkyl & 1.91 & \pm & 0.13 & 1.95 & \pm & 0.16 \\
\hline $32: 1$ & 10.81 & \pm & 0.59 & 11.56 & \pm & 0.63 \\
\hline 32:1alky| & 2.15 & \pm & 0.13 & 1.69 & \pm & 0.04 \\
\hline $32: 2^{*}$ & 1.59 & \pm & 0.09 & 1.72 & \pm & 0.07 \\
\hline 34:0alkyl* & 0.98 & \pm & 0.05 & 0.88 & \pm & 0.08 \\
\hline $34: 1^{* *}$ & 25.71 & \pm & 0.30 & 24.42 & \pm & 0.84 \\
\hline $34: 1$ alkyl** & 4.14 & \pm & 0.19 & 3.80 & \pm & 0.12 \\
\hline $34: 2$ & 5.24 & \pm & 0.49 & 5.17 & \pm & 0.11 \\
\hline 34:2alkyl** & 0.79 & \pm & 0.05 & 0.69 & \pm & 0.03 \\
\hline $34: 3^{*}$ & 0.43 & \pm & 0.03 & 0.35 & \pm & 0.07 \\
\hline 34:3alkyl & 0.11 & \pm & 0.03 & 0.09 & \pm & 0.01 \\
\hline $34: 5$ & 0.88 & \pm & 0.46 & 1.31 & \pm & 0.22 \\
\hline $35: 5^{* *}$ & 0.24 & \pm & 0.02 & 0.28 & \pm & 0.02 \\
\hline $36: 1^{* * *}$ & 2.98 & \pm & 0.21 & 2.20 & \pm & 0.21 \\
\hline 36:1alky|** & 1.29 & \pm & 0.05 & 1.17 & \pm & 0.06 \\
\hline $36: 2^{*}$ & 8.45 & \pm & 0.27 & 7.86 & \pm & 0.44 \\
\hline 36:2alky| $\left.\right|^{* *}$ & 1.28 & \pm & 0.08 & 1.13 & \pm & 0.05 \\
\hline $36: 3$ & 2.46 & \pm & 0.21 & 2.44 & \pm & 0.12 \\
\hline 36:3alkyl** & 0.53 & \pm & 0.05 & 0.43 & \pm & 0.03 \\
\hline $36: 4^{* * *}$ & 1.85 & \pm & 0.10 & 2.09 & \pm & 0.08 \\
\hline 36:4alkyl|** & 0.97 & \pm & 0.04 & 0.80 & \pm & 0.07 \\
\hline $36: 5^{\star * \star}$ & 0.49 & \pm & 0.03 & 0.79 & \pm & 0.04 \\
\hline $38: 0^{*}$ & 0.39 & \pm & 0.03 & 0.35 & \pm & 0.03 \\
\hline 38:1alkyl* & 0.14 & \pm & 0.02 & 0.12 & \pm & 0.02 \\
\hline $38: 2$ & 0.47 & \pm & 0.09 & 0.40 & \pm & 0.07 \\
\hline 38:2alkyl* & 0.33 & \pm & 0.03 & 0.28 & \pm & 0.04 \\
\hline $38: 3$ & 1.10 & \pm & 0.09 & 1.03 & \pm & 0.08 \\
\hline 38:3alkyl|** & 0.61 & \pm & 0.05 & 0.50 & \pm & 0.03 \\
\hline $38: 4$ & 1.97 & \pm & 0.20 & 2.00 & \pm & 0.22 \\
\hline 38:4alky|** & 1.12 & \pm & 0.04 & 1.00 & \pm & 0.06 \\
\hline $38: 5^{\star * *}$ & 1.62 & \pm & 0.13 & 2.00 & \pm & 0.14 \\
\hline 38:5alkyl & 1.06 & \pm & 0.08 & 1.10 & \pm & 0.04 \\
\hline $38: 6$ & 1.46 & \pm & 0.18 & 1.59 & \pm & 0.10 \\
\hline 38:6alkyl** & 0.71 & \pm & 0.06 & 0.58 & \pm & 0.05 \\
\hline 40:1alkyl & 0.09 & \pm & 0.01 & 0.10 & \pm & 0.02 \\
\hline 40:2alkyl & 0.06 & \pm & 0.00 & 0.06 & \pm & 0.01 \\
\hline 40:3alkyl** & 0.14 & \pm & 0.01 & 0.11 & \pm & 0.01 \\
\hline $40: 4$ & 0.23 & \pm & 0.02 & 0.22 & \pm & 0.02 \\
\hline 40:4alkyl & 0.17 & \pm & 0.01 & 0.17 & \pm & 0.01 \\
\hline $40: 5$ & 0.36 & \pm & 0.02 & 0.39 & \pm & 0.04 \\
\hline 40:5alkyl & 0.27 & \pm & 0.04 & 0.30 & \pm & 0.02 \\
\hline $40: 6$ & 0.84 & \pm & 0.07 & 0.90 & \pm & 0.12 \\
\hline 40:6alkyl** & 0.68 & \pm & 0.01 & 0.55 & \pm & 0.03 \\
\hline $40: 7$ & 0.82 & \pm & 0.06 & 0.88 & \pm & 0.08 \\
\hline 42:2alkyl & 0.05 & \pm & 0.01 & 0.06 & \pm & 0.01 \\
\hline 42:3alkyl* & 0.05 & \pm & 0.01 & 0.06 & \pm & 0.01 \\
\hline 42:5alkyl & 0.03 & \pm & 0.01 & 0.03 & \pm & 0.01 \\
\hline $42: 6$ & 0.07 & \pm & 0.01 & 0.07 & \pm & 0.01 \\
\hline 42:6alkyl| & 0.08 & \pm & 0.01 & 0.06 & \pm & 0.00 \\
\hline $42: 7$ & 0.13 & \pm & 0.02 & 0.14 & \pm & 0.01 \\
\hline $42: 8^{*}$ & 0.05 & \pm & 0.00 & 0.06 & \pm & 0.01 \\
\hline
\end{tabular}


Supplementary table 6. Molar percentages of phosphatidylethanolamine (PE) species in control and ANGPTL3 knock-down (shANGPTL3) IHH cells. Determined by ESI-MS/MS (mean \pm SD, n=6). Statistically significant differences between the groups are marked with asterisks; ${ }^{*} p<0.05,{ }^{* *} p<0.01,{ }^{* * *} p<0.001$ (twotailed Student's t-test).

\begin{tabular}{lrllrll} 
PE & \multicolumn{3}{c}{ control } & \multicolumn{3}{c}{ shANGPTL3 } \\
\hline $31: 0$ & 0.11 & \pm & 0.06 & 0.09 & \pm & 0.05 \\
$32: 0$ & 0.34 & \pm & 0.15 & 0.38 & \pm & 0.14 \\
$32: 1$ & 2.12 & \pm & 0.28 & 1.90 & \pm & 0.22 \\
$2: 2$ & 0.27 & \pm & 0.03 & 0.24 & \pm & 0.05 \\
$33: 1$ & 0.58 & \pm & 0.11 & 0.48 & \pm & 0.07 \\
$4: 1^{* *}$ & 11.38 & \pm & 0.77 & 9.79 & \pm & 0.46 \\
$34: 2^{* *}$ & 2.85 & \pm & 0.13 & 2.43 & \pm & 0.27 \\
$35: 1$ & 1.06 & \pm & 0.10 & 0.94 & \pm & 0.11 \\
$35: 2$ & 0.55 & \pm & 0.06 & 0.50 & \pm & 0.09 \\
$36: 1^{* * *}$ & 11.20 & \pm & 0.43 & 9.09 & \pm & 0.48 \\
$36: 2^{* *}$ & 13.67 & \pm & 0.69 & 12.34 & \pm & 0.23 \\
$36: 3$ & 1.83 & \pm & 0.19 & 1.72 & \pm & 0.20 \\
$36: 4$ & 2.71 & \pm & 0.25 & 2.62 & \pm & 0.33 \\
$36: 5^{* *}$ & 0.49 & \pm & 0.12 & 0.70 & \pm & 0.08 \\
$37: 4^{*}$ & 0.85 & \pm & 0.11 & 0.71 & \pm & 0.05 \\
$37: 5$ & 0.45 & \pm & 0.08 & 0.48 & \pm & 0.07 \\
$37: 6^{* *}$ & 0.53 & \pm & 0.07 & 0.36 & \pm & 0.10 \\
$38: 1$ & 0.38 & \pm & 0.08 & 0.41 & \pm & 0.08 \\
$38: 2$ & 1.22 & \pm & 0.18 & 1.16 & \pm & 0.16 \\
$38: 3$ & 3.55 & \pm & 0.22 & 3.97 & \pm & 0.44 \\
$38: 4$ & 14.85 & \pm & 0.76 & 15.36 & \pm & 0.83 \\
$38: 5^{* * *}$ & 8.18 & \pm & 0.26 & 11.36 & \pm & 0.16 \\
$38: 6^{* * *}$ & 2.67 & \pm & 0.21 & 3.53 & \pm & 0.26 \\
$39: 5$ & 0.35 & \pm & 0.05 & 0.36 & \pm & 0.06 \\
$39: 6^{*}$ & 0.68 & \pm & 0.06 & 0.60 & \pm & 0.04 \\
$40: 1$ & 0.33 & \pm & 0.07 & 0.34 & \pm & 0.04 \\
$40: 3$ & 0.40 & \pm & 0.06 & 0.47 & \pm & 0.08 \\
$40: 4$ & 0.82 & \pm & 0.17 & 0.96 & \pm & 0.10 \\
$40: 5^{*}$ & 1.73 & \pm & 0.23 & 2.09 & \pm & 0.31 \\
$40: 6$ & 6.76 & \pm & 0.36 & 6.95 & \pm & 0.41 \\
$40: 7^{*}$ & 4.49 & \pm & 0.34 & 4.98 & \pm & 0.30 \\
$40: 8$ & 0.47 & \pm & 0.12 & 0.48 & \pm & 0.15 \\
$42: 4$ & 0.21 & \pm & 0.03 & 0.20 & \pm & 0.03 \\
$42: 5$ & 0.19 & \pm & 0.04 & 0.23 & \pm & 0.06 \\
$42: 6$ & 0.23 & \pm & 0.03 & 0.27 & \pm & 0.04 \\
$42: 7$ & 0.33 & \pm & 0.05 & 0.35 & \pm & 0.08 \\
$42: 8$ & 0.18 & \pm & 0.06 & 0.18 & \pm & 0.04 \\
$42: 9$ & 0.26 & \pm & 0.08 & 0.25 & \pm & 0.05 \\
$42: 10$ & 0.15 & \pm & 0.02 & 0.17 & \pm & 0.05 \\
$43: 8$ & 0.37 & \pm & 0.06 & 0.37 & \pm & 0.07 \\
$43: 9$ & 0.22 & \pm & 0.05 & 0.19 & \pm & 0.04 \\
& & & & & &
\end{tabular}


Supplementary table 7. Molar percentages of phosphatidylinositol (PI) species in control and ANGPTL3 knock-down (shANGPTL3) IHH cells. Determined by ESI-MS/MS (mean $\pm S D, n=6$ ). Statistically significant differences between the groups are marked with asterisks; ${ }^{*} p<0.05,{ }^{* *} p<0.01,{ }^{* * *} p<0.001$ (two-tailed Student's t-test).

\begin{tabular}{lrllrll}
$\mathrm{PI}$ & \multicolumn{3}{c}{ control } & \multicolumn{3}{c}{ shANGPTL3 } \\
\hline $34: 01^{* * *}$ & 5.29 & \pm & 1.00 & 2.84 & \pm & 0.48 \\
$34: 02$ & 1.87 & \pm & 0.44 & 2.04 & \pm & 0.95 \\
$36: 00$ & 0.12 & \pm & 0.18 & 0.25 & \pm & 0.31 \\
$36: 01^{* * *}$ & 8.26 & \pm & 1.01 & 3.82 & \pm & 0.54 \\
$36: 02$ & 9.96 & \pm & 3.49 & 8.37 & \pm & 2.77 \\
$36: 03^{*}$ & 3.34 & \pm & 0.34 & 2.71 & \pm & 0.45 \\
$36: 04$ & 1.96 & \pm & 0.91 & 2.43 & \pm & 1.16 \\
$38: 02$ & 1.53 & \pm & 1.24 & 1.21 & \pm & 0.86 \\
$38: 03$ & 23.05 & \pm & 2.97 & 21.36 & \pm & 1.83 \\
$38: 04^{*}$ & 29.74 & \pm & 4.06 & 35.81 & \pm & 3.36 \\
$38: 05^{* * *}$ & 6.75 & \pm & 1.04 & 10.95 & \pm & 1.60 \\
$40: 03$ & 1.21 & \pm & 0.70 & 0.99 & \pm & 0.59 \\
$40: 04$ & 1.68 & \pm & 0.63 & 1.56 & \pm & 0.56 \\
$40: 05$ & 2.48 & \pm & 0.59 & 1.83 & \pm & 1.05 \\
$40: 06$ & 2.77 & \pm & 0.73 & 3.83 & \pm & 1.65
\end{tabular}

Supplementary table 8. Molar percentages of cholesterol ester (CE) species in control and ANGPTL3 knock-down (shANGPTL3) IHH cells. Determined by ESI-MS/MS (mean \pm SD, $n=6$ ). Statistically significant differences between the groups are marked with asterisks; ${ }^{*} p<0.05,{ }^{* *} p<0.01,{ }^{* * *} p<0.001$ (two-tailed Student's t-test).

\begin{tabular}{lrllrll} 
& \multicolumn{3}{c}{ control } & \multicolumn{3}{c}{ shANGPTL3 } \\
\hline $14: 0$ & 1.55 & \pm & 0.16 & 1.27 & \pm & 0.29 \\
$15: 0^{* * *}$ & 0.62 & \pm & 0.14 & 1.27 & \pm & 0.16 \\
$16: 0$ & 6.75 & \pm & 0.38 & 6.33 & \pm & 0.39 \\
$16: 1$ & 4.31 & \pm & 0.31 & 4.83 & \pm & 0.65 \\
$16: 2$ & 0.89 & \pm & 0.18 & 0.78 & \pm & 0.30 \\
$17: 0$ & 0.98 & \pm & 0.35 & 1.33 & \pm & 0.33 \\
$17: 1^{* *}$ & 1.45 & \pm & 0.16 & 1.84 & \pm & 0.25 \\
$18: 0$ & 0.09 & \pm & 0.12 & 0.13 & \pm & 0.31 \\
$18: 1^{* * *}$ & 31.03 & \pm & 1.08 & 23.26 & \pm & 1.63 \\
$18: 2$ & 4.42 & \pm & 0.40 & 4.68 & \pm & 0.39 \\
$18: 3$ & 0.83 & \pm & 0.13 & 1.04 & \pm & 0.25 \\
$19: 1$ & 1.03 & \pm & 0.22 & 0.89 & \pm & 0.20 \\
$19: 2$ & 0.64 & \pm & 0.10 & 0.74 & \pm & 0.29 \\
$20: 1^{* *}$ & 3.96 & \pm & 0.30 & 3.22 & \pm & 0.34 \\
$20: 2^{*}$ & 4.54 & \pm & 0.58 & 3.58 & \pm & 0.89 \\
$20: 3$ & 5.58 & \pm & 0.45 & 5.22 & \pm & 0.75 \\
$20: 4$ & 4.24 & \pm & 0.31 & 5.08 & \pm & 1.20 \\
$20: 5^{*}$ & 1.73 & \pm & 0.07 & 2.68 & \pm & 0.79 \\
$22: 1^{*}$ & 1.41 & \pm & 0.17 & 0.94 & \pm & 0.37 \\
$22: 2^{*}$ & 0.59 & \pm & 0.21 & 0.37 & \pm & 0.09 \\
$22: 3$ & 1.09 & \pm & 0.29 & 1.24 & \pm & 0.39 \\
$22: 4$ & 1.40 & \pm & 0.34 & 1.16 & \pm & 0.77 \\
$22: 5$ & 0.43 & \pm & 0.24 & 1.17 & \pm & 1.15 \\
$22: 6^{* * *}$ & 12.17 & \pm & 0.70 & 21.24 & \pm & 2.01 \\
$24: 1$ & 1.99 & \pm & 0.53 & 1.44 & \pm & 0.31 \\
$24: 5$ & 0.73 & \pm & 0.49 & 0.30 & \pm & 0.20 \\
$24: 6^{* *}$ & 3.79 & \pm & 0.48 & 2.90 & \pm & 0.30 \\
$26: 6^{* * *}$ & 1.77 & \pm & 0.13 & 1.04 & \pm & 0.31
\end{tabular}


Supplementary table 9. Lipid mediators (pg/well) in control and ANGPTL3 knock-down cells (shANGPTL3), and transitions used for determining the species by LC-MS/MS Data represent mean $\pm \mathrm{SD}(n=3)$

\begin{tabular}{|c|c|c|c|c|c|c|c|c|}
\hline \multirow{3}{*}{ DHA Bioactive metabolome } & \multicolumn{2}{|c|}{ Transition } & \multirow{2}{*}{\multicolumn{3}{|c|}{ control }} & \multirow{2}{*}{\multicolumn{3}{|c|}{ shANGPTL3 }} \\
\hline & \multirow{2}{*}{ Q1 } & \multirow[t]{2}{*}{ Q3 } & & & & & & \\
\hline & & & & & & & & \\
\hline RvD5 & 359 & 199 & 0.56 & \pm & 0.97 & 3.02 & \pm & 3.13 \\
\hline RvD6 & 359 & 159 & 0.26 & \pm & 0.45 & 3.50 & \pm & 2.99 \\
\hline 17R-RvD3 & 375 & 147 & 0.44 & \pm & 0.42 & 0.78 & \pm & 0.67 \\
\hline 10S,17S-diHDHA & 359 & 153 & 2.67 & \pm & 3.95 & 14.63 & \pm & 8.83 \\
\hline MaR2 & 359 & 221 & 0.00 & \pm & 0.00 & 2.43 & \pm & 3.80 \\
\hline 22-OH-MaR1 & 375 & 221 & 13.26 & \pm & 22.97 & 37.89 & \pm & 58.43 \\
\hline 7S,14S-diHDHA & 359 & 177 & 0.57 & \pm & 0.94 & 1.35 & \pm & 2.33 \\
\hline 4,14-diHDHA & 359 & 159 & 0.57 & \pm & 0.76 & 1.99 & \pm & 2.96 \\
\hline \multicolumn{9}{|c|}{ n-3 DPA bioactive metabolome } \\
\hline RvT1 & 377 & 193 & 0.74 & \pm & 0.31 & 1.24 & \pm & 0.36 \\
\hline RvT4 & 361 & 211 & 1.39 & \pm & 0.53 & 4.29 & \pm & 4.07 \\
\hline RvD1 ${ }_{n-3}$ DPA & 377 & 143 & 1.36 & \pm & 0.86 & 6.52 & \pm & 10.85 \\
\hline RvD2 $2_{n-3 D P A}$ & 377 & 233 & 0.00 & \pm & 0.00 & 0.41 & \pm & 0.71 \\
\hline $\operatorname{RvD5}_{n-3 D P A}$ & 361 & 143 & 1.17 & \pm & 2.03 & 3.02 & \pm & 3.46 \\
\hline 10S, 17S-diHDPA & 361 & 183 & 0.94 & \pm & 0.86 & 4.03 & \pm & 2.80 \\
\hline 22-OH-PD1 $1_{n-3}$ DPA & 377 & 183 & 0.00 & \pm & 0.00 & 0.11 & \pm & 0.19 \\
\hline$M a R 2_{n-3}$ DPA & 361 & 193 & 1.05 & \pm & 1.14 & 2.96 & \pm & 3.99 \\
\hline \multicolumn{9}{|l|}{ EPA bioative metabolome } \\
\hline RvE2 & 333 & 159 & 9.18 & \pm & 12.08 & 2.40 & \pm & 0.75 \\
\hline \multicolumn{9}{|l|}{ AA bioative metabolome } \\
\hline $\begin{array}{l}\text { 5S,15S-diHETE } \\
\text { 15-epi-LXA } \\
\text { 13,14-dehydro-15-oxo-LXA }\end{array}$ & $\begin{array}{l}335 \\
351 \\
351\end{array}$ & $\begin{array}{l}115 \\
115 \\
217\end{array}$ & $\begin{array}{l}1.26 \\
1.95 \\
0.00\end{array}$ & $\begin{array}{l} \pm \\
\pm \\
\pm\end{array}$ & $\begin{array}{l}0.66 \\
2.89 \\
0.00\end{array}$ & $\begin{array}{r}5.25 \\
19.68 \\
4.52\end{array}$ & $\begin{array}{l} \pm \\
\pm \\
\pm\end{array}$ & $\begin{array}{l}5.63 \\
30.04 \\
7.83\end{array}$ \\
\hline $\begin{array}{l}\mathrm{LTB}_{4} \\
\text { 5S,12S-diHETE } \\
\text { 6-trans-LTB } 4\end{array}$ & $\begin{array}{l}335 \\
335 \\
335\end{array}$ & $\begin{array}{l}195 \\
195 \\
195\end{array}$ & $\begin{array}{l}0.69 \\
1.50 \\
3.18\end{array}$ & $\begin{array}{l} \pm \\
\pm \\
\pm\end{array}$ & $\begin{array}{l}1.01 \\
2.59 \\
2.97\end{array}$ & $\begin{array}{r}9.76 \\
16.10 \\
5.17\end{array}$ & $\begin{array}{l} \pm \\
\pm \\
\pm\end{array}$ & $\begin{array}{l}13.27 \\
23.92 \\
3.54\end{array}$ \\
\hline
\end{tabular}


6-trans-12-epi LTB 4

$\mathrm{PGD}_{2}$

$\mathrm{PGE}_{2}$

$\mathrm{PGF}_{2 a}$

$\mathrm{TxB}_{2}$

Sum RvD

Sum PD

Sum MaR

Sum RvT

Sum $R_{V} D_{n-3}$ DPA

Sum $P D_{n-3}$ DPA

Sum $M_{a R} R_{n-3} D P A$

Sum RvE

Sum LX

Sum $\mathrm{LTB}_{4}$ met

Sum PG

Sum TX

$\begin{array}{ll}335 & 195 \\ 351 & 189 \\ 351 & 189 \\ 353 & 193 \\ 369 & 169\end{array}$

$2.55 \pm 2.36$

$0.25 \pm 0.43$

$1.40 \pm 1.78$

$0.53 \pm 0.84$

$2.17 \pm 3.76$

$5.07 \pm 5.66$

$5.03 \pm 4.18$

$9.50 \pm 11.78$

$6.19 \pm 7.70$

$21.62 \pm 32.81$

\section{control}

$1.26 \pm 1.83$

$2.67 \pm 3.95$

$14.40 \pm 24.66$

$2.13 \pm 0.81$

$2.53 \pm 2.86$

$0.94 \pm 0.86$

$1.05 \pm 1.14$

$9.18 \pm 12.08$

$3.20 \pm 3.54$

$7.92 \pm 8.90$

$2.18 \pm 3.03$

$2.17 \pm 3.76$

\section{ShANGPTL3}

$7.29 \pm 5.44$

$14.63 \pm 8.83$

$41.22 \pm 63.70$

$5.53 \pm 4.37$

$9.96 \pm 13.84$

$4.14 \pm 2.75$

$2.96 \pm 3.99$

$2.40 \pm 0.75$

$29.45 \pm 43.49$

$36.11 \pm 46.38$

$20.72 \pm 23.60$

$21.62 \pm 32.81$ 


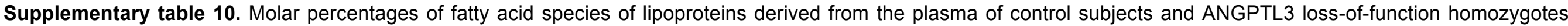

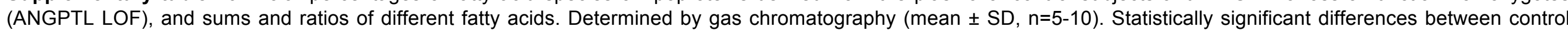

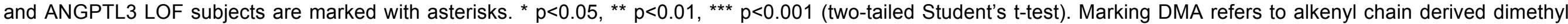
acetal, SAT=saturated, MUFA=monounsaturated, PUFA=polyunsaturated, UNSAT=unsaturated.

VLDL

LDL

HDL

\begin{tabular}{|c|c|c|c|c|c|c|c|c|c|c|c|c|c|c|c|c|c|c|c|c|}
\hline FA & \multicolumn{3}{|c|}{ control } & \multicolumn{3}{|c|}{ ANGPTL3 LOF } & \multirow{2}{*}{$\begin{array}{l}\text { FA } \\
14: 0\end{array}$} & \multicolumn{3}{|c|}{ control } & \multicolumn{3}{|c|}{ ANGPTL3 LOF } & \multirow{2}{*}{$\begin{array}{l}\text { FA } \\
14: 0\end{array}$} & \multicolumn{3}{|c|}{ control } & \multicolumn{3}{|c|}{ ANGPTL3 LOF } \\
\hline 14:0 & 1.38 & \pm & 0.71 & 1.88 & \pm & 0.43 & & 0.89 & \pm & 0.32 & 1.30 & \pm & 0.72 & & 0.66 & \pm & 0.23 & 1.61 & \pm & 1.80 \\
\hline $15: 0^{* *}$ & 0.25 & \pm & 0.10 & 0.53 & \pm & 0.15 & $15: 0$ & 0.21 & \pm & 0.07 & 0.25 & \pm & 0.04 & $15: 0^{*}$ & 0.18 & \pm & 0.06 & 0.24 & \pm & 0.04 \\
\hline DMA16:0 ** & 0.22 & \pm & 0.07 & 0.33 & \pm & 0.06 & DMA16:0 & 0.34 & \pm & 0.10 & 0.32 & \pm & 0.12 & DMA16:0 & 0.54 & \pm & 0.15 & 0.69 & \pm & 0.15 \\
\hline $16: 0$ & 25.86 & \pm & 2.12 & 27.40 & \pm & 0.64 & $16: 0$ & 23.06 & \pm & 1.56 & 24.33 & \pm & 0.91 & $16: 0$ & 24.16 & \pm & 1.66 & 24.09 & \pm & 1.24 \\
\hline $16: 1 n-9^{* * *}$ & 0.63 & \pm & 0.08 & 1.38 & \pm & 0.15 & $16: 1 n-9^{* *}$ & 0.44 & \pm & 0.07 & 0.80 & \pm & 0.20 & $16: 1 n-9^{*}$ & 0.34 & \pm & 0.05 & 0.66 & \pm & 0.21 \\
\hline $16: 1 n-7$ & 2.48 & \pm & 0.60 & 3.52 & \pm & 1.66 & $16: 1 n-7$ & 2.01 & \pm & 0.56 & 3.68 & \pm & 1.79 & $16: 1 n-7$ & 1.56 & \pm & 0.41 & 3.15 & \pm & 1.71 \\
\hline $17: 0^{*}$ & 0.23 & \pm & 0.05 & 0.39 & \pm & 0.11 & $17: 0$ & 0.20 & \pm & 0.05 & 0.22 & \pm & 0.07 & $17: 0$ & 0.20 & \pm & 0.04 & 0.22 & \pm & 0.05 \\
\hline $17: 1 n-8$ & 0.16 & \pm & 0.05 & 0.21 & \pm & 0.09 & $17: 1 n-8$ & 0.13 & \pm & 0.04 & 0.21 & \pm & 0.08 & $17: 1 n-8$ & 0.10 & \pm & 0.03 & 0.18 & \pm & 0.09 \\
\hline DMA18:0 & 0.11 & \pm & 0.03 & 0.12 & \pm & 0.07 & DMA18:0 & 0.18 & \pm & 0.05 & 0.13 & \pm & 0.08 & DMA18:0 & 0.27 & \pm & 0.07 & 0.30 & \pm & 0.13 \\
\hline DMA18:1n-9* & 0.07 & \pm & 0.05 & 0.28 & \pm & 0.17 & DMA18:1n-9 & 0.07 & \pm & 0.02 & 0.08 & \pm & 0.03 & DMA18:1n-9** & 0.14 & \pm & 0.03 & 0.23 & \pm & 0.05 \\
\hline $18: 0^{* *}$ & 5.69 & \pm & 0.56 & 10.32 & \pm & 2.39 & $18: 0^{\star * *}$ & 5.78 & \pm & 0.40 & 5.19 & \pm & 0.09 & $18: 0^{* * *}$ & 7.26 & \pm & 0.52 & 6.02 & \pm & 0.25 \\
\hline $18: 1 n-9^{*}$ & 29.89 & \pm & 3.79 & 25.41 & \pm & 2.95 & $18: 1 n-9^{*}$ & 22.53 & \pm & 2.49 & 26.24 & \pm & 2.16 & $18: 1 n-9$ & 18.40 & \pm & 1.89 & 21.66 & \pm & 2.78 \\
\hline $18: 1 n-7$ & 2.22 & \pm & 0.30 & 2.20 & \pm & 0.24 & $18: 1 n-7^{* *}$ & 1.71 & \pm & 0.22 & 2.06 & \pm & 0.20 & $18: 1 n-7$ & 1.57 & \pm & 0.22 & 1.76 & \pm & 0.23 \\
\hline $18: 2 n-6^{* * *}$ & 21.27 & \pm & 3.41 & 13.43 & \pm & 2.35 & $18: 2 n-6^{* *}$ & 28.97 & \pm & 3.71 & 20.15 & \pm & 3.55 & $18: 2 n-6^{*}$ & 27.95 & \pm & 3.88 & 21.09 & \pm & 4.24 \\
\hline $18: 3 n-6^{*}$ & 0.34 & \pm & 0.10 & 0.69 & \pm & 0.27 & $18: 3 n-6$ & 0.46 & \pm & 0.14 & 0.88 & \pm & 0.44 & $18: 3 n-6$ & 0.36 & \pm & 0.11 & 0.76 & \pm & 0.44 \\
\hline $18: 3 n-3$ & 0.38 & \pm & 0.09 & 0.28 & \pm & 0.12 & $18: 3 n-3$ & 0.28 & \pm & 0.05 & 0.25 & \pm & 0.08 & $18: 3 n-3$ & 0.22 & \pm & 0.04 & 0.21 & \pm & 0.05 \\
\hline $20: 0^{\star *}$ & 0.19 & \pm & 0.04 & 0.40 & \pm & 0.08 & $20: 0^{\star *}$ & 0.29 & \pm & 0.05 & 0.43 & \pm & 0.07 & $20: 0^{* *}$ & 0.24 & \pm & 0.04 & 0.39 & \pm & 0.07 \\
\hline $20: 1 n-9$ & 0.25 & \pm & 0.07 & 0.65 & \pm & 0.46 & $20: 1 n-9$ & 0.13 & \pm & 0.02 & 0.17 & \pm & 0.05 & $20: 1 n-9$ & 0.12 & \pm & 0.02 & 0.13 & \pm & 0.04 \\
\hline $20: 2 n-6$ & 0.17 & \pm & 0.05 & 0.24 & \pm & 0.16 & $20: 2 n-6$ & 0.14 & \pm & 0.03 & 0.14 & \pm & 0.04 & $20: 2 n-6$ & 0.16 & \pm & 0.04 & 0.15 & \pm & 0.04 \\
\hline $20: 3 n-9^{*}$ & 0.22 & \pm & 0.11 & 0.77 & \pm & 0.40 & $20: 3 n-9$ & 0.17 & \pm & 0.06 & 0.52 & \pm & 0.30 & $20: 3 n-9$ & 0.18 & \pm & 0.07 & 0.47 & \pm & 0.31 \\
\hline $20: 3 n-6$ & 0.93 & \pm & 0.17 & 1.00 & \pm & 0.13 & $20: 3 n-6$ & 1.34 & \pm & 0.27 & 1.26 & \pm & 0.17 & $20: 3 n-6$ & 1.88 & \pm & 0.38 & 1.79 & \pm & 0.25 \\
\hline
\end{tabular}




\begin{tabular}{|c|c|c|c|c|c|c|c|c|c|c|c|c|c|c|c|c|c|c|c|c|}
\hline $20: 4 n-6$ & 4.24 & \pm & 1.22 & 4.60 & \pm 1 & 1.02 & $20: 4 n-6$ & 6.52 & \pm & 1.63 & 6.16 & \pm & 1.06 & $20: 4 n-6$ & 8.74 & \pm & 2.08 & 8.23 & \pm & 1.17 \\
\hline $20: 5 n-3$ & 0.32 & \pm & 0.15 & 0.44 & $\pm c$ & 0.10 & $20: 5 n-3$ & 0.47 & \pm & 0.19 & 0.52 & \pm & 0.17 & $20: 5 n-3$ & 0.60 & \pm & 0.26 & 0.59 & \pm & 0.17 \\
\hline $22: 0^{\star \star \star}$ & 0.33 & \pm & 0.09 & 0.49 & $\pm c$ & 0.03 & $22: 0^{* *}$ & 0.58 & \pm & 0.11 & 0.80 & $\pm c$ & 0.13 & $22: 0^{* *}$ & 0.47 & \pm & 0.07 & 0.78 & \pm & 0.13 \\
\hline $23: 0^{* * *}$ & 0.16 & \pm & 0.02 & 0.24 & $\pm c$ & 0.01 & $23: 0^{*}$ & 0.28 & \pm & 0.04 & 0.37 & $\pm c$ & 0.07 & $23: 0^{\star *}$ & 0.23 & \pm & 0.04 & 0.36 & \pm & 0.06 \\
\hline $22: 5 n-3$ & 0.21 & \pm & 0.06 & 0.26 & $\pm c$ & 0.06 & $22: 5 n-3$ & 0.21 & \pm & 0.05 & 0.22 & $\pm c$ & 0.06 & $22: 5 n-3$ & 0.32 & \pm & 0.07 & 0.29 & \pm & 0.08 \\
\hline $24: 0^{* *}$ & 0.27 & \pm & 0.07 & 0.43 & $\pm c$ & 0.07 & $24: 0$ & 0.45 & \pm & 0.09 & 0.54 & $\pm c$ & 0.11 & $24: 0^{*}$ & 0.35 & \pm & 0.06 & 0.56 & \pm & 0.14 \\
\hline $22: 6 n-3$ & 0.93 & \pm & 0.22 & 0.99 & $\pm c$ & 0.24 & $22: 6 n-3$ & 1.17 & \pm & 0.22 & 1.02 & \pm & 0.16 & $22: 6 n-3^{*}$ & 1.87 & \pm & 0.35 & 1.51 & \pm & 0.18 \\
\hline $24: 1 n-9^{* * *}$ & 0.61 & \pm & 0.14 & 1.11 & $\pm c$ & 0.15 & $24: 1 n-9^{* *}$ & 1.00 & \pm & 0.15 & 1.76 & $\pm c$ & 0.29 & $24: 1 n-9^{* *}$ & 0.93 & \pm & 0.16 & 1.88 & \pm & 0.43 \\
\hline SUM SAT** & 34.36 & \pm & 2.62 & 42.08 & \pm 3 & 3.47 & SUM SAT & 31.73 & \pm & 1.43 & 33.44 & \pm & 1.50 & SUM SAT & 33.75 & \pm & 1.46 & 34.25 & \pm & 2.37 \\
\hline SUM MUFA & 36.30 & \pm & 4.18 & 34.77 & \pm 4 & 4.53 & SUM MUFA* & 28.02 & \pm & 2.84 & 35.00 & \pm & 4.21 & SUM MUFA* & 23.17 & \pm & 2.19 & 29.65 & \pm & 5.01 \\
\hline SUM PUFA** & 29.00 & \pm & 4.00 & 22.70 & \pm 2 & 2.26 & SUM PUFA $A^{\star \star \star}$ & 39.72 & \pm & 3.26 & 31.12 & \pm 2 & 2.96 & SUM PUFA & 42.27 & \pm & 2.95 & 35.10 & \pm & 3.54 \\
\hline SUM n-6*** & 26.94 & \pm & 3.86 & 19.97 & \pm 2 & 2.22 & SUM n- $6^{* * *}$ & 37.43 & \pm & 3.32 & 28.59 & \pm 3 & 3.26 & SUM n- $6^{* *}$ & 39.09 & \pm & 3.13 & 32.03 & \pm & 3.93 \\
\hline SUM n-3 & 1.85 & \pm & 0.34 & 1.97 & $\pm c$ & 0.41 & SUM n-3 & 2.13 & \pm & 0.38 & 2.00 & $\pm c$ & 0.22 & SUM n-3 & 3.01 & \pm & 0.57 & 2.60 & \pm & 0.25 \\
\hline UNSAT/SAT $T^{* * *}$ & 1.92 & \pm & 0.22 & 1.38 & $\pm c$ & 0.21 & UNSAT/SAT & 2.14 & \pm & 0.14 & 1.98 & $\pm c$ & 0.13 & UNSAT/SAT & 1.94 & \pm & 0.13 & 1.90 & \pm & 0.18 \\
\hline$n-3 / n-6^{*}$ & 0.07 & \pm & 0.01 & 0.10 & $\pm c$ & 0.02 & $n-3 / n-6$ & 0.06 & \pm & 0.01 & 0.07 & $\pm c$ & 0.01 & $n-3 / n-6$ & 0.08 & \pm & 0.02 & 0.08 & \pm & 0.02 \\
\hline $\mathrm{DMA}^{*}$ & 0.40 & \pm & 0.12 & 0.73 & \pm 0 & 0.23 & DMA & 0.59 & \pm & 0.17 & 0.52 & \pm & 0.22 & DMA & 0.95 & \pm & 0.25 & 1.23 & \pm & 0.32 \\
\hline
\end{tabular}


Supplementary table 11. Fold changes of total lipid concentrations (pmol/ml plasma) in lipoproteins of ANGPTL3 lossof-function homozygotes compared to control subjects. The average concentration in control subjects was set to 1 (mean $\pm \mathrm{SD}, \mathrm{n}=4-10)$. Statistically significant differences between the groups are marked with asterisks; ${ }^{* *} \mathrm{p}<0.01 .{ }^{* * *}$ p $<0.001$ (two-tailed Student's t-test).

\begin{tabular}{lclc} 
Lipid & VLDL & \multicolumn{1}{c}{ LDL } & HDL \\
\hline TAG & $0.070 \pm 0.045^{* * *}$ & $1.049 \pm 0.340$ & $0.531 \pm 0.066^{* *}$ \\
CE & $0.078 \pm 0.045^{* * *}$ & $0.436 \pm 0.076^{* * *}$ & $0.379 \pm 0.073^{* * *}$ \\
LysoPC & $0.104 \pm 0.059^{* * *}$ & $0.500 \pm 0.182^{* *}$ & $0.316 \pm 0.105^{* * *}$ \\
PC & $0.086 \pm 0.053^{* * *}$ & $0.452 \pm 0.052^{* * *}$ & $0.281 \pm 0.051^{* * *}$ \\
SM & $0.157 \pm 0.098^{* * *}$ & $0.905 \pm 0.181$ & $0.569 \pm 0.089^{* * *}$
\end{tabular}


Supplementary table 12. Molar percentages of triacylglycerol (TAG) species in lipoproteins of control subjects and ANGPTL3 loss-of-function homozygotes (ANGPTL3 LOF). Determined by ESI-MS/MS (mean $\pm \mathrm{SD}, \mathrm{n}=4-10$ ). Statistically significant differences between the groups are marked with asterisks; ${ }^{*} p<0.05,{ }^{* \star} p<0.01,{ }^{* * *} p<0.001$ (two-tailed Student's t-test).

VLDL

\begin{tabular}{|c|c|c|c|c|c|c|}
\hline TAG & \multicolumn{3}{|c|}{ control } & \multicolumn{3}{|c|}{ ANGPTL3 LOF } \\
\hline $48: 0$ & 0.67 & \pm & 0.36 & 0.53 & \pm & 0.08 \\
\hline $48: 1$ & 2.37 & \pm & 1.28 & 2.22 & \pm & 0.82 \\
\hline $48: 2$ & 1.81 & \pm & 0.89 & 1.96 & \pm & 0.83 \\
\hline $48: 3$ & 0.58 & \pm & 0.29 & 0.46 & \pm & 0.29 \\
\hline 49:4 & 0.22 & \pm & 0.03 & 0.20 & \pm & 0.21 \\
\hline $50: 0$ & 0.28 & \pm & 0.32 & 0.07 & \pm & 0.13 \\
\hline $50: 1$ & 6.33 & \pm & 1.73 & 5.34 & \pm & 1.29 \\
\hline $50: 2$ & 8.28 & \pm & 1.70 & 9.79 & \pm & 2.14 \\
\hline $50: 3$ & 3.23 & \pm & 0.63 & 3.03 & \pm & 0.64 \\
\hline $50: 4$ & 0.70 & \pm & 0.22 & 0.58 & \pm & 0.41 \\
\hline $50: 6$ & 0.27 & \pm & 0.07 & 0.21 & \pm & 0.29 \\
\hline $51: 2$ & 0.95 & \pm & 0.32 & 0.80 & \pm & 0.32 \\
\hline 51:3 & 0.49 & \pm & 0.18 & 0.26 & \pm & 0.31 \\
\hline $52: 0$ & 0.13 & \pm & 0.10 & 0.16 & \pm & 0.07 \\
\hline $52: 1$ & 1.34 & \pm & 0.72 & 1.10 & \pm & 0.74 \\
\hline $52: 2$ & 24.48 & \pm & 4.55 & 23.86 & \pm & 1.78 \\
\hline $52: 3$ & 18.29 & \pm & 2.47 & 15.71 & \pm & 4.03 \\
\hline $52: 4$ & 5.88 & \pm & 1.60 & 5.02 & \pm & 1.00 \\
\hline $52: 5$ & 1.18 & \pm & 0.32 & 1.33 & \pm & 0.34 \\
\hline $52: 6$ & 0.25 & \pm & 0.10 & 0.27 & \pm & 0.25 \\
\hline $52: 8$ & 0.38 & \pm & 0.21 & 0.23 & \pm & 0.21 \\
\hline $53: 2$ & 0.56 & \pm & 0.14 & 0.45 & \pm & 0.06 \\
\hline $53: 3^{\star *}$ & 0.51 & \pm & 0.08 & 0.25 & \pm & 0.09 \\
\hline $54: 2$ & 1.55 & \pm & 0.24 & 1.77 & \pm & 0.36 \\
\hline $54: 3$ & 6.16 & \pm & 1.59 & 6.45 & \pm & 1.21 \\
\hline $54: 4$ & 4.82 & \pm & 1.24 & 5.00 & \pm & 1.61 \\
\hline $54: 5^{* *}$ & 2.82 & \pm & 0.86 & 4.06 & \pm & 0.59 \\
\hline $54: 6^{*}$ & 1.23 & \pm & 0.42 & 1.91 & \pm & 0.40 \\
\hline
\end{tabular}

LDL

\begin{tabular}{|c|c|c|c|c|}
\hline TAG & \multicolumn{2}{|c|}{ control } & \multicolumn{2}{|c|}{ ANGPTL3 LOF } \\
\hline 48:0 & 1.06 & \pm 0.32 & 0.76 & \pm 0.21 \\
\hline $48: 1$ & 2.61 & \pm 1.29 & 2.11 & \pm 0.51 \\
\hline $48: 2$ & 2.03 & \pm 0.72 & 1.55 & \pm 0.40 \\
\hline $48: 3$ & 0.96 & \pm 0.19 & 0.77 & \pm 0.16 \\
\hline $49: 4$ & 0.80 & \pm 0.19 & 0.65 & \pm 0.23 \\
\hline 50:0 & 0.42 & \pm 0.13 & 0.45 & \pm 0.30 \\
\hline $50: 1$ & 5.99 & \pm 2.25 & 5.56 & \pm 0.97 \\
\hline $50: 2$ & 7.61 & \pm 1.74 & 7.68 & \pm 1.61 \\
\hline $50: 3$ & 2.89 & \pm 0.42 & 2.63 & \pm 0.29 \\
\hline $50: 4$ & 0.70 & \pm 0.15 & 0.76 & \pm 0.17 \\
\hline $50: 6$ & 0.52 & \pm 0.11 & 0.45 & \pm 0.09 \\
\hline $51: 2$ & 1.00 & \pm 0.26 & 1.11 & \pm 0.24 \\
\hline $51: 3$ & 0.64 & \pm 0.15 & 0.64 & \pm 0.21 \\
\hline 52:0 & 0.30 & \pm 0.10 & 0.32 & \pm 0.06 \\
\hline $52: 1$ & 1.37 & \pm 0.41 & 1.41 & \pm 0.45 \\
\hline $52: 2$ & 23.32 & \pm 3.56 & 22.27 & \pm 2.40 \\
\hline $52: 3$ & 16.55 & \pm 2.30 & 14.07 & \pm 3.07 \\
\hline $52: 4$ & 5.27 & \pm 1.41 & 4.61 & \pm 0.94 \\
\hline $52: 5$ & 1.22 & \pm 0.33 & 1.56 & \pm 0.37 \\
\hline $52: 6$ & 0.45 & \pm 0.27 & 0.59 & \pm 0.23 \\
\hline $52: 8$ & 0.47 & \pm 0.14 & 0.45 & \pm 0.12 \\
\hline $53: 2$ & 0.66 & \pm 0.18 & 0.72 & \pm 0.20 \\
\hline $53: 3$ & 0.61 & \pm 0.11 & 0.71 & \pm 0.12 \\
\hline $54: 2$ & 1.79 & \pm 0.26 & 1.94 & \pm 0.25 \\
\hline $54: 3$ & 6.47 & \pm 1.40 & 6.37 & \pm 1.29 \\
\hline $54: 4$ & 4.93 & \pm 1.40 & 4.86 & \pm 1.21 \\
\hline $54: 5^{* *}$ & 2.98 & \pm 0.82 & 4.16 & \pm 0.56 \\
\hline $54: 6^{* *}$ & 1.33 & \pm 0.38 & 2.22 & \pm 0.41 \\
\hline
\end{tabular}

HDL

\begin{tabular}{|c|c|c|c|c|c|c|}
\hline TAG & \multicolumn{3}{|c|}{ control } & \multicolumn{3}{|c|}{ ANGPTL3 LOF } \\
\hline $48: 0$ & 2.00 & \pm & 0.24 & 1.52 & \pm & 0.42 \\
\hline $48: 1$ & 3.75 & \pm & 1.09 & 2.97 & \pm & 0.64 \\
\hline $48: 2^{*}$ & 3.52 & \pm & 0.66 & 2.52 & \pm & 0.62 \\
\hline $48: 3$ & 2.20 & \pm & 0.37 & 1.93 & \pm & 0.29 \\
\hline $49: 4^{*}$ & 2.16 & \pm & 0.51 & 1.54 & \pm & 0.40 \\
\hline $50: 0$ & 0.83 & \pm & 0.18 & 0.83 & \pm & 0.35 \\
\hline $50: 1$ & 5.95 & \pm & 1.85 & 5.31 & \pm & 1.06 \\
\hline $50: 2$ & 7.32 & \pm & 1.65 & 7.21 & \pm & 1.65 \\
\hline $50: 3$ & 2.99 & \pm & 0.35 & 2.67 & \pm & 0.37 \\
\hline $50: 4$ & 0.89 & \pm & 0.13 & 0.80 & \pm & 0.15 \\
\hline $50: 6^{*}$ & 1.17 & \pm & 0.26 & 0.90 & \pm & 0.15 \\
\hline $51: 2$ & 1.21 & \pm & 0.22 & 1.17 & \pm & 0.23 \\
\hline $51: 3$ & 0.88 & \pm & 0.17 & 0.83 & \pm & 0.29 \\
\hline $52: 0$ & 0.59 & \pm & 0.18 & 0.50 & \pm & 0.23 \\
\hline $52: 1$ & 1.78 & \pm & 0.42 & 1.90 & \pm & 0.61 \\
\hline $52: 2$ & 19.95 & \pm & 3.03 & 21.53 & \pm & 2.40 \\
\hline $52: 3$ & 14.43 & \pm & 1.74 & 12.18 & \pm & 2.98 \\
\hline $52: 4$ & 4.54 & \pm & 1.13 & 4.12 & \pm & 0.87 \\
\hline $52: 5$ & 1.04 & \pm & 0.29 & 1.33 & \pm & 0.26 \\
\hline $52: 6$ & 0.54 & \pm & 0.12 & 0.72 & \pm & 0.29 \\
\hline $52: 8$ & 0.73 & \pm & 0.17 & 0.60 & \pm & 0.19 \\
\hline $53: 2$ & 0.74 & \pm & 0.11 & 0.79 & \pm & 0.28 \\
\hline $53: 3$ & 0.60 & \pm & 0.12 & 0.59 & \pm & 0.14 \\
\hline $54: 2$ & 2.15 & \pm & 0.23 & 1.93 & \pm & 0.26 \\
\hline $54: 3$ & 5.81 & \pm & 1.57 & 5.85 & \pm & 1.12 \\
\hline $54: 4$ & 4.35 & \pm & 1.23 & 4.37 & \pm & 0.89 \\
\hline $54: 5^{* *}$ & 2.68 & \pm & 0.66 & 3.88 & \pm & 0.55 \\
\hline $54: 6^{\star \star}$ & 1.21 & \pm & 0.28 & 2.09 & \pm & 0.38 \\
\hline
\end{tabular}




\begin{tabular}{|c|c|c|c|c|c|c|c|c|c|c|c|c|c|c|c|c|}
\hline $54: 7$ & 0.33 & \pm & 0.10 & 0.36 & \pm 0.30 & $54: 7^{*}$ & 0.37 & \pm 0.09 & 0.68 & \pm 0.17 & $54: 7^{*}$ & 0.33 & \pm & 0.08 & 0.60 & \pm 0.18 \\
\hline $56: 5$ & 0.80 & \pm & 0.24 & 1.37 & \pm 0.42 & $56: 5^{*}$ & 1.04 & \pm 0.24 & 1.62 & 0.38 & $56: 5^{*}$ & 0.88 & \pm & 0.22 & 1.56 & 0.38 \\
\hline $56: 6^{*}$ & 1.22 & \pm & 0.29 & 2.35 & \pm 0.56 & $56: 6^{* *}$ & 1.44 & \pm 0.29 & 2.48 & 0.46 & $56: 6^{* *}$ & 1.15 & \pm & 0.29 & 2.09 & 0.42 \\
\hline $56: 7^{* *}$ & 1.13 & \pm & 0.34 & 1.78 & \pm 0.21 & $56: 7^{\star \star \star}$ & 1.26 & \pm 0.36 & 1.98 & 0.18 & $56: 7^{\star * *}$ & 0.92 & \pm & 0.20 & 1.71 & 0.20 \\
\hline $56: 8$ & 0.45 & \pm & 0.18 & 0.69 & \pm 0.31 & $56: 8^{\star * \star}$ & 0.54 & \pm 0.24 & 1.02 & \pm 0.06 & $56: 8^{* *}$ & 0.43 & \pm & 0.14 & 0.82 & 0.12 \\
\hline $58: 8$ & 0.30 & \pm & 0.09 & 0.43 & \pm 0.21 & $58: 8^{* * *}$ & 0.41 & \pm 0.26 & 0.84 & \pm 0.07 & $58: 8^{* * *}$ & 0.29 & \pm & 0.08 & 0.63 & 0.07 \\
\hline
\end{tabular}

Supplementary table 13. Molar percentages of cholesterol ester (CE) species in lipoproteins of control subjects and ANGPTL3 loss-of-function homozygotes (ANGPTL3 LOF). Determined by ESI-MS/MS (mean \pm SD, $n=5-10$ ). Statistically significant differences between the groups are marked with asterisks; * $p<0.05,{ }^{* *} p<0.01$ (two-tailed Student's t-test).

VLDL

\begin{tabular}{|c|c|c|c|c|c|}
\hline CE & \multicolumn{3}{|c|}{ control } & \multicolumn{2}{|c|}{ ANGPTL3 LOF } \\
\hline $16: 0^{*}$ & 6.04 & \pm & 0.62 & 7.63 & \pm 0.98 \\
\hline $16: 1$ & 2.98 & \pm & 0.97 & 6.06 & \pm 3.18 \\
\hline 18:1 & 13.37 & \pm & 1.64 & 17.84 & \pm 3.16 \\
\hline $18: 2^{*}$ & 59.91 & \pm & 3.35 & 49.71 & \pm 9.43 \\
\hline $18: 3$ & 2.75 & \pm & 0.49 & 3.81 & \pm 1.93 \\
\hline $20: 4$ & 12.33 & \pm & 3.21 & 12.99 & \pm 1.64 \\
\hline $20: 5$ & 1.40 & \pm & 0.58 & 1.15 & \pm 0.46 \\
\hline $22: 6^{\star *}$ & 1.21 & \pm & 0.23 & 0.81 & \pm 0.19 \\
\hline
\end{tabular}

LDL

\begin{tabular}{|c|c|c|c|c|c|c|}
\hline CE & \multicolumn{3}{|c|}{ control } & \multicolumn{3}{|c|}{ ANGPTL3 LOF } \\
\hline $16: 0$ & 5.28 & \pm & 1.09 & 5.72 & \pm & 0.98 \\
\hline $16: 1^{*}$ & 2.58 & \pm & 0.79 & 5.71 & \pm & 2.57 \\
\hline $18: 1^{*}$ & 10.19 & \pm & 2.24 & 13.92 & \pm & 3.11 \\
\hline $18: 2^{*}$ & 61.22 & \pm & 5.61 & 51.29 & \pm & 7.38 \\
\hline $18: 3$ & 3.31 & \pm & 1.56 & 5.54 & \pm & 4.18 \\
\hline $20: 4$ & 13.96 & \pm & 3.59 & 14.42 & \pm & 2.02 \\
\hline $20: 5$ & 1.85 & \pm & 1.05 & 2.16 & \pm & 1.11 \\
\hline $22: 6^{*}$ & 1.61 & \pm & 0.31 & 1.25 & \pm & 0.24 \\
\hline
\end{tabular}

HDL

\begin{tabular}{crccrcc}
\hline CE & \multicolumn{3}{c}{ control } & \multicolumn{3}{c}{ ANGPTL3 LOF } \\
$16: 0$ & 5.60 & \pm & 0.82 & 6.44 & \pm 1.03 \\
$16: 1$ & 2.70 & \pm & 0.77 & 5.01 & \pm 2.03 \\
$18: 1^{*}$ & 10.56 & \pm & 2.25 & 15.33 & \pm & 3.85 \\
$18: 2^{*}$ & 60.35 & \pm & 5.88 & 50.80 & \pm & 7.85 \\
$18: 3$ & 2.79 & \pm & 0.59 & 3.79 & \pm & 1.69 \\
$20: 4$ & 14.61 & \pm & 4.06 & 15.46 & \pm 2.61 \\
$20: 5$ & 1.75 & \pm & 0.67 & 1.76 & \pm & 0.73 \\
$22: 6$ & 1.63 & \pm & 0.28 & 1.41 & \pm & 0.31
\end{tabular}


Supplementary table 14. Molar percentages of sphingomyelin (SM) species in lipoproteins of control subjects and ANGPTL3 loss-of-function homozygotes (ANGPTL3 LOF). Determined by ESI-MS/MS (mean $\pm \mathrm{SD}, \mathrm{n}=5-10$ ). Statistically significant differences between the groups are marked with asterisks; ${ }^{*} p<0.05,{ }^{* *} \mathrm{p}<0.01,{ }^{* * *} \mathrm{p}<0.001$ (twotailed Student's t-test). All species have a sphingosine 18:1 backbone, except SM 24:2 is likely a mixture of 18:1/24:2 and 18:2/24:1.

VLDL

\begin{tabular}{|c|c|c|c|c|c|c|}
\hline SM & \multicolumn{3}{|c|}{ control } & \multicolumn{3}{|c|}{ ANGPTL3 LOF } \\
\hline 14:0 & 3.39 & \pm & 0.80 & 2.65 & + & 0.53 \\
\hline $15: 0^{*}$ & 2.04 & \pm & 0.36 & 1.41 & \pm & 0.38 \\
\hline $16: 0^{*}$ & 38.91 & \pm & 2.27 & 35.69 & \pm & 2.33 \\
\hline $16: 1^{* \star *}$ & 6.35 & \pm & 0.92 & 4.41 & \pm & 0.73 \\
\hline 17:0 & 0.99 & \pm & 0.22 & 0.86 & \pm & 0.25 \\
\hline 18:0 & 7.58 & \pm & 1.05 & 7.20 & \pm & 1.25 \\
\hline $18: 1^{* * *}$ & 4.24 & \pm & 0.66 & 3.10 & \pm & 0.35 \\
\hline $23: 0$ & 3.51 & \pm & 0.35 & 4.03 & \pm & 0.46 \\
\hline 23:1 & 3.48 & \pm & 0.46 & 3.26 & \pm & 0.70 \\
\hline 24:0 & 4.35 & \pm & 1.06 & 4.51 & \pm & 1.85 \\
\hline $24: 1^{\star * *}$ & 16.54 & \pm & 2.45 & 22.07 & \pm & 1.42 \\
\hline $24: 2$ & 7.87 & \pm & 1.13 & 9.78 & \pm & 2.42 \\
\hline $25: 1$ & 0.75 & \pm & 0.12 & 1.03 & \pm & 0.39 \\
\hline
\end{tabular}

\begin{tabular}{|c|c|c|c|c|c|c|}
\hline SM & & ntrc & & ANGF & TL3 & LOF \\
\hline 14:0 & 3.48 & \pm & 0.86 & 2.87 & \pm & 0.72 \\
\hline 15:0 & 2.04 & \pm & 0.45 & 1.64 & \pm & 0.51 \\
\hline $16: 0$ & 38.19 & \pm & 1.82 & 36.96 & \pm & 2.08 \\
\hline $16: 1^{* *}$ & 5.68 & \pm & 0.66 & 4.27 & \pm & 0.67 \\
\hline 17:0 & 1.13 & \pm & 0.19 & 1.11 & \pm & 0.23 \\
\hline 18:0 & 7.01 & \pm & 0.85 & 7.70 & \pm & 0.77 \\
\hline 18:1 & 3.79 & \pm & 0.67 & 3.29 & \pm & 0.43 \\
\hline 23:0 & 3.94 & \pm & 0.53 & 3.80 & \pm & 0.73 \\
\hline 23:1 & 3.40 & \pm & 0.36 & 2.99 & \pm & 0.34 \\
\hline $24: 0$ & 4.88 & \pm & 0.97 & 4.29 & \pm & 1.09 \\
\hline $24: 1$ & 18.31 & \pm & 2.27 & 20.97 & \pm & 2.28 \\
\hline 24:2 & 7.36 & \pm & 1.94 & 9.09 & \pm & 1.28 \\
\hline $25: 1$ & 0.80 & \pm & 0.19 & 1.02 & \pm & 0.31 \\
\hline
\end{tabular}

\begin{tabular}{|c|c|c|c|c|c|c|}
\hline \multirow{2}{*}{$\begin{array}{l}\text { SM } \\
14: 0\end{array}$} & \multicolumn{3}{|c|}{ control } & \multicolumn{3}{|c|}{ ANGPTL3 LOF } \\
\hline & 2.86 & \pm & 0.81 & 2.20 & \pm & 0.44 \\
\hline 15:0 & 1.68 & \pm & 0.34 & 1.39 & \pm & 0.45 \\
\hline $16: 0^{* *}$ & 32.69 & \pm & 2.33 & 28.25 & \pm & 2.26 \\
\hline $16: 1^{* * *}$ & 6.66 & \pm & 0.72 & 4.42 & \pm & 0.64 \\
\hline $17: 0$ & 1.09 & \pm & 0.23 & 0.93 & \pm & 0.13 \\
\hline 18:0 & 6.63 & \pm & 1.24 & 6.91 & \pm & 0.87 \\
\hline $18: 1^{* *}$ & 4.46 & \pm & 0.87 & 3.34 & \pm & 0.47 \\
\hline 23:0 & 3.92 & \pm & 0.62 & 4.24 & \pm & 0.96 \\
\hline $23: 1$ & 4.00 & \pm & 0.64 & 4.14 & \pm & 0.36 \\
\hline $24: 0$ & 5.08 & \pm & 1.01 & 5.42 & \pm & 1.25 \\
\hline $24: 1^{* * *}$ & 19.94 & \pm & 2.38 & 24.41 & \pm & 1.50 \\
\hline $24: 2$ & 9.99 & \pm & 3.90 & 13.04 & \pm & 2.54 \\
\hline $25: 1$ & 0.98 & \pm & 0.19 & 1.32 & \pm & 0.41 \\
\hline
\end{tabular}


Supplementary table 15. Molar percentages of lyso-phosphatidylcholine (lysoPC) species in lipoproteins of control subjects and ANGPTL3 loss-of-function homozygotes (ANGPTL3 LOF). Determined by ESI-MS/MS (mean \pm SD, $n=5-10$ ). Statistically significant differences between the groups are marked with asterisks; ${ }^{*} p<0.05,{ }^{* *} p<0.01,{ }^{* * *} p<0.001$ (two-tailed Student's t-test).

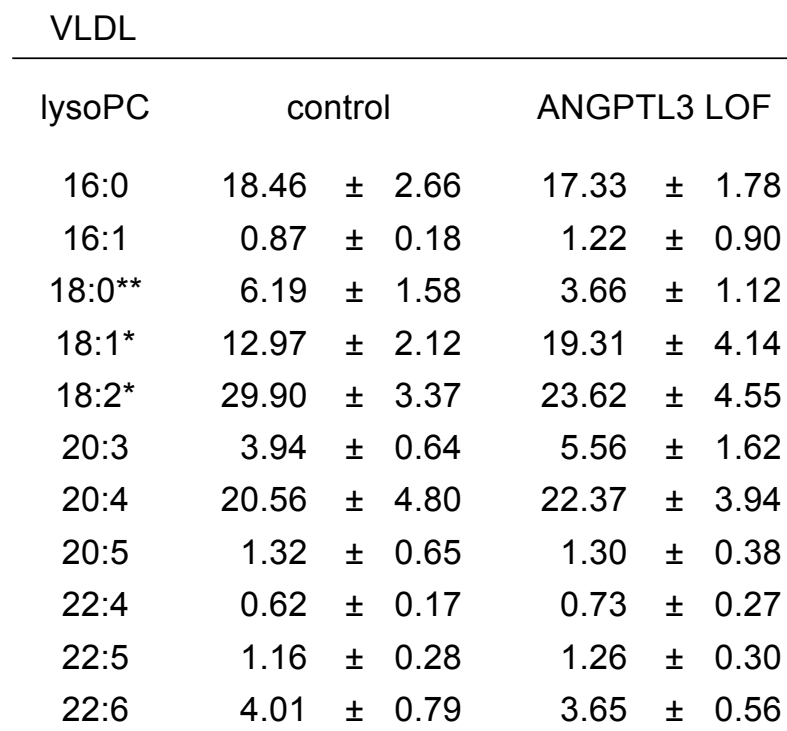

\begin{tabular}{|c|c|c|c|c|c|c|}
\hline lysoPC & \multicolumn{3}{|c|}{ control } & \multicolumn{3}{|c|}{ ANGPTL3 LOF } \\
\hline $16: 0$ & 3.48 & \pm & 0.86 & 2.87 & \pm & 0.72 \\
\hline $16: 1$ & 2.04 & \pm & 0.45 & 1.64 & \pm & 0.51 \\
\hline $18: 0^{*}$ & 38.19 & \pm & 1.82 & 36.96 & \pm & 2.08 \\
\hline $18: 1$ & 5.68 & \pm & 0.66 & 4.27 & \pm & 0.67 \\
\hline $18: 2$ & 1.13 & \pm & 0.19 & 1.11 & \pm & 0.23 \\
\hline $20: 3^{*}$ & 7.01 & \pm & 0.85 & 7.70 & \pm & 0.77 \\
\hline $20: 4^{*}$ & 3.79 & \pm & 0.67 & 3.29 & \pm & 0.43 \\
\hline $20: 5$ & 3.94 & \pm & 0.53 & 3.80 & \pm & 0.73 \\
\hline $22: 4^{* *}$ & 3.40 & \pm & 0.36 & 2.99 & \pm & 0.34 \\
\hline $22: 5^{\star}$ & 4.88 & \pm & 0.97 & 4.29 & \pm & 1.09 \\
\hline $22: 6$ & 18.31 & \pm & 2.27 & 20.97 & \pm & 2.28 \\
\hline
\end{tabular}

\begin{tabular}{|c|c|c|c|c|c|}
\hline lysoPC & \multicolumn{3}{|c|}{ control } & \multicolumn{2}{|c|}{ ANGPTL3 LOF } \\
\hline $16: 0^{*}$ & 23.59 & \pm & 1.48 & 18.04 & \pm 4.49 \\
\hline $16: 1$ & 0.85 & \pm & 0.09 & 1.77 & \pm 1.20 \\
\hline $18: 0^{\star * *}$ & 9.35 & \pm & 1.60 & 5.31 & \pm 1.52 \\
\hline 18:1 & 14.04 & \pm & 2.20 & 16.26 & \pm 3.25 \\
\hline $18: 2$ & 25.19 & \pm & 3.97 & 22.12 & \pm 4.53 \\
\hline $20: 3^{*}$ & 3.87 & \pm & 0.94 & 5.13 & \pm 0.89 \\
\hline $20: 4^{*}$ & 16.61 & \pm & 3.87 & 23.16 & \pm 5.06 \\
\hline $20: 5$ & 1.02 & \pm & 0.56 & 1.24 & \pm 0.38 \\
\hline $22: 4^{* *}$ & 0.48 & \pm & 0.12 & 0.93 & \pm 0.18 \\
\hline $22: 5^{\star}$ & 1.09 & \pm & 0.34 & 1.63 & \pm 0.40 \\
\hline 22:6 & 3.92 & \pm & 0.50 & 4.41 & \pm 0.76 \\
\hline
\end{tabular}


Supplementary table 16. Molar percentages of phosphatidylcholine (PC) species in lipoproteins of control subjects and ANGPTL3 loss-of-function homozygotes (ANGPTL3 LOF). Determined by ESI-MS/MS (mean \pm SD, $n=5-10$ ). Statistically significant differences between the groups are marked with asterisks; ${ }^{*} p<0.05,{ }^{* *} p<0.01,{ }^{* \star *} p<0.001$ (two-tailed Student's t-test). a=alkyl-acyl species.

VLDL

\begin{tabular}{|c|c|c|c|c|c|c|}
\hline PC & \multicolumn{3}{|c|}{ control } & \multicolumn{3}{|c|}{ ANGPTL3 LOF } \\
\hline $32: 0^{*}$ & 0.89 & \pm & 0.10 & 1.33 & \pm & 0.26 \\
\hline $32: 1$ & 0.81 & \pm & 0.35 & 2.06 & \pm & 1.48 \\
\hline $34: 1$ & 14.65 & \pm & 1.90 & 18.12 & \pm & 3.15 \\
\hline $34: 1 a^{* \star *}$ & 0.66 & \pm & 0.14 & 1.15 & \pm & 0.16 \\
\hline $34: 2$ & 27.83 & \pm & 3.49 & 21.70 & \pm & 6.31 \\
\hline $34: 2 a$ & 0.61 & \pm & 0.21 & 0.67 & \pm & 0.19 \\
\hline $34: 3^{*}$ & 0.92 & \pm & 0.95 & 0.00 & \pm & 0.00 \\
\hline $36: 1$ & 2.02 & \pm & 0.43 & 2.78 & \pm & 2.06 \\
\hline $36: 1 a$ & 0.39 & \pm & 0.09 & 0.49 & \pm & 0.09 \\
\hline $36: 2$ & 13.38 & \pm & 2.50 & 11.24 & \pm & 2.27 \\
\hline $36: 2 a$ & 0.75 & \pm & 0.19 & 0.67 & \pm & 0.10 \\
\hline $36: 3$ & 7.31 & \pm & 1.02 & 7.75 & \pm & 0.93 \\
\hline $36: 3 a^{*}$ & 0.44 & \pm & 0.11 & 0.76 & \pm & 0.23 \\
\hline $36: 4$ & 10.77 & \pm & 2.67 & 9.95 & \pm & 1.81 \\
\hline $36: 4 a^{* *}$ & 1.04 & \pm & 0.22 & 1.96 & \pm & 0.46 \\
\hline $36: 5$ & 1.24 & \pm & 0.54 & 1.20 & \pm & 0.34 \\
\hline $38: 3$ & 1.79 & \pm & 0.55 & 2.27 & \pm & 0.96 \\
\hline $38: 4$ & 5.27 & \pm & 1.68 & 5.42 & \pm & 1.37 \\
\hline $38: 4 a$ & 0.74 & \pm & 0.14 & 0.83 & \pm & 0.09 \\
\hline $38: 5$ & 2.04 & \pm & 0.64 & 2.51 & \pm & 0.60 \\
\hline $38: 5 a^{* * *}$ & 0.82 & \pm & 0.18 & 1.71 & \pm & 0.16 \\
\hline $38: 6$ & 3.87 & \pm & 1.03 & 3.46 & \pm & 0.59 \\
\hline $38: 6 a^{* * *}$ & 0.27 & \pm & 0.07 & 0.44 & \pm & 0.06 \\
\hline $40: 5$ & 0.31 & \pm & 0.10 & 0.34 & \pm & 0.18 \\
\hline $40: 6$ & 1.17 & \pm & 0.35 & 1.20 & \pm & 0.59 \\
\hline
\end{tabular}

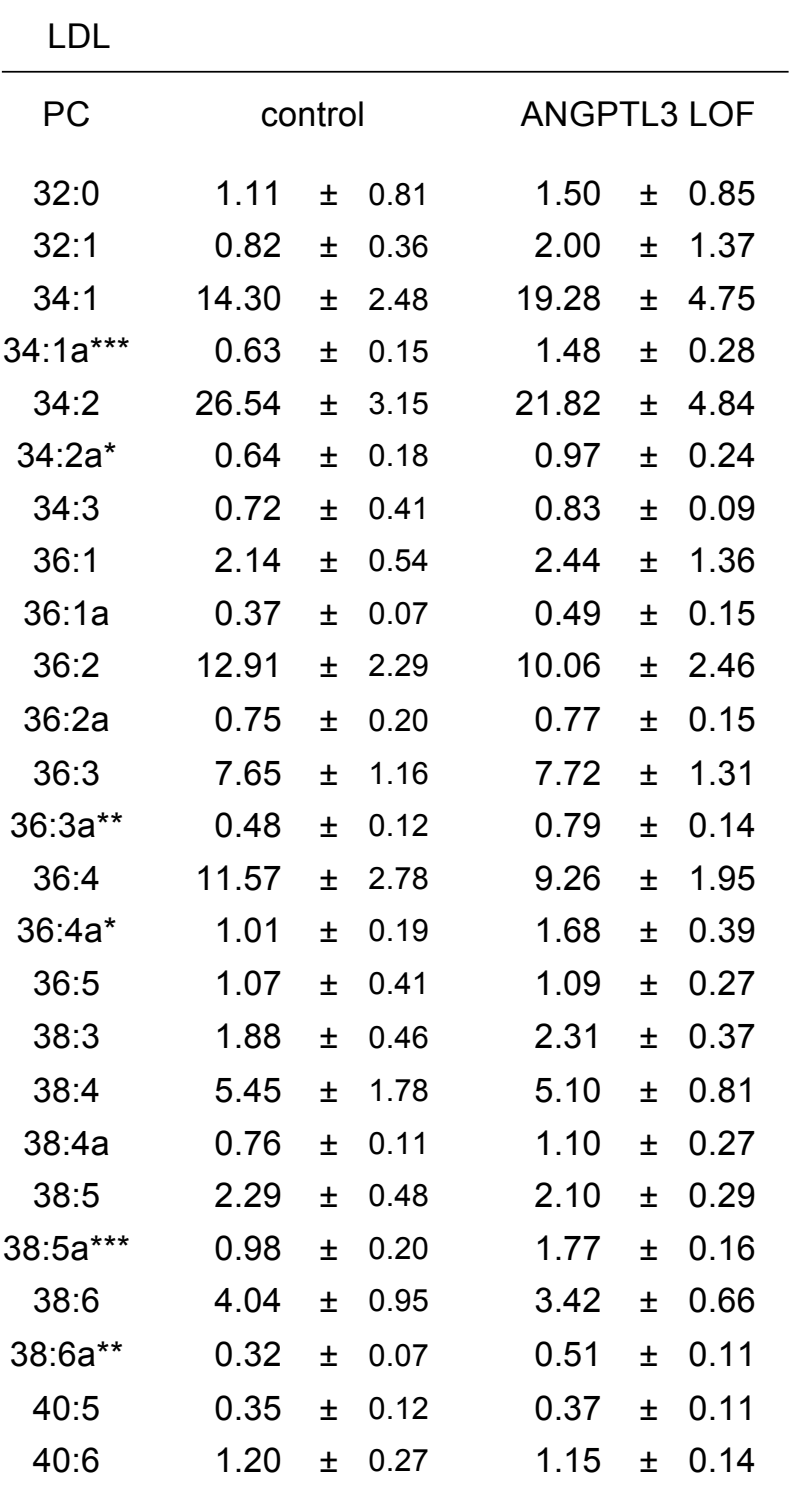

\begin{tabular}{|c|c|c|c|c|c|c|}
\hline \multirow{2}{*}{$\begin{array}{c}\mathrm{PC} \\
32: 0^{* *}\end{array}$} & \multicolumn{3}{|c|}{ control } & \multicolumn{3}{|c|}{ ANGPTL3 LOF } \\
\hline & 0.53 & \pm & 0.06 & 0.82 & \pm & 0.13 \\
\hline $32: 1$ & 0.81 & \pm & 0.30 & 2.02 & \pm & 1.31 \\
\hline $34: 1^{*}$ & 11.77 & \pm & 2.52 & 16.00 & \pm & 3.23 \\
\hline $34: 1 a^{* *}$ & 0.49 & \pm & 0.08 & 1.11 & \pm & 0.24 \\
\hline $34: 2$ & 25.97 & \pm & 3.04 & 20.70 & \pm & 5.08 \\
\hline $34: 2 a^{*}$ & 0.57 & \pm & 0.15 & 0.91 & \pm & 0.23 \\
\hline $34: 3^{*}$ & 0.77 & \pm & 0.17 & 1.05 & \pm & 0.22 \\
\hline $36: 1$ & 1.61 & \pm & 0.54 & 2.24 & \pm & 1.29 \\
\hline $36: 1 a$ & 0.35 & \pm & 0.07 & 0.46 & \pm & 0.16 \\
\hline $36: 2^{*}$ & 12.20 & \pm & 1.95 & 9.45 & \pm & 2.15 \\
\hline $36: 2 a$ & 0.72 & \pm & 0.17 & 0.76 & \pm & 0.07 \\
\hline $36: 3$ & 8.42 & \pm & 1.20 & 7.80 & \pm & 1.00 \\
\hline $36: 3 a^{* *}$ & 0.52 & \pm & 0.11 & 0.84 & \pm & 0.14 \\
\hline $36: 4$ & 13.40 & \pm & 2.65 & 11.33 & \pm & 2.40 \\
\hline $36: 4 a^{*}$ & 1.09 & \pm & 0.25 & 1.89 & \pm & 0.51 \\
\hline $36: 5$ & 1.34 & \pm & 0.55 & 1.38 & \pm & 0.28 \\
\hline $38: 3$ & 1.60 & \pm & 0.55 & 1.90 & \pm & 0.36 \\
\hline $38: 4$ & 5.71 & \pm & 1.50 & 5.59 & \pm & 0.71 \\
\hline $38: 4 a^{* *}$ & 0.85 & \pm & 0.17 & 1.32 & \pm & 0.28 \\
\hline $38: 5$ & 2.90 & \pm & 0.55 & 3.07 & \pm & 0.62 \\
\hline $38: 5 a^{* \star *}$ & 1.30 & \pm & 0.28 & 2.61 & \pm & 0.35 \\
\hline $38: 6$ & 4.92 & \pm & 0.99 & 4.26 & \pm & 0.61 \\
\hline $38: 6 a^{* * *}$ & 0.46 & \pm & 0.11 & 0.75 & \pm & 0.07 \\
\hline $40: 5$ & 0.36 & \pm & 0.09 & 0.45 & \pm & 0.17 \\
\hline $40: 6$ & 1.33 & \pm & 0.28 & 1.30 & \pm & 0.41 \\
\hline
\end{tabular}


*Declaration of Interest Statement
Click here to download Declaration

Click here to download Declaration of Interest Statement: Conflict of interest.docx

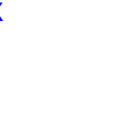

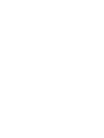

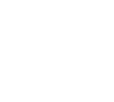

$\sqrt{10}$

$\sqrt{10}$

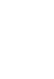

$\sqrt{2}$

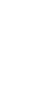

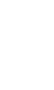

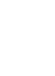
(1)

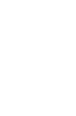
(2) (n) . 


\section{Credit author statement}

Hanna Ruhanen: Investigation; Methodology; Writing - original draft

Nidhina Haridas: Conceptualization; Methodology; Supervision; Writing original draft; Writing - review \& editing

Ilenia MInicocci: Resources (human subjects); Writing - review \& editing

Juuso H. Taskinen: Investigation; Writing - review \& editing

Francesco Palmas: Investigation; Methodology

Alessia di Costanzo: Resources (human subjects); Writing - review \& editing Laura D’Erasmo: Resources (human subjects); Writing - review \& editing Jari Metso: Investigation; Methodology

Jennimari Partanen: Investigation

Jesmond Dalli: Conceptualization; Data curation; Funding acquisition; Project administration; Writing - review \& editing

You Zhou: Investigation (RNAseq data analysis); Writing - review \& editing

Marcello Arca: Conceptualization; Funding acquisition; Resources (human subjects); Writing - review \& editing

Matti Jauhiainen: Conceptualization; Funding acquisition; Methodology; Supervision; Writing - review \& editing

Reijo Käkelä: Conceptualization; Methodology; Project administration; Supervision; Writing - review \& editing

Vesa M. Olkkonen: Conceptualization; Funding acquisition; Project administration; Supervision; Writing - review \& editing 